EFI-03-44

WIS/26/03-OCT-DPP

hep-th/0310197

\title{
Little String Theory and Heterotic/Type II Duality
}

\author{
Ofer Aharony ${ }^{a}$, Bartomeu Fiol $^{a}$, David Kutasov ${ }^{b}$ and David A. Sahakyan ${ }^{b}$ \\ ${ }^{a}$ Department of Particle Physics, Weizmann Institute of Science, Rehovot 76100, Israel \\ Ofer.Aharony, fiol@weizmann.ac.il \\ ${ }^{b}$ EFI and Department of Physics, University of Chicago \\ 5640 S. Ellis Av., Chicago, IL 60637, USA \\ kutasov, sahakian@theory.uchicago.edu
}

Little String Theory (LST) is a still somewhat mysterious theory that describes the dynamics near a certain class of time-like singularities in string theory. In this paper we discuss the topological version of LST, which describes topological strings near these singularities. For $5+1$ dimensional LSTs with sixteen supercharges, the topological version may be described holographically in terms of the $\mathcal{N}=4$ topological string (or the $\mathcal{N}=2$ string) on the transverse part of the near-horizon geometry of $N S 5$-branes. We show that this topological string can be used to efficiently compute the half-BPS $F^{4}$ terms in the lowenergy effective action of the LST. Using the strong-weak coupling string duality relating type IIA strings on $K 3$ and heterotic strings on $T^{4}$, the same terms may also be computed in the heterotic string near a point of enhanced gauge symmetry. We study the $F^{4}$ terms in the heterotic string and in the LST, and show that they have the same structure, and that they agree in the cases for which we compute both of them. We also clarify some additional issues, such as the definition and role of normalizable modes in holographic linear dilaton backgrounds, the precise identifications of vertex operators in these backgrounds with states and operators in the supersymmetric Yang-Mills theory that arises in the low energy limit of LST, and the normalization of two-point functions. 


\section{Introduction}

One of the more mysterious outcomes of the recent progress in the understanding of non-perturbative aspects of string theory is the discovery of theories which are non-local (and have some stringy aspects) but are decoupled from gravity. These theories are known as Little String Theories (LSTs) (for reviews see [1,2]); they appear in backgrounds of string theory which contain singularities and/or NS5-branes. In these cases interesting dynamics near the singularity (or brane) remains even in the decoupling limit $g_{s} \rightarrow 0$. This dynamics is captured by the LST associated with the singularity.

The best description that we have for these theories [3] is via an asymptotically linear dilaton background of string theory which is holographically dual to them 1 . This description tells us what are the observables of the LST, and some of its properties (such as the thermodynamic behavior, which at high energy densities resembles that of free string theories, with important differences [5]). It also allows for the computation of some correlation functions in these theories (at least when we go out on their moduli space [6]7]). However, we still lack a direct definition of these theories.

In many supersymmetric compactifications of string theory, there is a sector of the theory which is protected by supersymmetry (the analog of the chiral sector in $d=4, \mathcal{N}=1$ supersymmetric gauge theories), and which is captured by a topological version of the full string theory. In the case of type II string theories compactified on Calabi-Yau three-folds, this topological string theory is the $\mathcal{N}=2$ topological string [8], while for type II string theories compactified on $K 3$ surfaces it is the $\mathcal{N}=4$ topological string, which is equivalent to the $\mathcal{N}=2$ string [9] (see also [10]). Since the construction of a Little String Theory involves taking some limit of a supersymmetric type II compactification, it is natural to suggest that taking the same limit in the topological string theory will lead to a topological version of Little String Theory. Such a version may be easier to understand than the full LST, and it may be easier to find a direct definition for it (the holographic description of these theories is just the topological string version of the holographic description of the full LSTs). In four dimensional LSTs, some progress in this direction has been made, particularly for the case of the conifold [11-15]. The main motivation for this work is to obtain a better understanding of more general singularities of Calabi-Yau as well as $K 3$ surfaces.

1 In some cases there is also a DLCQ description [4], but this requires taking a large $N$ limit which is difficult to control. The holographic description does not require taking large $N$ limits. 
In this paper we focus on the most symmetric LSTs, which are $5+1$ dimensional theories with sixteen supercharges $(\mathcal{N}=(1,1)$ supersymmetry in six dimensions). These theories arise from decoupling limits of type IIA strings on ALE spaces (non-compact $K 3$ manifolds that arise by blowing up the geometry in the vicinity of ADE singularities of compact $K 3$ surfaces), or from decoupling limits of type IIB $N S 5$-branes in flat space. The holographic description of these theories is given by string theory on the near-horizon geometry of NS5-branes, the CHS background [16]. The discussion above suggests that the $\mathcal{N}=2$ string on the CHS background is holographically dual to a topological version of the corresponding LST. In this paper we investigate this suggestion.

Before taking any decoupling limits, the topological string computes various amplitudes which are protected by supersymmetry in the full type II string theory [9]. These correspond to the coefficients of specific terms in the low-energy effective action of the theory. It is interesting to ask what do correlation functions in the topological version of LST compute. In general, LSTs are known to have operators which are defined off-shell, and the physical observables are Green's functions of these operators. This is different from critical string theory, in which the observables are on-shell S-matrix elements. The discussion above suggests that there should be some sub-class of the correlation functions of the off-shell observables of LST which is topological in nature, and which is computed by the topological LST. One way to derive this sub-class is by taking a limit of the topological observables of the full type II string theory, but this is complicated by the difference between the observables in the two types of theories. Another way to derive this sub-class is to follow the terms in the effective action of the type II string theory which are protected by supersymmetry to the LST limit, and to find which observables in the topological LST compute these terms. This is the route that we will follow in this paper.

The particular term in the low-energy effective action that we will discuss has the form $t_{8} F^{4}$, where $F$ is an Abelian gauge field in the low-energy theory and $t_{8}$ is a specific constant tensor which governs the contraction of the indices of the four gauge fields. In weakly coupled type II string theory, the field strength in question corresponds to a RamondRamond (RR) gauge field. The coefficient of this $F^{4}$ term is believed to be protected from quantum corrections by supersymmetry, and to be given exactly by the tree level contribution. We will show that it corresponds to an observable in the topological string theory, and use this to compute it.

We will leave the analysis of other topological amplitudes in LST to future work. Such amplitudes are also of interest; for example, it is known that higher-loop contributions to 
the partition sum of the topological string theory are related to the coefficients of certain $R^{4} F^{4 g-4}$ terms in the effective action of the type II string theory [9].

Type IIA string theory on $K 3$ is believed to be dual to the heterotic string on $T^{4}$, and the coefficient of the $t_{8} F^{4}$ term may also be computed using the heterotic theory. In the heterotic string, this term receives contributions only at one-loop, and it can be easily computed. The result simplifies significantly in the LST decoupling limit, where it is given by a one-loop computation in the low-energy gauge theory.

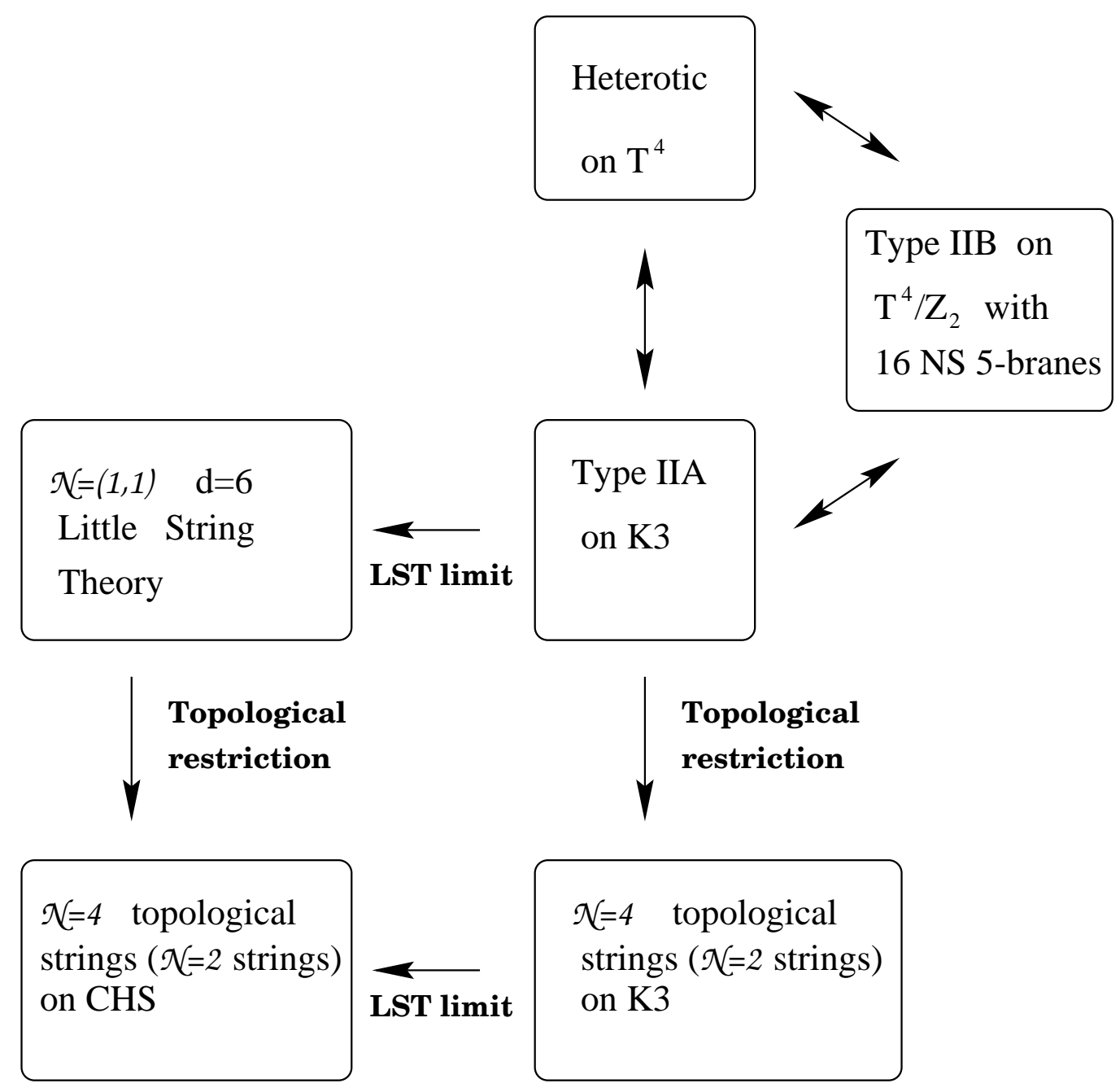

Fig. 1: A schematic diagram of the theories discussed in this paper. The three theories on the top-right corner are related by T-dualities and S-dualities. The different routes towards the bottom-left corner are commutative.

Heterotic/type II duality thus leads to a non-trivial prediction: a tree level four-point function in LST should be equal to a one-loop amplitude in the low-energy field theory. As 
we review below, the two calculations are valid in different regions in moduli space, and the only reasons to expect them to agree are (1) the non-renormalization of these terms, and (2) heterotic/type II duality. We will show by an explicit calculation of both sides that they indeed agree. One can view this as a new non-trivial check of heterotic/type II duality and of the non-renormalization of the $t_{8} F^{4}$ term. Since these two circles of ideas are rather well established, we use these calculations to develop tools for studying LST in general, and particularly its topological sector.

One of our main motivations for studying these theories is the hope that an alternative description of LST can be obtained by using an open-closed string large $N$ duality. There are some indications that this should be possible. The first example of a holographic duality, the equivalence of certain large $N$ matrix models in one or less dimensions in a double scaling limit with $c \leq 1$ conformal field theories (CFTs) coupled to worldsheet gravity (or string theory in $D \leq 2$ dimensions), is now understood as an open-closed string duality, with the open strings living on unstable $D 0$-branes localized in the Liouville direction 1725]. These backgrounds of string theory can be thought of as simple examples of LST [3,26], with the Liouville direction playing the role of the radial direction away from the singularity.

Thus, it is natural to expect more generally, that an alternative description of LST can be obtained by studying D-branes localized in the vicinity of the singularity (where, as we will review below, the effective string coupling is largest). A natural first step in constructing an open string dual of the full LST is to find one that is dual to the topological sector of the theory. Something like this is known to exist for the case of the conifold, where the topological LST is dual to a topological open string theory describing the dynamics on $N \rightarrow \infty$ D-branes localized near the conifold singularity 11, 12,13. We would like to find the analog for the case of ALE spaces. We will not discuss open-closed string duality in this paper (except for a few comments in the discussion), but we hope that our results will be useful for constructing an open string dual for six dimensional topological LST, and perhaps eventually also for the full theory.

We begin in section 2 by reviewing the known results about $t_{8} F^{4}$ terms in field theories with sixteen supercharges and in toroidal compactifications of the heterotic string. In section 3 we review the duality between the heterotic string on $T^{4}$, the type IIA string on $K 3$, and type IIB backgrounds with NS5-branes, and the implications of this duality for the $t_{8} F^{4}$ terms. In section 4 we formulate type II string theory in the near-horizon limit of ALE singularities (or coincident NS5-branes), and discuss in detail its worldsheet 
properties. We also comment on the analogy between our discussion of the ALE case and previous discussions of strings on the conifold. In section 5 we use the type II and $\mathcal{N}=2$ string theories on the (deformed) CHS background to compute correlation functions of the vertex operators which are relevant for the $t_{8} F^{4}$ terms in the low-energy effective action. In section 6 we compare our type II and $\mathcal{N}=2$ string results with the expectations from the heterotic (or low-energy field theory) analysis. We end in section 7 with a summary of our results and a discussion of possible future directions. Four appendices contain useful technical results.

\section{2. $F^{4}$ terms in theories with sixteen supercharges}

In this section we review the structure of $F^{4}$ terms in the effective actions of theories with sixteen supercharges, both in field theory and in string theory. We review the arguments for the one-loop exactness of these terms. For the string theory case, we focus in this section on the heterotic string, since the relation with the field theory limit there is most straightforward. In the next section we discuss the appearance of the $F^{4}$ terms in different string duals of the background considered here.

\subsection{Field theory}

In super Yang-Mills perturbation theory, there is a one-loop contribution to $F^{4}$ terms in the low-energy effective action, which is given by the diagrams with four external gauge bosons in figure 2. As reviewed below, for the $d=4 \mathcal{N}=4$ supersymmetric Yang-Mills (SYM) theory the one-loop contribution gives the exact result, both perturbatively and non-perturbatively. On the other hand, it is known [27] that for theories with sixteen supercharges in $d=3$ the $F^{4}$ terms receive instanton corrections.

The non-renormalization of $F^{4}$ terms in $d=4, \mathcal{N}=4$ SYM has been discussed by various authors [27-30]. On general grounds, the terms for which we expect nonrenormalization would be integrals over half of $\mathcal{N}=4$ superspace, if such a formulation existed. Consider an $S U(2)$ gauge theory spontaneously broken to $U(1)$. In such a case the low-energy effective action involves only a $U(1) \mathcal{N}=4$ vector multiplet. When we view the $\mathcal{N}=4$ theory as an $\mathcal{N}=2$ theory, the $U(1)$ gauge field strength $F_{\mu \nu}$ appears as a component of the $\mathcal{N}=2 U(1)$ vector multiplet $\Psi$. We consider the $F^{4}$ terms in the region of moduli space where only the scalar in the $\mathcal{N}=2$ vector multiplet has a vacuum 

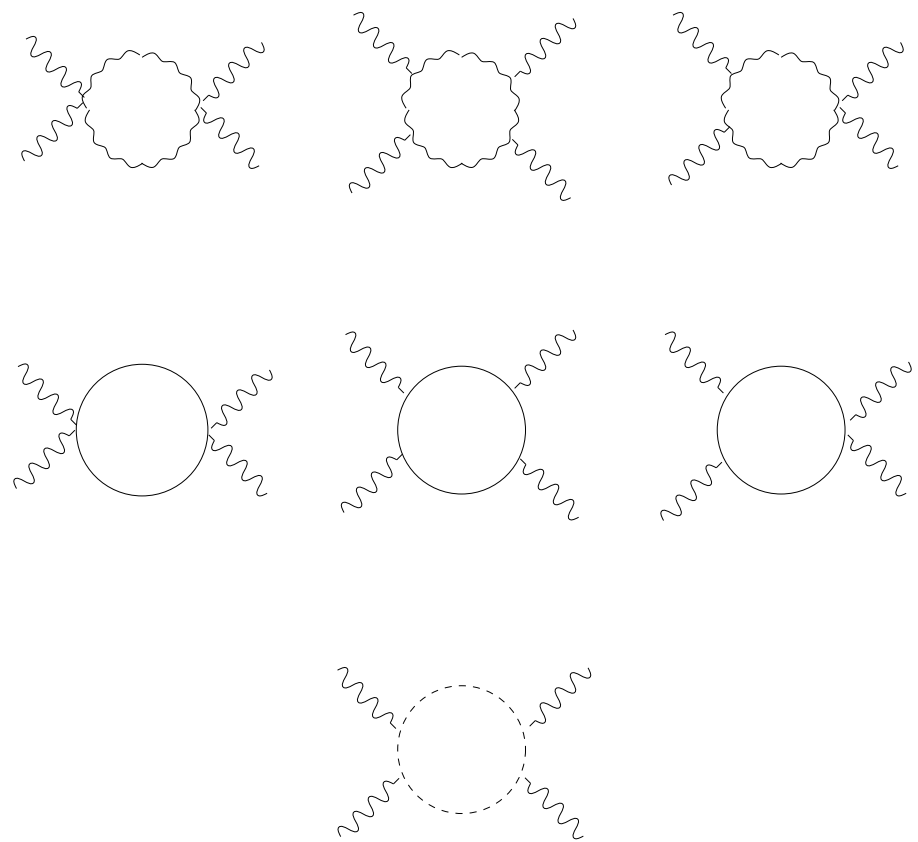

Fig. 2: Diagrams contributing to $F^{4}$ terms in SYM theories. Wiggly lines denote gauge fields, solid lines are scalar fields and dashed lines are fermions.

expectation value $(\mathrm{VEV})$ 2. In $\mathcal{N}=2$ superspace language, the most general such term in the low-energy effective action can be written as

$$
\int d^{8} \theta \mathcal{H}\left(\Psi, \Psi^{\dagger}, \tau, \tau^{\dagger}\right),
$$

where $\tau$ is the complexified coupling constant. Invoking the scale invariance and the $U(1)_{R}$ symmetry of the theory, one can argue that for given $\tau, \mathcal{H}$ is uniquely fixed to be

$$
\mathcal{H} \sim \ln \left(\frac{\Psi}{\Lambda}\right) \ln \left(\frac{\Psi^{\dagger}}{\Lambda}\right)
$$

where $\Lambda$ is a fake scale, that does not survive integration over superspace. Furthermore, by promoting $\tau$ to a background superfield, it also follows from scale invariance and $U(1)_{R}$ symmetry that $\mathcal{H}$ can not depend on $\tau$ at all, so the coefficient in (2.2) can be computed at one-loop, and there are neither perturbative nor non-perturbative corrections to the one-loop result.

It is easy to generalize this to arbitrary gauge groups (broken to their Abelian subgroup by a VEV for the scalar in the vector multiplet) 333 35 , where $\vec{\Psi}$ belongs to the Cartan

2 The more general case was discussed in 29, 30,31] and reviewed in [32]. 
subalgebra and $\mathcal{H}$ is given by a sum over the positive roots of the gauge group

$$
\mathcal{H} \sim \sum_{\vec{\alpha}>0} \ln \left(\frac{\vec{\Psi} \cdot \vec{\alpha}}{\Lambda}\right) \ln \left(\frac{\overrightarrow{\Psi^{\dagger}} \cdot \vec{\alpha}}{\Lambda}\right)
$$

After integration over superspace this gives rise to $F^{4}$ terms. Similar terms appear at oneloop in any dimension (in theories with sixteen supercharges), and in the $d$-dimensional maximally supersymmetric SYM theory they are given by

$$
\mathcal{L}_{e f f} \sim \sum_{\vec{\alpha}>0} \frac{(\vec{F} \cdot \vec{\alpha})^{4}}{M_{W}^{8-d}(\vec{\alpha})}
$$

where $M_{W}(\vec{\alpha})$ is the mass of the W-boson associated with the root $\vec{\alpha}$ (at a particular point in the moduli space). The structure of the space-time indices in (2.4) will be described below.

As mentioned above, for $d=4$ equation (2.4) is exact, while for $d=3$ it receives instanton corrections. For $d>4$ the gauge theory is non-renormalizable, and one has to embed it in a consistent theory, such as string theory, in order to discuss higher loop and non-perturbative corrections.

\subsection{String theory}

Next, we turn to $F^{4}$ terms in string theories with sixteen supercharges. For convenience, we will start by considering the field strength $F_{\mu \nu}$ to be that of the $S O(32)$ heterotic string theory in ten dimensions. The interesting term is of the form $t_{8} F^{4}$, where $t_{8}$ is a constant tensor defined below. In ten dimensions, the $t_{8} \operatorname{tr}\left(F^{4}\right)$ term is absent at tree level3, but it receives a one-loop contribution [36-39]. Since it is related by supersymmetry to the anomaly-canceling term $B F^{4}$, the one-loop result is expected to be exact, both perturbatively and non-perturbatively. The precise result for the amplitude in ten dimensions is

$$
\frac{1}{2^{8} \pi^{5} 4 ! \alpha^{\prime}} t_{8}^{\mu \nu \sigma \rho \alpha \beta \gamma \delta} \operatorname{Tr}_{v}\left(F_{\mu \nu} F_{\sigma \rho} F_{\alpha \beta} F_{\gamma \delta}\right)
$$

3 There is a tree-level $t_{8} \operatorname{tr}\left(F^{2}\right) \operatorname{tr}\left(F^{2}\right)$ term, but this term will not be relevant in the limit we will be interested in. 
where the trace is in the vector representation and the tensor $t_{8}$ is defined as follows :

$$
\begin{aligned}
t_{8}^{\mu \nu \sigma \rho \alpha \beta \gamma \delta \equiv-} \equiv & \left(\delta^{\mu \sigma} \delta^{\nu \rho}-\delta^{\mu \rho} \delta^{\nu \sigma}\right)\left(\delta^{\alpha \gamma} \delta^{\beta \delta}-\delta^{\alpha \delta} \delta^{\beta \gamma}\right)+ \\
& \left(\delta^{\sigma \alpha} \delta^{\rho \beta}-\delta^{\sigma \beta} \delta^{\rho \alpha}\right)\left(\delta^{\gamma \mu} \delta^{\delta \nu}-\delta^{\gamma \nu} \delta^{\delta \mu}\right)+ \\
& \left.\left(\delta^{\mu \alpha} \delta^{\nu \beta}-\delta^{\mu \beta} \delta^{\nu \alpha}\right)\left(\delta^{\sigma \gamma} \delta^{\rho \delta}-\delta^{\sigma \delta} \delta^{\rho \gamma}\right)\right\}+ \\
& \frac{1}{2}\left\{\delta^{\nu \sigma} \delta^{\rho \alpha} \delta^{\beta \gamma} \delta^{\delta \mu}+\delta^{\nu \alpha} \delta^{\beta \sigma} \delta^{\rho \gamma} \delta^{\delta \mu}+\delta^{\nu \alpha} \delta^{\beta \gamma} \delta^{\delta \sigma} \delta^{\rho \mu}+\text { antisymmetrization }\right\} .
\end{aligned}
$$

When we compactify the heterotic string on $T^{4}$, the structure of the $t_{8} F^{4}$ terms becomes more interesting. Now, at generic points in moduli space the gauge group is $U(1)^{24}$. We focus on the $t_{8} F^{4}$ term involving only the sixteen gauge bosons coming from the Cartan torus of $S O(32)$. The coefficient of this term is no longer fixed by anomaly considerations, and it depends on the Narain moduli. At the same time, there are several arguments (reviewed, for example, in [40]) that support the claim that this term does not depend on the heterotic string coupling $g_{h}$, i.e. it is one-loop exact. Perturbative corrections in $g_{h}$ can be shown to be absent, as in the ten dimensional case (see e.g. [41]); this was explicitly verified at two-loop order in [42]. Non-perturbatively, the only identifiable BPS instanton is the heterotic fivebrane, but there are no six-cycles in $T^{4}$ which the Euclidean fivebrane can wrap. Finally, the independence from the string coupling is suggested by the decoupling between the gravitational multiplet (to which the dilaton belongs) and the vector multiplets, as seen in the factorization of the moduli space.

The computation of the one-loop contribution to the effective Lagrangian near singularities in the case at hand is very similar to the one performed for the conifold in [43]. The term in the effective Lagrangian arising from the one-loop computation is

$$
\mathcal{L}_{e f f}=l_{h}^{2} t_{8} F^{I} F^{J} F^{K} F^{L} \int_{\mathcal{F}} \frac{d^{2} \tau}{\tau_{2}^{2}} \sum_{\left(p_{L}, p_{R}\right) \in \Gamma_{4,20}} p_{R}^{I} p_{R}^{J} p_{R}^{K} p_{R}^{L} \tau_{2}^{2} \frac{q^{\frac{1}{2} p_{L}^{2}} \bar{q}^{\frac{1}{2} p_{R}^{2}}}{\bar{\eta}(\bar{\tau})^{24}}
$$

where we suppressed the space-time indices of $t_{8}$ and of the gauge fields, which run over $0, \ldots, 5 . \mathcal{F}$ is the fundamental domain of the moduli space of complex structures of the (worldsheet) torus, $l_{h}=1 / M_{h}$ is the heterotic string length, and the sum runs over the even, self-dual lattice $\Gamma_{4,20}$ corresponding to a particular value of the Narain moduli. The contribution $\tau_{2}^{2}$ comes from the zero modes on $T^{4}$, and the $1 / \bar{\eta}(\bar{\tau})^{24}$ is the contribution of 
the right-moving bosonic lattice. To describe the points of enhanced gauge symmetry in the moduli space, we start by recalling the mass formula for perturbative BPS states [44]:

$$
\frac{1}{4} \alpha^{\prime} M^{2}=\frac{1}{2} p_{L}^{2}=\frac{1}{2} p_{R}^{2}+\left(N_{R}-1\right)
$$

The sixteen gauge bosons of the Cartan subalgebra have $p_{L}=p_{R}=0$ and $N_{R}=1$, and are massless everywhere in moduli space. At the origin of moduli space there are additional massless states with $N_{R}=0$ and $p_{L}^{2}=0, p_{R}^{2}=2$. These are W-bosons corresponding to roots of $S O(32)$. At generic points in the moduli space of $\Gamma_{4,20}$ they are massive. We will be interested in the behavior near points of enhanced ADE gauge symmetry, where some or all of them are light. Near such points, the expression (2.7) simplifies enormously. The leading contribution comes from the light W-bosons, and is dominated by $\tau_{2} \rightarrow \infty$ :

$$
\int_{\mathcal{F}} d^{2} \tau e^{-\pi \tau_{2} \alpha^{\prime} M_{W}^{2}}=\frac{1}{\pi \alpha^{\prime} M_{W}^{2}}\left(1+\mathcal{O}\left(\alpha^{\prime} M_{W}^{2}\right)\right)
$$

where $M_{W}$ is the mass of the light $W$ boson.

All in all, near a point of enhanced gauge symmetry, the effective Lagrangian (2.7) reduces to

$$
\mathcal{L}_{e f f} \sim \sum_{\vec{\alpha}>0} \frac{t_{8}(\vec{F} \cdot \vec{\alpha})^{4}}{M_{W}^{2}(\vec{\alpha})}\left(1+\mathcal{O}\left(M_{W}^{2} / M_{h}^{2}\right)\right)
$$

where the sum runs over the roots of the gauge group corresponding to the light gauge bosons at a particular point in moduli space. As expected, this is the same as the one-loop result in the low-energy field theory described above. It is easy to show that the string calculation reduces in this limit to the field theory one-loop calculation reviewed in $\S 2.1$.

\section{Heterotic/type II duality}

In this section we review the duality between the heterotic string on $T^{4}$, the type IIA string on $K 3$, and configurations of $N S 5$-branes in type IIB string theory, paying special attention to the origin of the $t_{8} F^{4}$ terms in these backgrounds. We will focus on the behavior near points in moduli space where the gauge symmetry is enhanced. 


\subsection{Duality for compact spaces}

There is strong evidence for a strong-weak coupling duality between type IIA string theory on $K 3$ and heterotic string theory on $T^{4}$ [45]. In particular, their six dimensional $\mathcal{N}=(1,1)$ supersymmetric effective actions can be identified by a change of variables that implies the following relations between the six dimensional string couplings and string scales :

$$
l_{h}=g_{I I} l_{I I}, \quad g_{h}=1 / g_{I I}
$$

The massless content of type IIA string theory on $K 3$ at generic points in moduli space is one $\mathcal{N}=(1,1)$ graviton multiplet and twenty $\mathcal{N}=(1,1)$ vector multiplets. Since the $\mathcal{N}=(1,1)$ graviton multiplet contains four graviphotons, at generic points in moduli space the gauge group is $U(1)^{24}$, as on the heterotic side. Since all the gauge bosons are in the Ramond-Ramond (RR) sector, no perturbative string states are charged under them, only $D$-branes.

Twenty two of the twenty four gauge bosons come from reductions of the type IIA RR three-form potential on two-cycles of the $K 3$. Of these twenty two, three correspond to self-dual forms and nineteen to anti-self-dual forms. Sixteen of these nineteen gauge bosons map to the sixteen gauge bosons that on the heterotic side come from the Cartan subalgebra of the ten dimensional gauge group. At the particular point in moduli space where the $K 3$ is a $T^{4} / \mathbb{Z}_{2}$ orbifold, these sixteen gauge bosons arise from the twisted sectors of the orbifold, corresponding to states localized at the fixed points, one gauge field per fixed point.

Taking into account the normalization of the $R R$ fields in the type IIA action, and (3.1), the $t_{8} F^{4}$ term (2.7), which was a one loop effect on the heterotic side, must appear at tree level on the type IIA side. We can present some arguments for the non-renormalization of this term directly on the type IIA side: the identifiable BPS instantons would be Euclidean D0 and D2 branes, but there are no one-cycles or three-cycles for these branes to wrap. At the $T^{4} / \mathbb{Z}_{2}$ orbifold point of the moduli space, the tree level $t_{8} F^{4}$ terms in the type IIA string theory were successfully compared to the one-loop heterotic results in [46].

By tuning the moduli of the $K 3$, one can reach singular surfaces, which contain some two-cycles shrunk to zero size. These singularities of $K 3$ follow an $A D E$ classification. They provide the type IIA description of the enhanced ADE gauge symmetry that is visible perturbatively on the heterotic side. The W-bosons correspond in this description to $D 2$-branes wrapping the vanishing cycles. 
The expression (2.10) for the $t_{8} F^{4}$ term near a point of enhanced gauge symmetry was derived for the heterotic string under the condition $M_{W}^{2} \ll M_{h}^{2}$, but without any restriction on the value of $g_{h}$. For $g_{h} \gg 1$, the same expression must have a weakly coupled type IIA interpretation. To see what it is, recall that since the fundamental string on the heterotic side is given by the type IIA $N S 5$-brane wrapping the $K 3$, their tensions are related by $T_{h}=T_{N S 5} \operatorname{vol}(K 3) l_{I I}^{4}$, where $\operatorname{vol}(K 3)$ is the dimensionless volume of the $K 3$ in type II string units. Thus, $M_{h}^{2} \sim \operatorname{vol}(K 3) /\left(l_{I I}^{2} g_{s}^{2}\right)$, where $g_{s}$ is the ten dimensional IIA coupling, related to the IIA six dimensional coupling $g_{I I}$ by $g_{s}^{2}=g_{I I}^{2} \operatorname{vol}(K 3)$. Furthermore, in the type IIA theory the $\mathrm{W}$-bosons are wrapped $D 2$-branes, so $M_{W} \sim \operatorname{vol}\left(\right.$ two-cycle) $/\left(l_{I I} g_{s}\right)$, where vol(two-cycle) is the volume of the two-cycle the $D 2$-brane wraps, in string units. It follows that the condition for the validity of (2.10) on the type II side is

$$
\operatorname{vol}(\text { two-cycles }) \ll \sqrt{\operatorname{vol}(\mathrm{K} 3)} \text {. }
$$

This condition is purely geometric; in particular, it is independent of the type IIA string and Planck scales and of the type IIA string coupling.

We see that in the limit (3.2), the structure of the $t_{8} F^{4}$ terms is only sensitive to physics near the ADE singularity on the $K 3$ (through the volumes of the shrinking twocycles) and not to the detailed properties of the full theory. One can isolate that physics by studying the "near-horizon" region of the singularity; we will discuss this in more detail below.

It is interesting to note that unlike the heterotic case, in the weakly coupled type II limit $g_{s} \rightarrow 0$, there are actually two regimes that need to be analyzed separately. Denoting the string scale of the type II theory by $M_{s}\left(M_{s}=1 / l_{I I}\right)$, the physics is qualitatively different when $M_{W} \gg M_{s}$ and when $M_{W} \ll M_{s}$. The difference has to do with the fact that the W-bosons correspond to wrapped $D$-branes in the type II description. When $M_{W} \gg M_{s}$, they are very heavy, and a perturbative string description of the physics associated with the small cycles (3.2) is possible. This regime will be discussed in detail in $\S 4, \S 5$. It is there that the $t_{8} F^{4}$ interaction (2.10) must arise at tree level as discussed above, and we will show that it indeed does.

On the other hand, when $M_{W} \ll M_{s}$, the type II description is strongly coupled, and string perturbation theory breaks down, as is clear from the fact that in this regime the non-perturbative wrapped $D$-branes of mass $\simeq M_{W}$ are much lighter than perturbative string states. For the purposes of studying the $t_{8} F^{4}$ term (2.10) this region is actually 
simple, since the lightest massive states are the W-bosons, and their dynamics is given at low energies by $\mathcal{N}=(1,1)$ six dimensional SYM. Thus, the $t_{8} F^{4}$ term arises in this regime at one-loop, from the Feynman graphs discussed in $\S 2$, with the wrapped $D$-branes running in the loop.

So far, we have reviewed the duality between the heterotic string on $T^{4}$ and the type IIA string on $K 3$. There is a further duality relating these compactifications to a configuration of NS5-branes in type IIB string theory 47.48. To discuss this duality, consider a particular point in moduli space, type IIA on $T^{4} / \mathbb{Z}_{2}$. At this point in the moduli space the eighty moduli of the $K 3$ CFT split naturally into sixteen controlling the size and shape of the $T^{4} / \mathbb{Z}_{2}$, and the remaining sixty four, which correspond to blow up modes at the sixteen orbifold singularities.

Suppose, for simplicity, that the four-torus is a product of four circles, $T^{4}=\left(S^{1}\right)^{4}$. T-duality on one of the circles relates [48] this background to type IIB on $T^{4} / \mathbb{Z}_{2}$, where the $\mathbb{Z}_{2}$ acts in a non-standard way. A simple way of thinking about the resulting IIB background is as an S-dual of type IIB with sixteen orientifold five-planes and a D5-brane sitting at each of the 05 -planes (a T-dual description of type I string theory on $T^{4}$ ). Thus, each of the $\mathbb{Z}_{2}$ fixed points carries $(-1)$ units of $N S 5$-brane charge, which is canceled by an NS5-brane sitting at the fixed point. This type IIB vacuum does not have moduli that blow up the $\mathbb{Z}_{2}$ singularities. The original sixty four blow-up modes of the type IIA description map to moduli describing the positions of the sixteen NS5-branes on the $T^{4} / \mathbb{Z}_{2}$ (four real moduli per brane). At the orbifold point the sixteen $N S 5$-branes coincide with the fixed planes, but the duality holds also at generic points in the moduli space. In this realization, the $\mathrm{W}$-bosons are $D$-strings suspended between different NS5-branes; the enhanced gauge symmetry occurs when two or more NS5-branes are brought together.

\subsection{Duality for non-compact spaces}

In the previous subsection we described three dual realizations of the same physics. By varying the moduli, we can reach points with an enhanced gauge group. These gauge groups follow an $A D E$ classification, but their rank can be at most twenty four. However, if we consider local singularities embedded in a non-compact $K 3$ surface, we can realize configurations with arbitrary $A D E$ gauge group. Since eventually we will be interested in all possible LSTs with sixteen supercharges (which arise from decoupling limits of such singularities), we discuss this non-compact duality in some detail. 
For concreteness consider type IIA on a $\mathbb{C}^{2} / \mathbb{Z}_{k}$ singularity. The twisted sector includes $(k-1)$ six dimensional $\mathcal{N}=(1,1)$ vector multiplets, including NS-NS and RR fields, associated to the different two-cycles which vanish at the orbifold point. The moduli come from the NS-NS sector: the Kaluza-Klein reduction of the $B$ field on the two-cycles gives $(k-1)$ moduli $B_{i}$, which are singlets under the $S U(2)$ that rotates the complex structures; $(k-1)$ triplets of scalars $\vec{\zeta}_{i}$ account for metric deformations. Altogether, we have $4(k-1)$ real moduli.

This is related 14 by $T$-duality to $k N S 5$-branes of type IIB string theory arranged on a circle of radius $R$, in the limit

$$
g_{s}^{B} \rightarrow 0, \quad R / l_{I I}^{B} \rightarrow 0, \quad g_{s}^{A}=\frac{g_{s}^{B} l_{I I}^{B}}{R} \text { fixed } .
$$

The $(k-1) B_{i}$ are mapped to the relative distances between the $N S 5$-branes on the circle, while the $(k-1)$ triplets correspond to their transverse positions in the remaining $\mathbb{R}^{3}$. The limit of enhanced $S U(k)$ gauge symmetry in which the $B_{i} \rightarrow 0$ corresponds to bringing the $k N S 5$-branes together in the transverse $\mathbb{R}^{4}$ (the circle can be ignored in this limit).

We can regard this non-compact case as a limit of the compact case described in the previous subsection. Obviously, in this limit equation (3.2) is satisfied, so the heterotic dual satisfies $M_{W}^{2} \ll M_{h}^{2}$ and equation (2.10) should be valid.

For future reference, we recall that the type II solution for a collection of $k$ parallel $N S 5$-branes, at different points $\vec{x}_{n}$ in the transverse $\mathbb{R}^{4}$, is given by 16

$$
\begin{aligned}
& d s^{2}=-d t^{2}+d y_{1}^{2}+\ldots+d y_{5}^{2}+e^{2\left(D-D_{0}\right)}\left(d x_{6}^{2}+d x_{7}^{2}+d x_{8}^{2}+d x_{9}^{2}\right) ; \\
& e^{2\left(D-D_{0}\right)}=1+\sum_{n=1}^{k} \frac{\alpha^{\prime}}{\left(\vec{x}-\vec{x}_{n}\right)^{2}} ; \\
& H_{\mu \nu \lambda}=-\epsilon_{\mu \nu \lambda}^{\sigma} \partial_{\sigma} D .
\end{aligned}
$$

Here, $D$ is the dilaton, and $D_{0}$ is related to the asymptotic string coupling far from the $N S 5$-branes, $g_{s}=\exp \left(D_{0}\right) . H_{\mu \nu \lambda}$ is the field strength of the NS-NS $B$ field $B_{\mu \nu}$. When all the NS5-branes coincide, say at the origin, the transverse metric and dilaton near the branes are [16]

$$
\begin{aligned}
d s^{2} & =\frac{k \alpha^{\prime}}{r^{2}}\left(d r^{2}+r^{2} d \Omega_{3}^{2}\right)=d \phi^{2}+k \alpha^{\prime} d \Omega_{3}^{2}, \\
D & =-\frac{\phi}{\sqrt{k \alpha^{\prime}}}
\end{aligned}
$$

4 See 49] for a more detailed discussion. 
where $d \Omega_{3}^{2}$ is the metric on a unit three-sphere, and the new radial coordinate $\phi$ is defined by $e^{\phi / \sqrt{k \alpha^{\prime}}}=r e^{-D_{0}} / \sqrt{k \alpha^{\prime}}$. Note that in the limit 3.3 we take $g_{s}^{B} \rightarrow 0$. One might have naively thought that in this limit, the dynamics on the worldvolume of the NS5-branes becomes trivial, but as we review in the next section, that is not the case. The near-horizon limit (3.5) of the $k$ coincident $N S 5$-branes defines a non-trivial $5+1$ dimensional theory, a Little String Theory. Since the $t_{8} F^{4}$ terms are expected to be independent of the string coupling, one should be able to compute them in LST. We will devote the bulk of this paper to performing this computation and comparing it with the heterotic result (2.10).

\section{Type IIA string theory on a near-singular $K 3$}

As discussed in the previous section, when the $K 3$ surface develops two-cycles whose size is much smaller than that of the whole $K 3$, or in the T-dual picture, when the NS5branes approach each other to within a short distance, it is expected that the leading contribution to the $t_{8} F^{4}$ terms comes from the vicinity of the singularity. This is certainly true when the mass of the W-bosons, $M_{W}$, is much smaller than the string scale of the type II theory, $M_{s}$, since then the low-energy field theory approximation is valid. We will see below that it is also true for $M_{W} \gg M_{s}$, the region of interest here 5 . Thus, we are led to study the type II theory in a limit where we decouple the bulk of the $K 3$, and focus on the "near-horizon" geometry of the singularity. In this section we describe some features of this limit. We will often suppress constants and factors of $M_{s}$ in the discussion below, exhibiting them only when it seems necessary to do so.

\subsection{The decoupling limit and holography}

Near an $A_{k-1}$ ALE singularity 6 , the $K 3$ can be described as the surface

$$
z_{1}^{k}+z_{2}^{2}+z_{3}^{2}=0
$$

in $\mathbb{C}^{3}$. The manifold (4.1) contains a conical singularity at $z_{1}=z_{2}=z_{3}=0$. One can think of the overall scale of $\left(z_{1}, z_{2}, z_{3}\right)$ as the radial distance away from the singularity (we will make this more precise below).

5 This is natural, since the bulk of the $K 3$ cannot contribute terms that go like negative powers of $M_{W}$, such as those in equation (2.10).

6 For simplicity, we restrict in the present discussion to A-series singularities. It should be possible to generalize all of our results to the D- and E-series. 
Of course, (4.1) provides an accurate description of the geometry only very close to the singularity. In the full geometry, it has to be attached to the rest of the $K 3$. Since the bulk of the $K 3$ will not contribute to our calculations, we will neglect it and take the target space to be the non-compact $K 3$ surface (4.1) all the way to $z_{i}=\infty$.

Moreover, we would like to decouple any gravitational physics in the bulk of the ALE space. This can be achieved by sending $g_{s} \rightarrow 0$; by the duality described in $\S 3.2$, this limit in type IIA string theory is the same as the near-horizon limit of $k N S 5$-branes in type IIB string theory, and as described above, taking this limit does not affect the $t_{8} F^{4}$ terms we are interested in. Normally, string theory becomes free in the $g_{s} \rightarrow 0$ limit, but here this is not the case, since non-trivial dynamics remains in the vicinity of the singularity. This can be seen by embedding the ALE space (4.1) in a larger class of deformed spaces,

$$
z_{1}^{k}+z_{2}^{2}+z_{3}^{2}=\mu
$$

For $\mu \neq 0$ the conical singularity is smoothed out, and the formerly vanishing two-cycles get a finite volumel

$$
V_{S^{2}} \simeq \mu^{\frac{1}{k}}
$$

If one keeps the volume of the two-cycles fixed (and non-zero) as $g_{s} \rightarrow 0$, the theory becomes free, as is customary in string theory. To get a non-trivial theory, one can study the double-scaling limit [6]

$$
\begin{aligned}
& \mu \rightarrow 0, g_{s} \rightarrow 0, \\
& M_{W} \simeq \frac{\mu^{\frac{1}{k}}}{g_{s}}=\text { fixed },
\end{aligned}
$$

in which the Planck scale goes to infinity, but the mass of $D$-branes wrapped around the collapsing two-cycles remains finite. In effect, in this limit the scale $M_{W}$ replaces the Planck scale as the energy above which the theory becomes strongly coupled and non-perturbative effects become important. Since this scale is not associated with strong gravity effects, the resulting theory is non-gravitational - it is a Little String Theory. The above discussion also makes it clear that the non-trivial dynamics in the limit (4.4) is localized near the singularity, as explained in $\S 3$.

The double scaling limit (4.4) contains a tunable dimensionless parameter, $M_{W} / M_{s}$. If $M_{W} \ll M_{s}$ (an extreme case of which is the original ALE space (4.1)), string theory

7 This follows from the form of the holomorphic two-form on the deformed ALE space (4.2), $\Omega=d z_{1} \wedge d z_{2} / 2 z_{3}$. 
near the singularity becomes strongly coupled well below the string scale (at $E \simeq M_{W}$ ), and there are few useful tools for studying it except in the limit $E \rightarrow 0$, where it reduces to a (free) six dimensional $S U(k) \mathcal{N}=(1,1)$ supersymmetric Yang-Mills theory. This regime is of less interest to us, since the $t_{8} F^{4}$ terms are well understood in it (see $\S 2$ and $\S 3$ ). In the opposite limit, $M_{W} \gg M_{s}$, we expect a perturbative description to exist for energies $E \ll M_{W}$, and in particular for $E \sim M_{s}$. Thus, the situation here is similar to that in perturbative critical string theory (with the Planck scale replaced by $M_{W}$ ). This is the regime that we will study in the rest of this paper.

A very similar story can be told in the T-dual language of $N S 5$-branes in type IIB string theory on $T^{4} / \mathbb{Z}_{2}$. The T-dual of (4.1) involves $k$ coincident $N S 5$-branes at a regular point on $T^{4} / \mathbb{Z}_{2}$. To isolate the physics of the $N S 5$-branes, one proceeds as in the ALE case. First, focus on the geometry near the NS5-branes, taking the transverse space to be $\mathbb{R}^{4}$. This leads to the CHS metric (3.4). Then, send the (asymptotic) string coupling to zero. This gives rise to the background (see (3.5))

$$
\begin{aligned}
d s^{2} & =d x^{\mu} d x_{\mu}+k \alpha^{\prime}\left(d \sigma^{2}+d \Omega_{3}^{2}\right), \\
D & =-\sigma,
\end{aligned}
$$

where $\sigma$ is related to $r=|\vec{x}|$ of equation (3.4) by $r=\sqrt{k \alpha^{\prime}} g_{s} \exp \sigma$, and we have suppressed the $B$-field in (3.4).

The background (4.5) (with the appropriate $B$-field) can be described by an exact CFT,

$$
\mathbb{R}^{5,1} \times \mathbb{R}_{\phi} \times S U(2)_{k}
$$

where the real line $\mathbb{R}_{\phi}$ is labeled by $\phi \equiv \sqrt{k \alpha^{\prime}} \sigma$, the dilaton goes like

$$
D=-\frac{Q}{2} \phi ; \quad Q \equiv \frac{2}{\sqrt{k \alpha^{\prime}}}
$$

and the linear dilaton causes the central charge of the $\phi$ CFT to be

$$
c_{\phi}=1+\frac{3 \alpha^{\prime}}{2} Q^{2}=1+\frac{6}{k}
$$

The supersymmetric $S U(2)_{k}$ CFT consists of a level $(k-2)$ bosonic $S U(2)$ WZW model, as well as three free fermions $\psi_{a}, a=1,2,3$, which transform in the adjoint of an $S U(2)_{2}$ affine Lie algebra, completing the total level of $S U(2)$ to $k$. There are also free fermions $\psi_{\mu}$, $\mu=0,1, \cdots, 5$, and $\psi_{\phi}$, which are the worldsheet superpartners of $x_{\mu}$ and $\phi$, respectively. 
The ADE classification of singularities of $K 3$ surfaces is mapped in the description (4.6) to the ADE classification of $S U(2)$ modular invariants.

Geometrically, the different components of (4.6) can be thought of as follows. $\mathbb{R}^{5,1}$ is the worldvolume of the $N S 5$-branes, $\mathbb{R}_{\phi}$ parameterizes the radial direction away from the branes, while $S U(2)_{k}$ describes the angular three-spheres at constant distance from the branes. The $S U(2)_{L} \times S U(2)_{R}$ symmetry associated with the $S U(2)_{k} \mathrm{CFT}$ is identified with the $S O(4)$ rotation symmetry around the $N S 5$-branes.

The background (4.6) can be understood from the ALE point of view as well [47,26]. By writing

$$
\left(z_{1}, z_{2}, z_{3}\right)=\left(\lambda^{\frac{2}{k}} y_{1}, \lambda y_{2}, \lambda y_{3}\right)
$$

where $\lambda=r \exp (i \theta) \in \mathbb{C}$, and $\left(y_{1}, y_{2}, y_{3}\right)$ take values in a weighted complex projective space, one can argue that $r$ parameterizes the radial direction $\phi$ in (4.6), $\theta$ labels the Cartan subgroup of $S U(2)_{k}$, while $\left(y_{1}, y_{2}, y_{3}\right)$, which satisfy (4.1) as well, parameterize the coset $S U(2)_{k} / U(1)$. Thus, the ALE description corresponds to the parametrization

$$
\mathbb{R}_{\phi} \times S U(2)_{k} \simeq \mathbb{R}_{\phi} \times\left(S_{k}^{1} \times \frac{S U(2)_{k}}{U(1)}\right) / \mathbb{Z}_{k}
$$

The radius of $S_{k}^{1}$ is $\sqrt{\alpha^{\prime} k}$. An important fact is that (4.6), (4.10) are only valid for $k \geq 2$, i.e. for two or more coincident NS5-branes. For $k=1$, the ALE space (4.1) is smooth and, in the T-dual language, a single NS5-brane does not develop a throat.

The near-horizon description of the singularity (4.6) is strongly coupled; the string coupling $g_{s}=e^{D}$ diverges as $\phi \rightarrow-\infty$, which corresponds to the location of the NS5branes, or the tip of the cone in ALE. This behavior is in agreement with our general considerations above, since (4.6) corresponds to the case $\mu=0$ in (4.2), or $M_{W}=0$ in (4.4). Thus the theory is strongly coupled for any finite energy, and the weakly coupled description for low energy is not via string theory on (4.6), but rather via supersymmetric Yang-Mills theory.

In order to arrive at a weakly coupled string theory description, we would like to separate the $N S 5$-branes in the transverse space, or equivalently smooth out the tip of the cone, as in (4.2). This is expected to eliminate the strong coupling singularity 8 in (4.6), and introduce the tunable parameter, $M_{W} / M_{s}$, into the problem. To do that it is useful

8 In the $N S 5$-brane picture, this is because as mentioned after equation (4.10), a single $N S 5$ brane does not have a throat along which the string coupling can diverge. 
to have in mind the holographic interpretation of the background (4.6), to which we turn next.

In [3] it was proposed that string theory in asymptotically linear dilaton space-times, such as (4.6), is holographically related to a dual theory, a Little String Theory. This relation is analogous to that of the AdS/CFT correspondence [50]. The analogy is most straightforward in the $N S 5$-brane picture, where the duality relates a decoupled theory on the world-volume of a stack of $N S 5$-branes, with string theory in the near-horizon geometry of the branes. The $\phi$ direction in (4.6) is expected to play the role of the holographic direction, while the $S^{3}$ is associated with the $S O(4)$ global symmetry of the LST. Observables correspond to non-normalizable vertex operators 8 whose wavefunctions diverge at the weakly coupled "boundary" $\phi \rightarrow \infty$. Correlation functions of such operators correspond to off-shell Green's functions in the dual LST. We will be interested here in observables corresponding to short representations of space-time supersymmetry, which as discussed in [3], are in one to one correspondence with gauge-invariant operators in short representations of supersymmetry in the low-energy gauge theory.

The low energy limit of the LST contains $S U(k) \mathcal{N}=(1,1)$ SYM theory, and we can label the operators of the LST using their descriptions as operators in this gauge theory. An example of the correspondence between LST operators and non-normalizable vertex operators that will be useful below is:

$$
\widetilde{\operatorname{tr}}\left(\Phi^{i_{1}} \Phi^{i_{2}} \cdots \Phi^{i_{2 j+2}}\right) \leftrightarrow e^{-\varphi-\bar{\varphi}}\left(\psi \bar{\psi} \Phi_{j}^{(s u)}\right)_{j+1 ; m, \bar{m}} e^{Q \tilde{\jmath} \phi} e^{i p \cdot x}
$$

for $j=0,1 / 2,1, \cdots,(k-2) / 2$, where $\Phi^{i}, i=6,7,8,9$ are the four scalar fields in the adjoint of $S U(k)$, which parameterize the locations of the $N S 5$-branes in the transverse directions, and on the left-hand side of (4.11) one should consider only the symmetric, traceless components in $\left(i_{1}, i_{2}, \cdots, i_{2 j+2}\right)$ (this is required for the operator to be in a short representation). On the right-hand side of (4.11), $\varphi, \bar{\varphi}$ are the bosonized superconformal ghosts, $\Phi_{j ; m, \bar{m}}^{(s u)}$ is a primary of the bosonic $S U(2)_{k-2}$ WZW model10, and the notation $\left(\psi \bar{\psi} \Phi_{j}^{(s u)}\right)_{j+1 ; m, \bar{m}}$ means that we are coupling the fermions in the adjoint of $S U(2), \psi^{a}$, with the bosonic part into a primary of total spin $(j+1)$ and $\left(J_{3}^{\text {tot }}, \bar{J}_{3}^{\text {tot }}\right)=(m, \bar{m})$. The

9 A second class of observables corresponds to $\delta$-function normalizable vertex operators. We will not discuss those here.

10 We review some properties of this model in appendix B. 
values of $m$ and $\bar{m}$ depend on the precise indices appearing on the left-hand side. The mass shell condition provides a relation between $\tilde{\jmath}, j$ and $p_{\mu}$, as we review below.

The notation $\widetilde{\operatorname{tr}}$ refers to the fact that the operator in question has the same quantum numbers as the trace, but it is not precisely equal to the trace. Rather, it is a combination of the single-trace operator with multi-trace operators such as $\operatorname{tr}\left(\Phi^{i_{1}} \Phi^{i_{2}}\right) \operatorname{tr}\left(\Phi^{i_{3}} \cdots \Phi^{i_{2 j+2}}\right)$. Such a mixing occurs quite generally in holographic dualities such as the AdS/CFT correspondence, but usually one is only interested in the limit $j \ll k$ where the multi-trace contributions are negligible. In this paper we are not taking the large $k$ limit, so the distinction is in principle important. When $j \sim k$ it is known in various examples that specific combinations such as subdeterminants appear [51-53. The precise combinations of single and multi-trace operators that correspond to single string vertex operators in our case have been determined in [54 11. However, at the particular point in moduli space we will be working, the multi-trace components of (4.11) will not contribute to the specific computations we will do in $\S 5, \S 6$, and we can treat these operators as single-trace operators. We will normalize the expression $\widetilde{t r}$ such that it is equal to the single-trace operator with coefficient one, plus multi-trace operators. Thus, for the purposes of $\S 5, \S 6$ of this paper, one can replace $\widetilde{\operatorname{tr}} \rightarrow \operatorname{tr}$ in all expressions.

The mass-shell condition for the vertex operator (4.11) reads

$$
Q^{2}(\tilde{\jmath}-j)(\tilde{\jmath}+j+1)=p^{2}
$$

with the larger root $\tilde{\jmath}$ of this equation corresponding to the non-normalizable vertex operator. The statement of holography is that correlation functions of the vertex operators (4.11) in the bulk theory correspond to off-shell Green's functions of the operators $\widetilde{\operatorname{tr}}\left(\Phi^{i_{1}} \Phi^{i_{2}} \cdots \Phi^{i_{2 j+2}}\right)$ in the UV completion of six dimensional super Yang-Mills theory provided by LST.

11 In different contexts it may be more natural to choose the single string vertex operators to correspond precisely to single-trace operators. It is well-known that different choices of contact terms in a worldsheet CFT lead to different parametrizations of the space of couplings [55]. We believe that with different choices of such contact terms we can change the multi-trace content of the operators (4.11) (which are related to couplings as described below) and go between the theory we describe here and the theory in which they are single-traces. 
Another useful example of the correspondence is obtained by acting on (4.11) once with a chiral and once with an anti-chiral space-time supercharge in a way which creates a two-form operator (with polarization $\zeta^{\mu \nu}$ ),

$$
\begin{aligned}
& \zeta^{\mu \nu}\left[\tilde{\operatorname{tr}}\left(F_{\mu \nu} \Phi^{i_{1}} \Phi^{i_{2}} \cdots \Phi^{i_{2 j+1}}\right)\right.\left.+\widetilde{\operatorname{tr}}\left(\lambda \gamma_{\mu \nu} \bar{\lambda} \Phi^{i_{1}} \cdots \Phi^{i_{2 j}}\right)\right] \leftrightarrow \\
& \zeta^{\mu \nu} e^{-\frac{1}{2}(\varphi+\bar{\varphi})} S_{a} \gamma_{\mu \nu}^{a \dot{a}} \bar{S}_{\dot{a}}\left(S \bar{S} \Phi_{j}^{(s u)}\right)_{j+\frac{1}{2} ; m, \bar{m}} e^{Q j \phi}
\end{aligned}
$$

On the left-hand side, $\lambda$ is a gaugino which transforms in the $(\mathbf{4}, \mathbf{2})$ representation of $\operatorname{Spin}(5,1) \times \operatorname{Spin}(4) ; \bar{\lambda}$ transforms in the $(\overline{\mathbf{4}}, \overline{\mathbf{2}})$. The $\mathrm{RR}$ vertex operator on the right-hand side is written for $p_{\mu}=0$; the general form is more complicated (and will appear below for a special case). $\left(S \bar{S} \Phi_{j}^{(s u)}\right)_{j+\frac{1}{2} ; m, \bar{m}}$ corresponds to the coupling of the spin fields constructed out of the worldsheet fermions $\psi_{1}, \psi_{2}, \psi_{3}, \psi_{\phi}$ (and their antiholomorphic counterparts), which transform in the spin 1/2 representation of $S U(2)_{L}\left(S U(2)_{R}\right)$, with the spin $j$

operator $\Phi_{j}^{(s u)}$, into an operator transforming in the spin $(j+1 / 2)$ representation. The spin fields $S_{a}$ and $\bar{S}_{\dot{a}}$ transform as the $\mathbf{4}$ and $\overline{\mathbf{4}}$ of $\operatorname{Spin}(5,1)$, respectively.

To verify that the operators (4.11), (4.13) are BRST invariant, as well as for our subsequent analysis, one needs to use the detailed structure of the CFT corresponding to the CHS background (4.6). We summarize some of the relevant results in appendix A.

\subsection{The moduli space of deformed ALE spaces}

As discussed in the previous subsection, to get a weakly coupled worldsheet description of ALE spaces or NS5-branes, we must deform the singularity, or equivalently separate the NS5-branes. In this subsection, we discuss the relevant deformation from the point of view of string theory in the CHS background (4.6).

From the low-energy field theory point of view, we would like to give the scalars $\Phi^{i}$ (see (4.11)) VEVs of the form

$$
\left\langle\Phi^{i}\right\rangle=\operatorname{diag}\left(\phi_{1}^{i}, \phi_{2}^{i}, \cdots, \phi_{k}^{i}\right)
$$

where $\phi_{n}^{i}, i=6,7,8,9, n=1,2, \cdots, k$, is the location of the $n$ 'th $N S 5$-brane in the $i$ 'th direction. Since the SYM potential is proportional to $\operatorname{tr}\left[\Phi^{i}, \Phi^{j}\right]^{2}$, (4.14) are indeed flat directions of the potential. The NS5-brane picture makes it clear that (4.14) are flat directions even when the VEVs $\phi_{n}^{i}$ are large. Since the low-energy gauge group is $S U(k)$ (the "center of mass" $U(1)$ is not part of the decoupled interacting theory) we will set $\sum_{n} \phi_{n}^{i}=0$. 
How do we describe the VEVs (4.14) in the CHS background? The vertex operators corresponding to chiral gauge-invariant combinations of the $\Phi$ 's are given in (4.11) 12 . Adding the vertex operators on the right-hand side of (4.11) to the worldsheet Lagrangian corresponds, in the low-energy field theory, to adding the operators on the left-hand side of (4.11) to the space-time Lagrangian. As is well-known in the context of the AdS/CFT correspondence [56], if instead we want to give expectation values to the operators (4.11), we have to add to the worldsheet Lagrangian the normalizable versions of the vertex operators (4.11), which are obtained by sending $\tilde{\jmath} \rightarrow-\tilde{\jmath}-1$ in $(4.11) 13$. Thus, we are led to consider worldsheet Lagrangians of the form

$$
\mathcal{L}=\mathcal{L}_{0}+\lambda_{j ; m, \bar{m}} G_{-\frac{1}{2}} \bar{G}_{-\frac{1}{2}}\left(\psi \bar{\psi} \Phi_{j}^{(s u)}\right)_{j+1 ; m, \bar{m}} e^{-Q(j+1) \phi}
$$

where $\mathcal{L}_{0}$ is the Lagrangian describing the CHS background, $G$ is the supercurrent defined in appendix A, and the couplings $\lambda_{j ; m, \bar{m}}$ are determined by the values of the moduli (4.14). Note that we have shifted the operator to the $(0,0)$ picture and that we have set the spacetime momentum $p_{\mu}=0$, since we are interested in describing a condensate that is constant in space-time.

The number of couplings $\lambda_{j ; m, \bar{m}}$ in (4.15) is in general larger than the number of parameters determining the point in moduli space. Indeed, the former goes like $k^{3}$ for large $k$, while the latter is equal to $4(k-1)$. Thus, it must be that in order to obtain a sensible worldsheet theory from (4.15), one has to impose relations on the $\lambda$ 's, which follow from the fact that they are functions of the $\phi_{n}^{i}$ (4.14). We will see later an example of such a relation which is understood in the worldsheet theory. In general, the origin of such relations is not fully understood from the worldsheet point of view.

Note also that adding the terms in (4.15) to the worldsheet Lagrangian does not modify the background near the boundary at $\phi \rightarrow \infty$, but as $\phi \rightarrow-\infty$, the new terms grow and regularize the divergence coming from the strong coupling region in the CHS solution. This is in agreement with the target space picture. Far away from the tip of the ALE cone, or from the locations of the NS5-branes (but still in the near-horizon geometry), one cannot tell whether the singularity has been smoothed out or not. Upon approaching

12 In the low-energy field theory non-chiral operators also obtain vacuum expectation values in the configuration (4.14). The corresponding vertex operators in string theory are not known, but they do not seem to play an important role for the purposes of this paper.

13 The precise definition and meaning of these operators will be described in $§ 5.2$. 
the singularity, one notices that the $\left\{\phi_{n}^{i}\right\}$ have been turned on, and the singularity has been smoothed out.

Since the general case is complicated, we next (following [7]) restrict the discussion to a subspace of the moduli space (4.14), by restricting the $N S 5$-branes to move in a plane. Thus, we denote

$$
\begin{aligned}
& A \equiv \Phi^{6}+i \Phi^{7}, \\
& B \equiv \Phi^{8}+i \Phi^{9}
\end{aligned}
$$

and keep $\langle A\rangle=0$ while varying

$$
\langle B\rangle=\operatorname{diag}\left(b_{1}, b_{2}, \cdots, b_{k}\right) ; \sum_{n=1}^{k} b_{n}=0 .
$$

It is convenient to embed the $S O(2)_{A} \times S O(2)_{B}$ symmetries of rotations of the $A, B$ planes as follows in the $S U(2)_{L} \times S U(2)_{R}$ symmetry of the CHS background. The generator of $S O(2)_{A}$ will be taken to be $J_{3}^{\text {tot }}-\bar{J}_{3}^{\text {tot }}$, while that of $S O(2)_{B}$ is $J_{3}^{\text {tot }}+\bar{J}_{3}^{\text {tot }}$. The charges are normalized such that

$$
\begin{aligned}
& \left(J_{3}^{\text {tot }}+\bar{J}_{3}^{\text {tot }}\right)(A)=0 ; \quad\left(J_{3}^{\text {tot }}-\bar{J}_{3}^{\text {tot }}\right)(A)=1 ; \\
& \left(J_{3}^{\text {tot }}+\bar{J}_{3}^{\text {tot }}\right)(B)=1 ; \quad\left(J_{3}^{\text {tot }}-\bar{J}_{3}^{\text {tot }}\right)(B)=0
\end{aligned}
$$

Then, one finds that (at zero space-time momentum),

$$
\begin{aligned}
\operatorname{tr}\left(A^{l} B^{2 j+2-l}\right) & \leftrightarrow e^{-\varphi-\bar{\varphi}}\left(\psi \bar{\psi} \Phi_{j}^{(s u)}\right)_{j+1 ; j+1, j+1-l} e^{Q j \phi}, \\
\tilde{\operatorname{tr}}\left(A^{l}\left(B^{*}\right)^{2 j+2-l}\right) & \leftrightarrow e^{-\varphi-\bar{\varphi}}\left(\psi \bar{\psi} \Phi_{j}^{(s u)}\right)_{j+1 ;-j-1+l,-j-1} e^{Q j \phi},
\end{aligned}
$$

and in particular

$$
\widetilde{\operatorname{tr}}\left(B^{2 j+2}\right) \leftrightarrow e^{-\varphi-\bar{\varphi}} \psi^{+} \bar{\psi}^{+} \Phi_{j ; j, j}^{(s u)} e^{Q j \phi}=e^{-\varphi-\bar{\varphi}} e^{i(H+\bar{H})} \Phi_{j ; j, j}^{(s u)} e^{Q j \phi} .
$$

We will also be interested later in the corresponding operators for (4.13). One has

$$
\mathcal{O}_{2 j+1}^{+} \equiv \zeta^{\mu \nu} \widetilde{\operatorname{tr}}\left(F_{\mu \nu} B^{2 j+1}+\text { fermions }\right) \leftrightarrow \zeta^{\mu \nu} e^{-\frac{1}{2}(\varphi+\bar{\varphi})} S_{a} \gamma_{\mu \nu}^{a \dot{a}} \bar{S}_{\dot{a}} e^{\frac{i}{2}\left(H+H^{\prime}+\bar{H}+\bar{H}^{\prime}\right)} \Phi_{j ; j, j}^{(s u)} e^{Q j \phi}
$$

The field $\psi^{+}$and the bosonized fermions $H, H^{\prime}, \bar{H}$ and $\bar{H}^{\prime}$, appearing in (4.20) and (4.21), are defined in appendix $\mathrm{A}$.

In order to describe condensation of $B$, as in (4.17), we are led to study the perturbed worldsheet Lagrangian

$$
\mathcal{L}=\mathcal{L}_{0}+\sum_{j}\left(\lambda_{j} G_{-\frac{1}{2}} \bar{G}_{-\frac{1}{2}} \psi^{+} \bar{\psi}^{+} \Phi_{j ; j, j}^{(s u)} e^{-Q(j+1) \phi}+\text { c.c. }\right)
$$


These perturbations are particularly nice, since the operators $\psi^{+} \bar{\psi}^{+} \Phi_{j ; j, j}^{(s u)} e^{-Q(j+1) \phi}$ are chiral. Indeed, by using the form of the $\mathcal{N}=2$ superconformal generators $G^{ \pm}$given in appendix $\mathrm{A}$, one can show that

$$
G^{+}(z) \psi^{+} \bar{\psi}^{+} \Phi_{j ; j, j}^{(s u)} e^{-Q(j+1) \phi}(w)=\text { regular as } z \rightarrow w
$$

and similarly for $\bar{G}^{+}$. Thus, one can think of the perturbations in (4.22) as turning on a worldsheet superpotential. A useful way of thinking about this is in terms of the decomposition (4.10). We have an infinite cylinder $\mathbb{R}_{\phi} \times S_{k}^{1}$, labeled by $\phi$ and $Y$, where $Y$ is defined by

$$
J_{3}^{\text {tot }}=\frac{i}{Q} \partial Y
$$

and $Y$ is canonically normalized. Here, and in the rest of the section, we set $\alpha^{\prime}=2$. $S U(2)_{k} / U(1)$ is an $\mathcal{N}=2$ minimal model, which can be described in terms of a LandauGinzburg superfield $\chi$, with superpotential

$$
W=\chi^{k}
$$

In these variables, one can write

$$
\psi^{+} \bar{\psi}^{+} \Phi_{j ; j, j}^{(s u)} e^{-Q(j+1) \phi}=\chi^{k-2(j+1)} e^{-Q(j+1)(\phi-i Y)}=\chi^{k-2(j+1)} e^{-Q(j+1) \Phi}
$$

where in the last step we have defined a chiral superfield whose bottom component is $\Phi=\phi-i Y$ (following standard practice, we will denote both the superfield and its bottom component by $\Phi$, and similarly for $\chi)$. The Lagrangian (4.22) can be written as

$$
\mathcal{L}=\mathcal{L}_{0}+\sum_{j}\left(\lambda_{j} \int d^{2} \theta \chi^{k-2(j+1)} e^{-Q(j+1) \Phi}+\text { c.c. }\right)
$$

One can use the correspondence (4.20) to relate the $\left\{\lambda_{j}\right\}$ to the locations of the $k N S 5$ branes in the $B$-plane:

$$
\lambda_{j} \sim\left\langle\tilde{\operatorname{tr}}\left(B^{2 j+2}\right)\right\rangle
$$

While the structure of the theory on the full moduli space labeled by $\left\{\lambda_{j}\right\}$ (4.27) is of interest, and can probably be analyzed using our techniques, we will further specialize to the subspace of moduli space corresponding to $N S 5$-branes which are equally spaced on a circle in the $B$-plane of radius $r_{0}$,

$$
b_{n}=r_{0} e^{2 \pi i n / k}
$$


At this particular point in moduli space, $\operatorname{tr}\left\langle B^{l}\right\rangle=0$ for all $l<k$, so all the possible multitrace terms, such as $\operatorname{tr}\left\langle B^{l}\right\rangle \operatorname{tr}\left\langle B^{k-l}\right\rangle$ vanish, and there is no difference between evaluating the VEVs of the operators $\tilde{\operatorname{tr}}\left(B^{l}\right)$ defined in (4.11) and the ordinary single-trace operators $\operatorname{tr}\left(B^{l}\right)$. In this case we have, using (4.28),

$$
\lambda_{j}=\mu \delta_{j, \frac{k-2}{2}}
$$

with

$$
\mu \sim r_{0}^{k}
$$

and the deformed ALE space is described by (4.2). The worldsheet theory (4.27) simplifies in this case, since the perturbation decouples from the $\mathcal{N}=2$ minimal model:

$$
\mathcal{L}=\mathcal{L}_{0}+\left(\mu \int d^{2} \theta e^{-\frac{1}{Q} \Phi}+\text { c.c. }\right)
$$

The resulting worldsheet theory is $\mathcal{N}=2$ Liouville times an $\mathcal{N}=2$ minimal model (with a $\mathbb{Z}_{k}$ identification (4.10)). The exponential superpotential cuts off the strong coupling divergence of the string coupling $g_{s}$ as $\phi \rightarrow-\infty$.

It is known that $\mathcal{N}=2$ Liouville is equivalent as a CFT to the coset $S L(2)_{k} / U(1)$, which describes string propagation on a cigar of asymptotic radius $\sqrt{2 k}[6,57,58,59]$. In the cigar description of this theory, the strong coupling region $\phi \rightarrow-\infty$ is excised altogether, and as long as $g_{s}$ at the tip of the cigar $g_{s}^{(\text {tip) }}$ (which scales as $\mu^{-1 / k}$ ) remains small, the theory is weakly coupled 14 .

In fact, the space-time point of view on the deformations (4.15) sheds interesting light on the duality between the $\mathcal{N}=2$ Liouville and cigar CFTs. In our discussion of the condensate (4.29) we focused on the expectation values of the chiral operators $\widetilde{\operatorname{tr}}\left(B^{2 j+2}\right)$, but the VEV (4.29) leads to non-zero expectation values of other chiral operators as well. For example, the chiral operator $\operatorname{tr}\left(B B^{*}-A A^{*}\right)$, which is a special case of the right-hand side of (4.11), has a VEV:

$$
\left\langle\widetilde{\operatorname{tr}}\left(B B^{*}-A A^{*}\right)\right\rangle=k r_{0}^{2}
$$

According to the dictionary (4.11), (4.15), this corresponds to turning on the perturbation

$$
\delta \mathcal{L} \sim k r_{0}^{2} G_{-\frac{1}{2}} \bar{G}_{-\frac{1}{2}} \psi_{3} \bar{\psi}_{3} e^{-Q \phi}+\text { c.c. }
$$

14 Recall that the string coupling is largest at the tip of the cigar. 
in the worldsheet sigma model. This perturbation is well-known to be the leading term in the expansion of the metric of the cigar around $\phi=\infty$, the region far from the tip. The higher order terms in that expansion correspond to VEVs of higher order chiral operators, involving higher powers of $\left(B B^{*}\right)$ and $\left(A A^{*}\right)$.

Thus, we see that from the space-time point of view, both the cigar and $\mathcal{N}=2$ Liouville perturbations are present in the worldsheet Lagrangian corresponding to (4.29), with related coefficients. For some purposes, one can focus on the superpotential terms (4.27); for others, the cigar picture (including (4.34)) is more useful. In general, both have to be taken into account. The relation between the two deformations will become clearer when we discuss relations between normalizable operators in the next section. All this ties in nicely to the worldsheet analysis of these theories. For example, the relation between the Liouville and cigar perturbations was determined in [58].

At the special point in moduli space that we are now discussing (4.29), the throat CFT becomes 47

$$
\left(S L(2)_{k} / U(1) \times S U(2)_{k} / U(1)\right) / \mathbb{Z}_{k}
$$

The wrapped $D 2$-branes discussed earlier in this section correspond to $D$-branes localized near the tip of the cigar, times various $D$-branes in the $\mathcal{N}=2$ minimal models [60]. Their mass is proportional to $r_{0}$ and satisfies

$$
M_{W} \simeq \frac{M_{s}}{g_{s}^{(\mathrm{tip})}} \gg M_{s}
$$

They are heavy non-perturbative objects in the limit we are studying, as indicated in equation (4.36).

\subsection{Vertex operators in the $\left(S L(2)_{k} / U(1) \times S U(2)_{k} / U(1)\right) / \mathbb{Z}_{k}$ background}

In order to calculate correlation functions in the deformed background (4.35), one has to know what the different observables of string theory on the CHS background, discussed above, correspond to upon the resolution of the singularity. This can be understood using standard CFT techniques. For example, the vertex operator of $\widetilde{\operatorname{tr}}\left(B^{2 j+2}\right)$, (4.20), corresponds in the deformed background (4.35) to

$$
e^{i(H+\bar{H})} \Phi_{j ; j, j}^{(s u)} e^{Q j \phi} \leftrightarrow V_{\frac{k}{2}-j-1 ;-\frac{k}{2}+j+1,-\frac{k}{2}+j+1}^{(s u, s u s y)} V_{j ; j+1, j+1}^{(s l, s u s y)}
$$


where $V_{j ; m, m}^{(\text {su,susy })}$ is a primary in the $\mathcal{N}=2$ minimal model with dimension and R-charge

$$
\Delta=\bar{\Delta}=\frac{j(j+1)-m^{2}}{k} ; \quad R=\bar{R}=-\frac{2 m}{k}
$$

while $V_{j ; m, m}^{(s l, s u s y)}$ is an $\mathcal{N}=2$ primary in $S L(2)_{k} / U(1)$; it has

$$
\Delta=\bar{\Delta}=\frac{m^{2}-j(j+1)}{k} ; \quad R=\bar{R}=\frac{2 m}{k}
$$

Similarly, for the RR operators 4.21 one has

$$
e^{\frac{i}{2}\left(H+H^{\prime}+\bar{H}+\bar{H}^{\prime}\right)} \Phi_{j ; j, j}^{(s u)} e^{Q j \phi} \leftrightarrow V_{j ; j, j}^{(s u, s u s y)}(R R,+) V_{j ; j, j}^{(s l, s u s y)}(R R,+)
$$

where $V_{j ; m, m}^{(\text {su,susy })}(R R, \pm)$ is a RR sector operator in the $\mathcal{N}=2$ minimal model; it has

$$
\Delta=\bar{\Delta}=\frac{1}{8}+\frac{j(j+1)-\left(m \pm \frac{1}{2}\right)^{2}}{k} ; \quad R=\bar{R}= \pm \frac{1}{2}-\frac{2 m \pm 1}{k}
$$

while $V_{j ; m}^{(s l, s u s y)}(R R, \pm)$ is a similar object in the cigar CFT, which has

$$
\Delta=\bar{\Delta}=\frac{1}{8}-\frac{j(j+1)-\left(m \pm \frac{1}{2}\right)^{2}}{k} ; \quad R=\bar{R}= \pm \frac{1}{2}+\frac{2 m \pm 1}{k}
$$

Thus, $V_{j ; j, j}^{(\text {su,susy })}(R R,+)$ has dimension $\Delta=\frac{1}{8}-\frac{1}{4 k}$ and corresponds to a RR ground state in the $\mathcal{N}=2$ minimal model (which has $\left.c=3-\frac{6}{k}\right)$. Similarly, $V_{j ; j, j}^{(s l, s u s y)}(R R,+$ ) has dimension $\Delta=\frac{1}{8}+\frac{1}{4 k}$ and corresponds to a RR ground state of the cigar CFT.

As we will see in the next section, in our analysis we will also need to use operators whose asymptotic form for large $\phi$ is

$$
e^{\frac{i}{2}\left(H-H^{\prime}+\bar{H}-\bar{H}^{\prime}\right)} \Phi_{j ; j, j}^{(s u)} e^{Q j \phi} \text { or } e^{\frac{i}{2}\left(H-H^{\prime}+\bar{H}-\bar{H}^{\prime}\right)} \Phi_{j ; j, j}^{(s u)} e^{Q(-j-1) \phi}
$$

In the deformed background (4.35), they correspond to

$$
\begin{aligned}
e^{\frac{i}{2}\left(H-H^{\prime}+\bar{H}-\bar{H}^{\prime}\right)} \Phi_{j ; j, j}^{(s u)} e^{Q j \phi} & \leftrightarrow V_{j ; j, j}^{(\text {su,susy })}(R R,+) V_{j ; j+1, j+1}^{(s l, s u s y)}(R R,-), \\
e^{\frac{i}{2}\left(H-H^{\prime}+\bar{H}-\bar{H}^{\prime}\right)} \Phi_{j ; j, j}^{(s u)} e^{Q(-j-1) \phi} & \leftrightarrow V_{j ; j, j}^{(s u, \text { susy })}(R R,+) V_{-j-1 ; j+1, j+1}^{(s l, s u s y)}(R R,-) .
\end{aligned}
$$

The operator $V_{-j-1 ; j+1, j+1}^{(\text {sl,susy }}(R R,-)$, whose precise definition will be discussed in $\S 5.2$ below, has the same dimension as the operator $V_{j ; j, j}^{(s l, s u s y)}(R R,+)$, and also corresponds to a ground state of the cigar CFT. 
It is sometimes useful to bosonize the $\mathcal{N}=2$ minimal model, by using its description as a product of a bosonic $S U(2) / U(1)$ (parafermion) theory and a compact canonically normalized scalar field (which we will label by $P$ ) [61]. The NS-NS sector $\mathcal{N}=2$ primaries can be written as

$$
V_{j ; m, m}^{(s u, s u s y)}=V_{j ; m, m}^{(s u)} e^{i \alpha_{m} P}
$$

where $V_{j ; m, m}^{(s u)}$ is a primary in the parafermion theory whose dimension is given by

$$
\Delta\left(V_{j ; m, m}^{(s u)}\right)=\bar{\Delta}\left(V_{j ; m, m}^{(s u)}\right)=\frac{j(j+1)}{k}-\frac{m^{2}}{k-2}
$$

and

$$
\alpha_{m}=\frac{2 m}{\sqrt{k(k-2)}} .
$$

Indeed one can check using (4.38), (4.45) and (4.46) that

$$
\Delta\left(V_{j ; m, m}^{(s u, s u s y)}\right)=\Delta\left(V_{j ; m, m}^{(s u)}\right)+\frac{1}{2} \alpha_{m}^{2} .
$$

Note that $\alpha_{m}$ is related to the R-charge of the $V_{j ; m, m}^{(s u)}$ as follows:

$$
R^{(s u)}=-\alpha_{m} \sqrt{1-\frac{2}{k}}=-\frac{2 m}{k} .
$$

In fact, the scalar $P$ can be thought of as a bosonized version of the $U(1)_{R}$ current of the $S U(2) / U(1) \mathrm{SCFT}$. In the RR sector one has

$$
V_{j ; m, m}^{(s u, s u s y)}(R R, \pm)=V_{j ; m, m}^{(s u)} e^{i \alpha_{m}^{ \pm} P}
$$

where

$$
\alpha_{m}^{ \pm}=\frac{2 m \mp \frac{1}{2}(k-2)}{\sqrt{k(k-2)}} .
$$

One can check using (4.41), (4.46), (4.51), that

$$
\Delta\left(V_{j ; m, m}^{(s u, s u s y)}(R R, \pm)\right)=\Delta\left(V_{j ; m, m}^{(s u)}\right)+\frac{1}{2}\left(\alpha_{m}^{ \pm}\right)^{2}
$$

The relation between the R-charge of the operator $V_{j ; m}^{(s u, s u s y)}(R R, \pm)$ and the $P$ charge $\alpha_{m}^{ \pm}$ is again (as in (4.49))

$$
R^{ \pm(s u)}=-\alpha_{m}^{ \pm} \sqrt{1-\frac{2}{k}}= \pm \frac{1}{2}-\frac{2 m \pm 1}{k} .
$$


A useful relation between the $(R R,+)$ and $(R R,-)$ operators is

$$
V_{j ; m, m}^{(\text {su,susy })}(R R,+)=V_{\frac{k-2}{2}-j ;-\frac{k-2}{2}+m,-\frac{k-2}{2}+m}^{(\text {su,susy })}(R R,-) .
$$

This reflection property follows from (4.51) and from the well-known 62 property of the parafermion theory

$$
V_{j ; m, m}^{(s u)}=V_{\frac{k-2}{2}-j ;-\frac{k-2}{2}+m,-\frac{k-2}{2}+m}^{(s u)} .
$$

Similarly, it is sometimes useful to separate the $\mathcal{N}=2$ cigar CFT into a bosonic $S L(2) / U(1)$ theory times a scalar field. To generalize the above construction to $S L(2)_{k} / U(1)$, one simply has to take $k \rightarrow-k$ and $j \rightarrow-j-1$ in all formulae. Some of the correlators of the bosonic $S L(2) / U(1)$ theory are described in appendix B. The analog of equation (4.54) is now

$$
V_{j ; m, m}^{(s l, s u s y)}(R R,+) \simeq V_{\frac{k-2}{2}-j ; \frac{k+2}{2}+m, \frac{k+2}{2}+m}^{(s l, \text { susy })}(R R,-)
$$

It can be derived by bosonizing $\mathcal{N}=2 S L(2)_{k} / U(1)$ in terms of bosonic $S L(2) / U(1)$ and a free boson and using 63,64

$$
V_{j ; m, m}^{(s l)} \simeq V_{\frac{k-2}{2}-j ; \frac{k+2}{2}+m, \frac{k+2}{2}+m}^{(s l)}
$$

The expressions (4.56), (4.57) should be understood as statements about the normalizable states in the theory (which we will discuss in $§ 5.2$ ). For appropriate values of $j$ for which the normalizable states exist, the left-hand side of (4.56) is equal to the right-hand side up to a $j$-dependent multiplicative factor.

\subsection{Analogy to conifold}

This subsection lies somewhat outside the main line of development of the paper, and can be skipped on first reading. Its main purpose is to point out the analogy of the foregoing discussion to another familiar and well studied system - the conifold, where again, the coefficient of a term in the effective action which is protected by supersymmetry has different interpretations in different regimes.

On the geometrical side, the analogy involves replacing the ALE singularity of $K 3$, described (after deformation) by (4.2), by the deformed conifold singularity of Calabi-Yau manifolds

$$
z_{1}^{2}+z_{2}^{2}+z_{3}^{2}+z_{4}^{2}=\mu
$$


Equation (4.58) describes a conical singularity, in which a shrunken $S^{3}$ has been blown up to volume $\simeq \mu$. A $D 3$-brane wrapped around this $S^{3}$ gives a hypermultiplet of mass $M_{H} \simeq \mu / g_{s}$.

One can also realize (4.58) in terms of a T-dual NS5-brane system, by studying two $N S 5$-branes which intersect on a $3+1$-dimensional space. For example, one can take one of the NS5-branes to span the directions $\left(x^{1}, x^{2}, x^{3}, x^{4}, x^{5}\right)$, and the other to be extended in $\left(x^{1}, x^{2}, x^{3}, x^{8}, x^{9}\right)$. The intersection of the two $N S 5$-branes is the three dimensional space labeled by $\left(x^{1}, x^{2}, x^{3}\right)$. Denoting $x^{4}+i x^{5}=v, x^{8}+i x^{9}=w$, the deformation (4.58) corresponds in the $N S 5$-brane language to studying a $N S 5$-brane with worldvolume $v w=\mu$.

The analog of the $t_{8} F^{4}$ term discussed in this paper in the case of the conifold is the kinetic $\left(F^{2}\right)$ term of the RR gauge field, under which the hypermultiplet coming from the wrapped $D 3$-brane is charged. Its coefficient, which is related by $\mathcal{N}=2$ space-time supersymmetry to the metric on moduli space, goes as $\mu \rightarrow 0$ like $\log \mu$. This behavior has different interpretations in different regimes.

When the mass of the hypermultiplet $M_{H} \ll M_{s}$, one understands the $\log \mu$ as a consequence of integrating out at one-loop a light charged hypermultiplet in the low energy $\mathcal{N}=2$ Abelian gauge theory of the RR gauge field 65. This calculation can also be thought of as a one-loop calculation in the S-dual heterotic string [43].

When the mass of the hypermultiplet $M_{H} \gg M_{s}$, the same behavior arises by studying the perturbative string theory in the deformed conifold background. Similar arguments to those reviewed earlier in this section, lead in this case to the background

$$
\mathbb{R}^{3,1} \times \frac{S L(2)_{1}}{U(1)}
$$

or, equivalently, $\mathbb{R}^{3,1} \times(\mathcal{N}=2$ Liouville $)$ with superpotential $\left(Q^{2}=2 / k=2\right.$ in this case $)$

$$
W=\mu e^{-\frac{1}{\sqrt{2}} \Phi}
$$

The string coupling at the tip of the cigar is

$$
\frac{1}{g_{s}^{(\mathrm{tip})}}=\frac{M_{H}}{M_{s}} \gg 1,
$$

such that the string theory (4.59) is weakly coupled in this limit. 
The metric on moduli space is given by the two-point function of the modulus corresponding to changing $\mu$ :

$$
G=\left\langle e^{-\frac{1}{\sqrt{2}}(\phi-i Y)} e^{-\frac{1}{\sqrt{2}}(\phi+i Y)}\right\rangle .
$$

This is a bulk amplitude in the sense of Liouville theory (see e.g. [66] for a discussion). It can be computed using standard techniques, and one finds,

$$
G=-\log \left|\frac{\mu}{\Lambda}\right|,
$$

where $\Lambda \gg \mu$ is a UV cutoff, and the origin of the log is the "volume" of the Liouville direction, from the wall provided by the superpotential (4.60), or the tip of the cigar, to a cutoff $\phi_{0} \simeq \log \Lambda$ far from the wall. In other words, the origin of the $\log \mu$ behavior in this limit is in the continuum of perturbative string states living in the long throat that develops when $M_{H}$ is much smaller than $M_{\text {Planck }}$. Note in particular that the logarithmic contribution that arises at one-loop for $M_{H} \ll M_{s}$, is a tree level effect for $M_{H} \gg M_{s}$.

Clearly, the situation on the conifold is similar to the ALE case discussed here. Our case is more complicated, both because we are considering a larger class of singularities, which can be thought of as analogous to generalized conifolds,

$$
z_{1}^{k}+z_{2}^{2}+z_{3}^{2}+z_{4}^{2}=\mu,
$$

and because in six dimensions we are led to study four-point functions rather than twopoint functions (since we would like to compute $F^{4}$ terms rather than $F^{2}$ terms).

\section{Correlation function computations}

In this section we will compute the $t_{8} F^{4}$ terms in the low-energy effective action of $A_{k-1}$ LSTs in the regime $M_{W} \gg M_{s}$ discussed in $\S 4$. To do this we will study the appropriate correlation functions of the gauge-invariant operators (4.13).

As mentioned above, we are mostly interested in performing computations at a specific point in the moduli space of the LST where the VEVs of the scalar fields in the low-energy $S U(k)$ gauge theory are given by (4.29)

$$
\begin{aligned}
& \langle A\rangle=0, \\
& \langle B\rangle=r_{0} \operatorname{diag}\left(e^{2 \pi i / k}, e^{4 \pi i / k}, \cdots, e^{2 \pi i(k-1) / k}, 1\right) .
\end{aligned}
$$


Recall that we have set $M_{s}=1 / \sqrt{2}$, and that the theory is weakly coupled when $r_{0}$ is much larger than the string scale and $M_{W} \propto r_{0}$ obeys $M_{W} \gg M_{s}$. We will focus on the operators

$$
\mathcal{O}_{2 j+1}^{+}=\zeta^{\mu \nu} \widetilde{\operatorname{tr}}\left(F_{\mu \nu} B^{2 j+1}+\text { fermions } \cdot B^{2 j}\right)
$$

and their complex conjugates

$$
\mathcal{O}_{2 j+1}^{-}=\zeta^{\mu \nu} \widetilde{\operatorname{tr}}\left(F_{\mu \nu}\left(B^{*}\right)^{2 j+1}+\text { fermions } \cdot\left(B^{*}\right)^{2 j}\right)
$$

We will not be careful about the precise form of the fermionic terms in these operators since they will not contribute at the leading order in $M_{s} / M_{W} \simeq g_{s}$ to the correlation functions we will compute.

The form of the Ramond-Ramond vertex operators corresponding to the operators (5.2) with zero momentum in $\mathbb{R}^{5,1}$ in the CHS theory is given by (4.21). We will need the vertex operators at non-zero momentum $p_{\mu}$; these take the form (in the CHS theory, or in the resolved theory for large $\phi$ ) :

$$
\begin{aligned}
& \hat{\mathcal{O}}_{2 j+1}^{+}=\zeta^{\mu \nu} e^{-\frac{1}{2} \varphi-\frac{1}{2} \bar{\varphi}} \gamma_{\mu \nu}^{a \dot{a}} e^{\frac{i}{2} H+\frac{i}{2} \bar{H}} \Phi_{j ; j, j}^{(s u)} e^{Q \tilde{\jmath} \phi} e^{i p \cdot x} \\
& \left(S_{a} e^{\frac{i}{2} H^{\prime}}+\frac{i}{Q(j+\tilde{\jmath}+1)}\left(\gamma^{\rho}\right)_{a}^{\dot{c}} p_{\rho} S_{\dot{c}} e^{-\frac{i}{2} H^{\prime}}\right)\left(\bar{S}_{\dot{a}} e^{\frac{i}{2} \bar{H}^{\prime}}+\frac{i}{Q(j+\tilde{\jmath}+1)}\left(\gamma^{\sigma}\right)_{\dot{a}}^{c} p_{\sigma} \bar{S}_{c} e^{-\frac{i}{2} \bar{H}^{\prime}}\right), \\
& \hat{\mathcal{O}}_{2 j+1}^{-}=\left(\zeta^{\prime}\right)^{\mu^{\prime} \nu^{\prime}} e^{-\frac{1}{2} \varphi-\frac{1}{2} \bar{\varphi}} \gamma_{\mu^{\prime} \nu^{\prime}}^{b \dot{b}} e^{-\frac{i}{2} H-\frac{i}{2} \bar{H}} \Phi_{j ;-j,-j}^{(s u)} e^{Q \tilde{\jmath} \phi} e^{i p \cdot x} \\
& \left(S_{b} e^{-\frac{i}{2} H^{\prime}}+\frac{i}{Q(j+\tilde{\jmath}+1)}\left(\gamma^{\rho}\right)_{b}^{\dot{d}} p_{\rho} S_{\dot{d}} e^{\frac{i}{2} H^{\prime}}\right)\left(\bar{S}_{\dot{b}} e^{-\frac{i}{2} \bar{H}^{\prime}}+\frac{i}{Q(j+\tilde{\jmath}+1)}\left(\gamma^{\sigma}\right)_{\dot{b}}^{d} p_{\sigma} \bar{S}_{d} e^{\frac{i}{2} \bar{H}^{\prime}}\right),
\end{aligned}
$$

where $\tilde{\jmath}$ is the larger of the two solutions to the mass-shell condition

$$
Q^{2}(\tilde{\jmath}-j)(\tilde{\jmath}+j+1)=p^{2}
$$

$\hat{\mathcal{O}}_{2 j+1}^{-}$(with momentum $\left.\left(-p_{\mu}\right)\right)$ is the complex conjugate of $\hat{\mathcal{O}}_{2 j+1}^{+}$(with momentum $p_{\mu}$ ). We will begin in $\S 5.1$ by computing the two-point functions of these operators, in order to verify that their low-energy behavior is the same as that expected for the LST operators $\mathcal{O}_{2 j+1}^{ \pm}$(up to normalization). This will lead us to a discussion of amputated (normalizable) versions of these operators in $\S 5.2$. Then, in $\S 5.3-\S 5.5$, we will analyze the four-point functions of these operators, first in the general case and then for particular values of the $j$ 's for which the computation simplifies. 


\subsection{Two-point functions}

In order to verify the operator identifications described above, we wish to compare the two-point function of the operators (5.2), (5.3) $\left\langle\mathcal{O}_{2 j+1}^{+} \mathcal{O}_{2 j+1}^{-}\right\rangle$in the low-energy field theory to the string theory expectation value $\left\langle\hat{\mathcal{O}}_{2 j+1}^{+} \hat{\mathcal{O}}_{2 j+1}^{-}\right\rangle$, at leading order in the string coupling (or, equivalently, in $1 / r_{0}$ or in $M_{s} / M_{W}$ ). We expect that the two will be identical up to a normalization which we have not determined above, and which will be fixed by the following computations 15 .

Let us start by discussing the correlator in the low-energy field theory. The maximal power of $r_{0}$ arises if we replace all the $B$ 's in (5.2) by their VEVs (5.1) and contract the two gauge fields. The fermionic terms give rise to lower powers of $r_{0}$ so they will not be relevant. Multi-trace contributions involve factors of $\operatorname{tr}\left(\left\langle B^{l}\right\rangle\right)$ with $l<k$, which vanish at this point in moduli space. If we normalize the Abelian gauge fields in the low-energy field theory to obey $\left\langle A_{\mu}(p) A_{\nu}(-p)\right\rangle=\eta_{\mu \nu} / p^{2}$ (in Feynman gauge), we find at leading order

$$
\begin{aligned}
& \left\langle\tilde{\operatorname{tr}}\left(F_{\mu \nu} B^{2 j+1}\right)(p) \tilde{\operatorname{tr}}\left(F_{\mu^{\prime} \nu^{\prime}}\left(B^{*}\right)^{2 j+1}\right)(-p)\right\rangle= \\
& \left\langle\operatorname{tr}\left(B^{2 j+1}\left(B^{*}\right)^{2 j+1}\right)\right\rangle \frac{p_{\mu} p_{\mu^{\prime}} \eta_{\nu \nu^{\prime}} \pm\left(\mu \leftrightarrow \nu, \mu^{\prime} \leftrightarrow \nu^{\prime}\right)}{p^{2}}
\end{aligned}
$$

where $\left\langle\operatorname{tr}\left(B^{2 j+1}\left(B^{*}\right)^{2 j+1}\right)\right\rangle=k r_{0}^{2(2 j+1)}$.

We next turn to the two-point function in type IIB string theory. The operators (5.4) were written in the $(-1 / 2,-1 / 2)$-picture, so in order to compute their two-point function on the sphere we need to either shift them to another picture or add another operator in the $(-1,-1)$-picture 16 . We will use the former method. Using the fact that the picturechanging operator is given by $\left(G(z) e^{\varphi}(z)+\right.$ ghost terms $)$ and the form of $G$ from appendix A, we find that the form of $\hat{\mathcal{O}}_{2 j+1}^{+}$in the $(-3 / 2,-1 / 2),(-1 / 2,-3 / 2)$ and $(-3 / 2,-3 / 2)$

15 In fact, as shown in [54], the string theory computation described below receives also contributions that do not come from the low energy gauge theory. Fortunately, the gauge theory contribution differs from the string theory amplitude by a multiplicative $j$-independent constant (which is determined in [54]). Since in this paper we focus mostly on the $j$ dependence of the amplitudes, this will not affect our final results.

16 For example, by differentiating the two-point function with respect to the coupling $\mu$, (4.32). 
pictures is given by the following expressions 17 (at large $\phi)$ :

$$
\begin{aligned}
\hat{\mathcal{O}}_{2 j+1}^{+}= & \zeta^{\mu \nu} e^{-\frac{3}{2} \varphi-\frac{1}{2} \bar{\varphi}} \gamma_{\mu \nu}^{a \dot{a}} e^{\frac{i}{2} H+\frac{i}{2} \bar{H}} \Phi_{j ; j, j}^{(s u)} e^{Q \tilde{\jmath} \phi} e^{i p \cdot x} \cdot \\
& \left(\frac{\sqrt{2} i}{Q(j+\tilde{\jmath}+1)} S_{a} e^{-\frac{i}{2} H^{\prime}}\right)\left(\bar{S}_{\dot{a}} e^{\frac{i}{2} \bar{H}^{\prime}}+\frac{i}{Q(j+\tilde{\jmath}+1)}\left(\gamma^{\sigma}\right)_{\dot{a}}^{c} p_{\sigma} \bar{S}_{c} e^{-\frac{i}{2} \bar{H}^{\prime}}\right), \\
\hat{\mathcal{O}}_{2 j+1}^{+}= & \zeta^{\mu \nu} e^{-\frac{1}{2} \varphi-\frac{3}{2} \bar{\varphi}} \gamma_{\mu \nu}^{a \dot{a}} e^{\frac{i}{2} H+\frac{i}{2} \bar{H}} \Phi_{j ; j, j}^{(s u)} e^{Q \tilde{\jmath} \phi} e^{i p \cdot x} \cdot \\
& \left(S_{a} e^{\frac{i}{2} H^{\prime}}+\frac{i}{Q(j+\tilde{\jmath}+1)}\left(\gamma^{\rho}\right)_{a}^{\dot{c}} p_{\rho} S_{\dot{c}} e^{-\frac{i}{2} H^{\prime}}\right)\left(\frac{\sqrt{2} i}{Q(j+\tilde{\jmath}+1)} \bar{S}_{\dot{a}} e^{-\frac{i}{2} \bar{H}^{\prime}}\right), \\
\hat{\mathcal{O}}_{2 j+1}^{+}= & \zeta^{\mu \nu} e^{-\frac{3}{2} \varphi-\frac{3}{2} \bar{\varphi}} \gamma_{\mu \nu}^{a \dot{a}} e^{\frac{i}{2} H+\frac{i}{2} \bar{H}} \Phi_{j ; j, j}^{(s u)} e^{Q \tilde{\jmath} \phi} e^{i p \cdot x} \cdot \\
& \left(\frac{\sqrt{2} i}{Q(j+\tilde{\jmath}+1)} S_{a} e^{-\frac{i}{2} H^{\prime}}\right)\left(\frac{\sqrt{2} i}{Q(j+\tilde{\jmath}+1)} \bar{S}_{\dot{a}} e^{-\frac{i}{2} \bar{H}^{\prime}}\right),
\end{aligned}
$$

with $\tilde{\jmath}$ related to $j$ as in (5.5). The expressions for $\hat{\mathcal{O}}_{2 j+1}^{-}$are the complex conjugates of these. Note that the situation with Ramond-Ramond vertex operators here is different than in flat space-time. There, the $(-1 / 2,-1 / 2)$ picture vertex operator corresponds to the field strength of the RR gauge field, while the $(-1 / 2,-3 / 2)$ and $(-3 / 2,-1 / 2)$ picture vertex operators correspond to the gauge field [39]. Here, the vertex operators in all pictures correspond to the gauge-invariant operators (4.13).

Consider e.g. the two-point function of $\hat{\mathcal{O}}_{2 j+1}^{+}$, with space-time momentum $p_{\mu}$ and "Liouville momentum" $\tilde{\jmath}_{1}$, in the $(-3 / 2,-3 / 2)$ picture (last line of $\left.(5.7)\right)$ with $\hat{\mathcal{O}}_{2 j+1}^{-}$, with space-time momentum $\left(-p_{\mu}\right)$ and "Liouville momentum" $\tilde{\jmath}_{2}=\tilde{\jmath}_{1}=\tilde{\jmath}$, in the $(-1 / 2,-1 / 2)$ picture (5.4). It is useful to start by analyzing the scaling and conservation laws of this two-point function using the description of the theory as an $\mathcal{N}=2$ Liouville theory times a minimal model, as described in the previous section (4.32), 4.35).

The correlator in question scales with the Liouville coupling $\mu$ as follows:

$$
\left\langle\hat{\mathcal{O}}_{2 j+1}^{+} \hat{\mathcal{O}}_{2 j+1}^{-}\right\rangle \sim \mu^{a} \bar{\mu}^{b}
$$

The scaling exponents $a, b$ can be determined by using the symmetries. Momentum conservation in the $Y$ direction 18 leads to the condition $a=b$, since the contributions of the operators $\hat{\mathcal{O}}_{2 j+1}^{ \pm}$cancel (they have opposite $J_{3}^{\text {tot }}$ charges). By looking at the momentum in the $\phi$ direction, taking into account the background charge of this scalar on the sphere, we

17 One can write these vertex operators in other, BRST-equivalent, ways.

18 Using (4.24), and noting that $J_{3}^{\text {tot }}\left(\hat{\mathcal{O}}_{2 j+1}\right)=j+\frac{1}{2}$. 
find that $Q \tilde{\jmath}_{1}+Q \tilde{\jmath}_{2}-\frac{1}{Q}(a+b)=-Q$, or $a+b=2\left(\tilde{\jmath}_{1}+\tilde{\jmath}_{2}+1\right) / k$. At low momentum $\tilde{\jmath} \sim j$ and we get $a=b \simeq(2 j+1) / k$, meaning that the correlator scales as $(\mu \bar{\mu})^{\frac{2 j+1}{k}} \simeq r_{0}^{2(2 j+1)}$, just as we found in the field theory (5.6).

Next, we look at the momentum in the $H^{\prime}$ direction (a similar analysis holds for $\left.\bar{H}^{\prime}\right)$. Since $a=b$, the Liouville interactions do not contribute to this, and $\hat{\mathcal{O}}_{2 j+1}^{+}$in the $(-3 / 2,-3 / 2)$ picture goes like $e^{-\frac{i}{2} H^{\prime}}$, so we only get a contribution from the term scaling as $e^{\frac{i}{2} H^{\prime}}$ in the operator $\hat{\mathcal{O}}_{2 j+1}^{-}$.

Now, we are ready to compute the two-point function. As usual in theories including $S L(2) / U(1)$, we fix the positions of the two operators, and the additional zero from dividing by the volume of the conformal Killing group of the sphere with two punctures is canceled by an infinity due to integration over bosonic zero modes in the $S L(2) / U(1)$ CFT. This infinity is reflected in the factor $\delta\left(\tilde{\jmath}_{1}-\tilde{\jmath}_{2}\right)$ in the $S L(2)$ two-point function (see equation (B.19) in appendix B). The ratio of the two infinities is a $j$ dependent constant that is determined in 67 and in appendix $\mathrm{C}$.

The ghost contribution to the two-point function is one, and using the fact that $S_{a}(z) S_{\dot{a}}(w) \sim \eta_{a \dot{a}} /(z-w)^{3 / 4}$ we find that the $\mathbb{R}^{5,1}$ contribution (including both leftmovers and right-movers) is

$$
\begin{gathered}
\zeta_{\mu \nu} \zeta_{\mu^{\prime} \nu^{\prime}}^{\prime}\left(\gamma^{\mu \nu}\right)^{a \dot{a}}\left(\gamma^{\mu^{\prime} \nu^{\prime}}\right)^{b \dot{b}}\left(\frac{\sqrt{2}}{Q^{2}(j+\tilde{\jmath}+1)^{2}}\right)^{2}\left(\gamma^{\rho}\right)_{b}^{\dot{d}} p_{\rho} \eta_{a \dot{d}}\left(\gamma^{\sigma}\right)_{\dot{b}}^{d} p_{\sigma} \eta_{d \dot{a}}= \\
\zeta_{\mu \nu} \zeta_{\mu^{\prime} \nu^{\prime}}^{\prime} \frac{2}{Q^{4}(j+\tilde{\jmath}+1)^{4}} \operatorname{tr}\left(\gamma^{\mu \nu} \gamma^{\sigma} \gamma^{\mu^{\prime} \nu^{\prime}} \gamma^{\rho}\right) p_{\sigma} p_{\rho}= \\
\zeta_{\mu \nu} \zeta_{\mu^{\prime} \nu^{\prime}}^{\prime} \frac{8}{Q^{4}(j+\tilde{\jmath}+1)^{4}}\left[2\left(\eta^{\mu \mu^{\prime}} p^{\nu} p^{\nu^{\prime}} \pm\left(\mu \leftrightarrow \nu, \mu^{\prime} \leftrightarrow \nu^{\prime}\right)\right)+p_{\rho}^{2}\left(\eta^{\nu \mu^{\prime}} \eta^{\mu \nu^{\prime}}-\eta^{\mu \mu^{\prime}} \eta^{\nu \nu^{\prime}}\right)\right]
\end{gathered}
$$

The same answer arises if we make different choices for the pictures of the two operators.

To compute the "throat" contribution, we use a decomposition similar to (4.44). In the $S U(2) / U(1)$ theory we have a two-point function which is normalized to one in the conventions that we are using. We are left with a two-point function in the supersymmetric $S L(2) / U(1)$ theory, which (using the decompositions of $\S 4.3$ ) is equal to a two-point function in the bosonic $S L(2) / U(1)$ theory involving $\left\langle V_{\tilde{\jmath} ; j+1, j+1}^{(s l)} V_{\tilde{\jmath} ;-j-1,-j-1}^{(s l)}\right\rangle$, with $\tilde{\jmath}$ related to $j$ and the momentum as in (5.5). Two-point functions of this type were computed in [7] and references therein, and were found to have a pole at zero momentum 19 . At this stage

19 Additional two-point functions, which are not supposed to have poles in the low-energy field theory, were also found to have poles in [7]. We will not discuss this issue here; in this paper we will limit ourselves to operators for which this problem does not arise. 
we will find it convenient to change the normalization of the operators (5.4), (5.7), so that, as described in appendix B, they are proportional to $\tilde{V}_{\tilde{\jmath} ; j+1, j+1}$ rather than to $V_{\tilde{\jmath} ; j+1, j+1}^{(s l)}$, where $\tilde{V}$ is the $S L(2) / U(1)$ reduction of the $S L(2)$ operator $\tilde{\Phi}$ discussed in appendix B. In this normalization, and taking into account the results of appendix $\mathrm{C}$ on the normalization of two-point functions in string theory on $S L(2)$, we find the following low-momentum $(\tilde{\jmath} \rightarrow j)$ behavior 20

$$
\begin{aligned}
\left\langle\tilde{V}_{\tilde{\jmath} ; j+1, j+1} \tilde{V}_{\tilde{\jmath} ;-j-1,-j-1}\right\rangle & =\frac{1}{2 \pi^{2}}\left(\frac{2 j+1}{k}\right) \pi \frac{\Gamma(-2 \tilde{\jmath}-1) \Gamma(\tilde{\jmath}-j) \Gamma(2+j+\tilde{\jmath})}{\Gamma(2 \tilde{\jmath}+2) \Gamma(-\tilde{\jmath}-j-1) \Gamma(j+1-\tilde{\jmath})} \\
& \simeq \frac{1}{\pi}\left(\frac{2 j+1}{k}\right)^{2} \frac{1}{p^{2}} .
\end{aligned}
$$

Putting everything together, we find two terms. The second term in (5.9) gives a contribution scaling as a constant at low momentum, which is presumably non-universal and corresponds to a contact term in space-time. The first term in (5.9), together with (5.10), gives a contribution which is precisely proportional to our expected answer (5.6):

$$
\left\langle\hat{\mathcal{O}}_{2 j+1}^{+} \hat{\mathcal{O}}_{2 j+1}^{-}\right\rangle \simeq \frac{4}{\pi(2 j+1)^{2}} \zeta^{\mu \nu}\left(\zeta^{\prime}\right)^{\mu^{\prime} \nu^{\prime}} \frac{p_{\mu} p_{\mu^{\prime}} \eta_{\nu \nu^{\prime}} \pm\left(\mu \leftrightarrow \nu, \mu^{\prime} \leftrightarrow \nu^{\prime}\right)}{p^{2}} .
$$

Comparing to (5.2), (5.3), (5.6), we see that the relation between the gauge theory operators $\mathcal{O}_{2 j+1}$ and the string theory operators $\hat{\mathcal{O}}_{2 j+1}$ is (for small $p^{2}$ )

$$
\mathcal{O}_{2 j+1}^{ \pm} \leftrightarrow r_{0}^{2 j+1}(2 j+1) \sqrt{\frac{\pi k}{4}} \hat{\mathcal{O}}_{2 j+1}^{ \pm}
$$

\subsection{Amputated correlation functions and normalizable operators}

Correlation functions of non-normalizable vertex operators in holographic backgrounds correspond to correlators of local operators in the dual theory (in this case a LST). Because of the momentum dependence coming from the propagator (for example in $(5.9))$ these turn out to be somewhat complicated; this problem occurs also in other correlation functions of these operators, such as their contractions with the $t_{8} F^{4}$ vertex (2.10) discussed above. It would be nice if we could study some other class of operators

20 Here and below, in our $S L(2) / U(1)$ computations $\mu$ (4.32) is fixed to a particular value, and a particular normalization is chosen for the $S L(2) / U(1)$ operators. These choices are implicitly specified by the values of the two- and three-point functions in appendix B. As we discuss in the text, the overall power of $\mu$ in each correlation function can always be reinstated by a KPZ-type scaling analysis. 
whose correlation functions would give us directly the amputated correlators in the dual theory. Such amputated correlators are related to the S-matrix in the dual theory, which is another reason to be interested in them.

Luckily, there is a simple way to get such amputated correlation functions in holographic theories. Generally, the non-normalizable modes in such theories are related to insertions of local operators, while the normalizable modes are related to states created by these operators. In conformal theories there is a precise state/operator correspondence, but in general theories there is no such correspondence, and we expect that an operator $\mathcal{O}_{i}\left(p_{\mu}\right)$ would create (on-shell) states in the theory for (one or more) specific values of $p^{2}$. Indeed, the two-point functions of operators like the ones we discuss here were found [6, 7] to have a series of poles which may be interpreted as corresponding to such states. For the appropriate values of $p^{2}$, we expect to have normalizable operators which create these states, and the correlation functions of the normalizable modes should give us directly the S-matrix for scattering states of this type, without the "external" propagators discussed above. One way to define these amputated (normalizable) operators is by looking at the limit of the non-normalizable operators as their momentum approaches a pole in the twopoint function (which behaves as $1 /\left(p^{2}+M^{2}\right)$ ), multiplied by $\left(p^{2}+M^{2}\right)$ (in order to cancel the "external" propagator); obviously this definition only makes sense when the amputated operator is on-shell.

In the CHS background, one cannot meaningfully talk about normalizable operators, since they would necessarily be supported in the strong coupling region. This is connected with the fact, mentioned after (4.15), that naively one would have of order $k^{3}$ independent normalizable operators, while we know that when we go on the moduli space only $4(k-1)$ combinations of them make sense. To have a well-defined notion of normalizable operators, we need to resolve the singularity, e.g by the deformation (4.32). We next discuss the normalizable operators for the resolved background.

In theories like the one we are discussing here, the non-normalizable operators involve vertex operators of the type $\tilde{V}_{\tilde{\jmath} ; m, \bar{m}}$ in the bosonic $S L(2) / U(1)$ CFT (when we use the decompositions of $\S 4.3$ ), where $\tilde{j}$ is the larger root of the mass-shell condition (5.5); they behave for large $\phi$ as $e^{Q \tilde{\jmath} \phi}$. As we mentioned in $\S 4$, we expect the normalizable operators to have a similar form but with the dependence on $\phi$ being instead (for large $\phi) e^{Q \tilde{j}^{\prime} \phi}$ where $\tilde{\jmath}^{\prime}=-\tilde{\jmath}-1$ is the smaller root (instead of the larger root) of (5.5). These operators 
can be defined by studying the limit of $\tilde{V}_{\tilde{\jmath} ; m, m}$ as one approaches a pole of the two-point function. For generic $\tilde{\jmath}$, the expansion of $\tilde{V}_{\tilde{\jmath} ; m, m}$ at large $\phi$ has the form (see e.g. 58 )

$$
\tilde{V}_{\tilde{\jmath} ; m, m} \simeq a(\tilde{\jmath}, m) e^{i Q m Y}\left(e^{Q \tilde{\jmath} \phi}+C(\tilde{\jmath}, m) e^{-Q(\tilde{\jmath}+1) \phi}+\cdots\right)
$$

where $a(\tilde{\jmath}, m)$ is an overall normalization factor 21 and $C(\tilde{\jmath}, m)$ is related to the two-point function given in appendix B (equation (B.19)). As we approach a pole of the two-point function, for example at $\tilde{\jmath}=|m|-1, C(\tilde{\jmath}, m)$ blows up, and the second term in (5.13) becomes much larger than the first. We can define a normalizable operator as follows:

$$
\tilde{V}_{-|m| ; m, m} \equiv \lim _{\tilde{\jmath} \rightarrow|m|-1}(\tilde{\jmath}-|m|+1) \tilde{V}_{\tilde{\jmath} ; m, m}
$$

This operator behaves at large $\phi$ like $e^{-Q(\tilde{\jmath}+1) \phi}=e^{-Q|m| \phi}$. Thus, it is indeed normalizable; it creates from the vacuum the state with the relevant quantum numbers.

Using these normalizable operators in the bosonic $S L(2) / U(1) \mathrm{CFT}$ and the decompositions discussed in $\S 4.3$, we can construct normalizable versions of the operators (5.4). Using the decompositions (4.40), (4.44), it is easy to see that the terms in $\hat{\mathcal{O}}_{2 j+1}^{+}$scaling as $e^{\frac{i}{2} H^{\prime}}$ or $e^{\frac{i}{2} \bar{H}^{\prime}}$ in (5.4) vanish in the limit (5.14), and we are left purely with the terms scaling as $e^{-\frac{i}{2}\left(H^{\prime}+\bar{H}^{\prime}\right)}$.

The correlation functions of the normalizable versions of (5.4) constructed using (5.14) compute the amputated correlation functions in the LST, which are directly related to the S-matrix. In the next subsections we will use them to compute the $t_{8} F^{4}$ term in the lowenergy effective Lagrangian of LST. Similarly, we can construct normalizable versions of the operators (4.11), which we already used in our constructions of the deformed worldsheet Lagrangians in $\S 4.2$.

In the low-energy SYM theory, acting on the vacuum with the operators (5.2), (5.3) creates various linear combinations of single-particle states of the $(k-1)$ massless gauge bosons corresponding to the Cartan subalgebra of $S U(k)$, with coefficients obtained by replacing the $B$ 's in the definition of the operators by their VEVs. At the specific point in moduli space we are interested in, (5.1), the operators $\mathcal{O}_{2 j+1}^{+}$and $\mathcal{O}_{k-2 j-1}^{-}$create precisely the same combination of massless gauge fields (up to an overall constant). Thus, we expect that in string theory, the normalizable versions of these operators (5.4) should be the same (up to a constant).

21 This appears because we are using the operators $\tilde{V}$ rather than the operators $V^{(s l)}$ (defined in $\S 4.3)$, which have the same form with $a(\tilde{j}, m)=1$. 
Indeed, the reflection properties of the $S U(2) / U(1)$ and $S L(2) / U(1)$ vertex operators discussed in $§ 4.3$ lead to precisely such a relation. The reflection symmetries (4.54), (4.56) imply that the non-normalizable operators (in the normalization we used above) obey the relation

$$
\hat{\mathcal{O}}_{2 j+1}^{-}=\frac{k-2 j-1}{2 j+1} \hat{\mathcal{O}}_{k-2 j-1}^{+}
$$

near the massless pole in their correlation functions. This follows from the equality (up to a constant) of the corresponding normalizable operators. This provides one more check of the correspondence between the string theory and field theory analysis. At other points in the moduli space (away from the origin) there are still relations between the normalizable operators corresponding to the $\mathcal{O}^{+}$'s and $\mathcal{O}^{-}$'s, but they are more complicated than (5.15).

\subsection{Four-point functions - generalities}

In the remainder of this section we will compute the four-point function of the operators (5.4), for small energies and momenta, in type II string theory. In the next section we will compare these results with the heterotic (or low-energy field theory) computations. We will discuss only the four-point functions of the operators (5.4) which include the scalar field $B$ but not the scalar field $A$ in the low-energy field theory. Note that the operators (5.4) are already in the right picture to have a non-vanishing four-point function.

We start by analyzing the selection rules for obtaining a non-zero result and its scaling with $\mu, \bar{\mu}$. Consider the four-point function of the operators $\hat{\mathcal{O}}_{2 j_{i}+1}^{\alpha_{i}}$ (5.4) with $i=1,2,3,4$, $\alpha_{i}= \pm$. Assume that this correlator is non-zero and scales as

$$
\left\langle\prod_{i=1}^{4} \hat{\mathcal{O}}_{2 j_{i}+1}^{\alpha_{i}}\right\rangle \sim \mu^{a} \bar{\mu}^{b}
$$

First, we impose $Y$-momentum (or $J_{3}^{\text {tot }}$ charge (4.24)) conservation. The operators $\hat{\mathcal{O}}_{2 j+1}^{\alpha}$ behave as $e^{i Q \alpha(j+1 / 2) Y}$. Taking into account the $Y$-dependence of the $\mathcal{N}=2$ Liouville interaction (4.32), we have

$$
\sum_{i} Q \alpha_{i}\left(j_{i}+\frac{1}{2}\right)-\frac{1}{Q}(a-b)=0,
$$

or

$$
a-b=\sum_{i} \frac{2 j_{i}+1}{k} \alpha_{i}
$$


Next, we impose $H^{\prime}$ momentum conservation (for left or right-movers). The Liouville interaction carries charge $(a-b)$. The non-normalizable vertex operators (5.4) have two terms, one that goes like $p_{\rho}^{0} e^{i H^{\prime} \alpha_{i} / 2}$ and a second one that goes like $p_{\rho}^{1} e^{-i H^{\prime} \alpha_{i} / 2}$. If we take the $p_{\rho}^{0}$ terms from all four operators, we get the sum rule

$$
a-b+\sum_{i} \frac{\alpha_{i}}{2}=0
$$

Clearly, if all $\alpha_{i}$ have the same sign there are no solutions of (5.18) and (5.19), so this contribution to the amplitude vanishes. This is consistent with the field theory analysis, since the contraction of four operators involving $F_{\mu \nu}$ with the vertex (2.10) in free field theory actually involves eight factors of momentum $p_{\rho}$. Therefore, one expects the $t_{8} F^{4}$ term we are after to arise from the term that goes like $p_{\rho} e^{-i H^{\prime} \alpha_{i} / 2}$ in each of the four vertex operators (5.4) (times another term of the same form from the other worldsheet chirality). Using these terms, the $H^{\prime}$ sum rule takes the form

$$
a-b=\sum_{i} \frac{\alpha_{i}}{2} .
$$

Combining (5.18) and (5.20) we see that up to permutations and complex conjugation, there are three distinct possibilities for the values of $\left(\alpha_{1}, \alpha_{2}, \alpha_{3}, \alpha_{4}\right)$, and in each case we get one condition on the $j$ 's for obtaining a non-zero correlation function :

$$
\begin{array}{lll}
\left(\alpha_{1}, \alpha_{2}, \alpha_{3}, \alpha_{4}\right)=(+,+,+,+) ; & j_{1}+j_{2}+j_{3}+j_{4}=k-2 ; & a-b=2, \\
\left(\alpha_{1}, \alpha_{2}, \alpha_{3}, \alpha_{4}\right)=(+,+,+,-) ; & j_{1}+j_{2}+j_{3}-j_{4}=\frac{k-2}{2} ; & a-b=1, \\
\left(\alpha_{1}, \alpha_{2}, \alpha_{3}, \alpha_{4}\right)=(+,+,-,-) ; & j_{1}+j_{2}-j_{3}-j_{4}=0 ; & a-b=0 .
\end{array}
$$

Note that the different cases are related by the reflection property (5.15). To go from the first line to the second, one takes $j_{4} \rightarrow \frac{k-2}{2}-j_{4}$; to go from the second to the third, $j_{3} \rightarrow \frac{k-2}{2}-j_{3}$.

Finally, we can find the total power of $r_{0}$ (or $\mu$, (4.31)) associated with the four-point functions (5.16), by using KPZ scaling (in $\phi$ ), which gives rise (in the low-momentum limit) to the sum rule

$$
Q \sum_{i} j_{i}-\frac{1}{Q}(a+b)=-Q
$$

Recalling that $r_{0} \propto|\mu|^{\frac{1}{k}}$ we conclude that the four-point function scales like $r_{0}^{2 \sum_{i} j_{i}+2}$. This is in agreement with the field theory expectation. Indeed, we expect a factor of 
$M_{W}^{-2} \propto r_{0}^{-2}$ from the vertex (2.10), and a factor of $r_{0}^{2 \sum_{i} j_{i}+4}$ from the expectation value of $B$ in (5.2), (5.3). In $\S 6$ we will see that the selection rules (5.21) are also in agreement with the field theory analysis.

We still need to compute the correlation function (5.16) in cases when the selection rules (5.21) are satisfied. In order to compute the four-point function (5.16) in string theory we have to integrate over the position of one of the four vertex operators. Before performing this integration, the amplitude splits into two parts: a simple part involving the kinematic factors and the expectation value associated with the free fields on $\mathbb{R}^{5,1}$, and a non-trivial part associated with the directions transverse to the NS5-branes.

The correlation function of the $\operatorname{Spin}(5,1)$ spin fields is given by

$$
\left\langle S_{\dot{a}}(0) S_{\dot{b}}(1) S_{\dot{c}}(\infty) S_{\dot{d}}(z)\right\rangle=\epsilon_{\dot{a} \dot{b} \dot{c} \dot{d}}[z(1-z)]^{-\frac{1}{4}}
$$

Using this equation and $\gamma$ matrix identities one can show that the kinematic structure of the four-point function (5.16) at low momentum is precisely what one would obtain in field theory by contracting four operators of the form (5.2), (5.3) against the vertex (2.10). Due to the non-trivial kinematic factors one must compute the four-point function at non-zero momentum, and send the momentum to zero at the end of the calculation.

As mentioned above, the kinematic structure is complicated by the presence of the four external leg propagators. The discussion of the previous subsection suggests a nice way to simplify the calculation. By going to the poles as a function of the external momenta, and computing the residue of the poles, as in (5.14), one finds that the amputated $F^{4}$ amplitude is proportional to the four-point function of the normalizable versions of the vertex operators (5.4), which for large $\phi$ behave as

$$
\begin{aligned}
& \hat{O}_{2 j+1}^{+}=a(j, j+1) \zeta^{\mu \nu} \gamma_{\mu \nu}^{\dot{a} a} e^{-\frac{1}{2} \varphi-\frac{1}{2} \bar{\varphi}} S_{\dot{a}} \bar{S}_{a} e^{\frac{i}{2}\left(H-H^{\prime}+\bar{H}-\bar{H}^{\prime}\right)} \Phi_{j ; j, j}^{(s u)} e^{-Q(j+1) \phi} \\
& \hat{O}_{2 j+1}^{-}=a(j,-j-1) \zeta^{\mu \nu} \gamma_{\mu \nu}^{\dot{a} a} e^{-\frac{1}{2} \varphi-\frac{1}{2} \bar{\varphi}} S_{\dot{a}} \bar{S}_{a} e^{\frac{i}{2}\left(H^{\prime}-H+\bar{H}^{\prime}-\bar{H}\right)} \Phi_{j ;-j,-j}^{(s u)} e^{-Q(j+1) \phi}
\end{aligned}
$$

Here we have already taken the zero momentum limit; this limit is non-singular for the amputated amplitude. In (5.24) we chose to normalize the amputated operators such that their asymptotic form at large $\phi$ is similar to that of the non-normalizable operators but with a different exponent of $\phi$. This is not the same as the naive normalization of the amputated operators, in which we simply remove the external free field propagators from the operators (5.4). If we define "naive amputated operators" $\hat{\mathcal{O}}_{2 j+1}^{ \pm}$by just removing these external propagators, then the two-point function of such an amputated operator 
with a non-normalizable operator at $p^{2}=0$ would be the same as (5.11) but without the momentum dependence, or

$$
\left\langle\left(\hat{\mathcal{O}}_{2 j+1}^{+}\right)_{\text {amputated }} \cdot \hat{\mathcal{O}}_{2 j+1}^{-}\right\rangle=\frac{4}{\pi(2 j+1)^{2}} \zeta^{\mu \nu} \zeta_{\mu \nu}^{\prime}
$$

By following through the computation of an amputated four-point function, one can show that

$$
\left\langle\prod_{i=1}^{4}\left(\hat{\mathcal{O}}_{2 j_{i}+1}^{\alpha_{i}}\right)_{\text {amputated }}\right\rangle=\left\langle\prod_{i=1}^{4}\left(-\frac{1}{Q^{2}\left(2 j_{i}+1\right)^{2}} \cdot Q^{2}\left(2 j_{i}+1\right) \cdot \hat{O}_{2 j_{i}+1}^{\alpha_{i}}\right)\right\rangle
$$

where the first factor comes from the explicit factors in (5.4), while the second is due to (5.14) and the fact that the mass-shell condition (5.5) implies that near the pole at $\tilde{\jmath}=j$, $1 /(\tilde{\jmath}-j)=Q^{2}(2 j+1) / p^{2}$. Thus, we see that the amputated version of $\hat{\mathcal{O}}_{2 j+1}^{ \pm}$is

$$
\left(\hat{\mathcal{O}}_{2 j+1}^{ \pm}\right)_{\text {amputated }}=-\frac{1}{2 j+1} \hat{O}_{2 j+1}^{ \pm}
$$

and using (5.25) we obtain

$$
\left\langle\hat{O}_{2 j+1}^{+} \cdot \hat{\mathcal{O}}_{2 j+1}^{-}\right\rangle=-\frac{4}{\pi(2 j+1)} \zeta^{\mu \nu} \zeta_{\mu \nu}^{\prime}
$$

In fact, one can simplify the four-point function even further, by using (5.23) and the identity

$$
\zeta_{1}^{\mu_{1} \nu_{1}} \gamma_{\mu_{1} \nu_{1}}^{a_{1} a_{1}} \cdots \zeta_{4}^{\mu_{4} \nu_{4}} \gamma_{\mu_{4} \nu_{4}}^{a_{4} a_{4}} \epsilon_{\dot{a}_{1} \dot{a}_{2} \dot{a}_{3} \dot{a}_{4}} \epsilon_{a_{1} a_{2} a_{3} a_{4}}=t_{8} \zeta_{1} \zeta_{2} \zeta_{3} \zeta_{4}
$$

Roughly, this means that we can remove the $\gamma$ matrices and polarization tensors from (5.24), and the $\epsilon$ tensor from (5.23), and the resulting amplitude will compute the object that we are interested in - the coefficient of $t_{8} F^{4}$ in the vertex $(2.10)$, written in terms of the variables (5.2), (5.3). In the next subsection we will make this more precise.

\subsection{Relation to $\mathcal{N}=2$ strings}

In the previous subsection we have seen that in order to compute the coefficient of $t_{8} F^{4}$ in the low-energy effective action of LST we have to evaluate the four-point function of the normalizable operators (5.24), removing the kinematic parts which refer to $\mathbb{R}^{5,1}$.

An elegant reformulation of the problem which achieves precisely this was proposed in [9]. The key observation is that the CHS background (4.10), its resolved version (4.35), and more generally any compact or non-compact $K 3$, is a good background for the $\mathcal{N}=2$ 
string. In order to explain the utility of this observation in our context, we next briefly review some relevant aspects of $\mathcal{N}=2$ string theory (see 68 for reviews and references).

Ordinary type II string theory can be thought of as $\mathcal{N}=1$ worldsheet supergravity coupled to matter. In superconformal gauge, this means that the $\mathcal{N}=1$ superconformal group generated by the superconformal generators $G, \bar{G}$, is gauged. The $\mathcal{N}=2$ string is obtained by studying $\mathcal{N}=2$ worldsheet supergravity coupled to $\mathcal{N}=2$ supersymmetric matter. In superconformal gauge, an $\mathcal{N}=2$ superconformal group is gauged. The critical central charge is equal to six in this case; the critical dimension is four. Hence, $K 3$ is a good background of this theory.

In addition to the usual bosonic reparametrization ghosts $(c, b), \mathcal{N}=2$ string theory in superconformal gauge contains two pairs of superconformal ghosts $\left(\gamma_{1}, \beta_{1}\right)$ and $\left(\gamma_{2}, \beta_{2}\right)$ associated with the two superconformal generators $G^{-}$and $G^{+}$, respectively, and a pair of $U(1)$ ghosts $(\tilde{c}, \tilde{b})$ associated with the $U(1)$ current in the $\mathcal{N}=2$ superconformal algebra (as well as the right-moving counterparts of all these fields). The BRST current takes the form

$$
J_{B}=c T+\gamma_{1} G^{-}+\gamma_{2} G^{+}+\tilde{c} J+\cdots,
$$

where the ... stand for ghost terms that will not play a role below. We can "bosonize" the superconformal ghosts in the usual way (see e.g. [39]) by defining

$$
\partial \varphi_{j}=\beta_{j} \gamma_{j} ; \quad j=1,2
$$

and adding two $(\eta, \xi)$ systems. In [9] it was argued that the $\mathcal{N}=2$ string (or, equivalently, the $\mathcal{N}=4$ topological string) on $K 3$ computes BPS terms in the Lagrangian of the full type II string theory on $K 3 \times \mathbb{R}^{5,1}$. Our $t_{8} F^{4}$ coupling is an example of such a term; hence it should correspond to an observable in the $\mathcal{N}=2$ string. We next show that this is indeed the case.

Using formulae in appendix A, it is not difficult to verify that the following normalizable vertex operators are in the BRST cohomology of the $\mathcal{N}=2$ string in the CHS background $\mathbb{R}_{\phi} \times S U(2)_{k}$, (4.6):

$$
\begin{aligned}
& \hat{O}_{2 j+1}^{+}=a(j, j+1) e^{-\frac{1}{2}\left(\varphi_{1}+\varphi_{2}+\bar{\varphi}_{1}+\bar{\varphi}_{2}\right)+\frac{i}{2}\left(H-H^{\prime}+\bar{H}-\bar{H}^{\prime}\right)} \Phi_{j ; j, j}^{(s u)} e^{-Q(j+1) \phi}, \\
& \hat{O}_{2 j+1}^{-}=a(j,-j-1) e^{-\frac{1}{2}\left(\varphi_{1}+\varphi_{2}+\bar{\varphi}_{1}+\bar{\varphi}_{2}\right)-\frac{i}{2}\left(H-H^{\prime}+\bar{H}-\bar{H}^{\prime}\right)} \Phi_{j ;-j,-j}^{(s u)} e^{-Q(j+1) \phi} .
\end{aligned}
$$

Comparing these operators to their type II string counterparts (5.24) we see that the difference is the replacement

$$
\zeta^{\mu \nu} \gamma_{\mu \nu}^{\dot{a} a} e^{-\frac{1}{2} \varphi-\frac{1}{2} \bar{\varphi}} S_{\dot{a}} \bar{S}_{a} \rightarrow e^{-\frac{1}{2}\left(\varphi_{1}+\varphi_{2}+\bar{\varphi}_{1}+\bar{\varphi}_{2}\right)}
$$


This replacement is just what we needed above. Using equations (5.23) and (5.29) it is easy to see that the unintegrated four-point function of the operators (5.24) is equal to $t_{8} \zeta_{1} \zeta_{2} \zeta_{3} \zeta_{4}$ times the unintegrated four-point function of the operators (5.32). Therefore, the four-point function of the operators (5.32) in the $\mathcal{N}=2$ string computes precisely the quantity of interest - the coefficient of $t_{8} F^{4}$ in the effective Lagrangian of LST. This is why, by a slight abuse of notation, we have denoted these operators in the same way as (5.24). Note that, using (5.15) and (5.27), the $\mathcal{N}=2$ operators (5.32) obey the simple reflection property

$$
\hat{O}_{2 j+1}^{+}=\hat{O}_{k-2 j-1}^{-}
$$

In the next subsection we will compute the four-point function of the operators (5.32), and in the next section we will compare it with the coefficient of the $t_{8} F^{4}$ term which we computed in $\S 2$.

\subsection{Four-point functions in $\mathcal{N}=2$ string theory}

The analysis of symmetries performed in $§ 5.3$ together with the discussion of $\S 5.4$ shows that there are several types of $\mathcal{N}=2$ string correlators of the operators 5.32 that should be non-zero:

$$
\begin{array}{ll}
\left\langle\hat{O}_{2 j_{1}+1}^{+} \hat{O}_{2 j_{2}+1}^{+} \hat{O}_{2 j_{3}+1}^{+} \hat{O}_{2 j_{4}+1}^{+}\right\rangle ; & j_{1}+j_{2}+j_{3}+j_{4}=k-2, \\
\left\langle\hat{O}_{2 j_{1}+1}^{+} \hat{O}_{2 j_{2}+1}^{+} \hat{O}_{2 j_{3}+1}^{+} \hat{O}_{2 j_{4}+1}^{-}\right\rangle ; & j_{1}+j_{2}+j_{3}-j_{4}=\frac{k-2}{2}, \\
\left\langle\hat{O}_{2 j_{1}+1}^{+} \hat{O}_{2 j_{2}+1}^{+} \hat{O}_{2 j_{3}+1}^{-} \hat{O}_{2 j_{4}+1}^{-}\right\rangle ; & j_{1}+j_{2}-j_{3}-j_{4}=0,
\end{array}
$$

and the complex conjugates of these. As we mentioned after equation (5.21), it is enough to compute any one of the three classes of correlators in 5.35), since the others can then be obtained by using the reflection property (5.34).

In order to compute these correlators it is convenient to map some (or all) of the RR operators in (5.35) to the NS-NS sector. This is possible, since the spectral flow that relates these sectors is gauged in the $\mathcal{N}=2$ string. The operators that implement the spectral flow transformation can be written as 6922

$$
S^{ \pm}=e^{ \pm \frac{1}{2}\left(\varphi_{2}-\varphi_{1}\right) \pm \frac{i}{2}\left(H+H^{\prime}\right)}
$$

22 We omitted a factor $e^{ \pm \frac{1}{2} c \tilde{b}}$ in the definition of the spectral flow operators. This factor is needed for BRST invariance, but does not influence correlation functions. 
These operators have the following properties [70]:

(1) $S^{ \pm}$are in the BRST cohomology of the $\mathcal{N}=2$ string. They have dimension zero.

(2) $\partial_{z} S^{ \pm}$is BRST exact, so in correlators that contain $S^{ \pm}$and other BRST-invariant operators, one can freely move the former around.

(3) $S^{+}$and $S^{-}$generate a ring. The product of $S^{+}$and $S^{-}$is one. The other independent OPE is

$$
S^{+}(z) S^{+}(w)=e^{\varphi_{2}-\varphi_{1}+i\left(H+H^{\prime}\right)} .
$$

(4) The OPE of $S^{ \pm}$with $\hat{O}_{2 j+1}^{ \pm}$is non-singular, so we can multiply them (see below). Thus, the $\hat{O}$ 's form a module of the ring generated by $S^{+}$and $S^{-}$.

(5) The algebraic structure associated with $S^{ \pm}$is very reminiscent of the ground ring of two dimensional string theory [71]. Both are useful in constraining the dynamics.

Due to the above properties, we can insert into any correlator of BRST invariant operators the product $S^{+} S^{-}=1$ (and their anti-holomorphic counterparts) and change the positions of $S^{+}$and $S^{-}$freely without changing the answer. The following results are useful for this procedure23:

$$
\begin{aligned}
& S^{+} \bar{S}^{+} \hat{O}_{2 j+1}^{+}=e^{-\varphi_{1}-\bar{\varphi}_{1}+i(H+\bar{H})} \Phi_{j ; j, j}^{(s u)} e^{-Q(j+1) \phi}, \\
& S^{-} \bar{S}^{-} \hat{O}_{2 j+1}^{+}=e^{-\varphi_{2}-\bar{\varphi}_{2}-i\left(H^{\prime}+\bar{H}^{\prime}\right)} \Phi_{j ; j, j}^{(s u)} e^{-Q(j+1) \phi}, \\
& S^{+} \bar{S}^{+} \hat{O}_{2 j+1}^{-}=e^{-\varphi_{1}-\bar{\varphi}_{1}+i\left(H^{\prime}+\bar{H}^{\prime}\right)} \Phi_{j ;-j,-j}^{(s u)} e^{-Q(j+1) \phi}, \\
& S^{-} \bar{S}^{-} \hat{O}_{2 j+1}^{-}=e^{-\varphi_{2}-\bar{\varphi}_{2}-i(H+\bar{H})} \Phi_{j ;-j,-j}^{(s u)} e^{-Q(j+1) \phi} .
\end{aligned}
$$

Consider, for example, the four-point function on the third line of equation (5.35). By inserting $S^{+} \bar{S}^{+} S^{-} \bar{S}^{-}=1$ and acting on the $\hat{O}^{-}$'s, using (5.38), one finds (suppressing the worldsheet positions)

$$
\begin{aligned}
& \left\langle\hat{O}_{2 j_{1}+1}^{+} \hat{O}_{2 j_{2}+1}^{+} \hat{O}_{2 j_{3}+1}^{-} \hat{O}_{2 j_{4}+1}^{-}\right\rangle=\left\langle\hat{O}_{2 j_{1}+1}^{+} \hat{O}_{2 j_{2}+1}^{+} \cdot\right. \\
& \left.\quad e^{-\varphi_{1}-\bar{\varphi}_{1}+i\left(H^{\prime}+\bar{H}^{\prime}\right)} \Phi_{j_{3} ;-j_{3},-j_{3}}^{(s u)} e^{-Q\left(j_{3}+1\right) \phi} \cdot e^{-\varphi_{2}-\bar{\varphi}_{2}-i(H+\bar{H})} \Phi_{j_{4} ;-j_{4},-j_{4}}^{(s u)} e^{-Q\left(j_{4}+1\right) \phi}\right\rangle
\end{aligned}
$$

23 In these formulae, and in the ones below which involve $\Phi^{(s u)}$, we write the form of the operators in the CHS background, or in the deformed background for large $\phi$; however, the formulae should be interpreted as exact formulae in the deformed background, when we replace the CHS operators by the corresponding operators in the deformed background, as described in $\S 4.3$. 
To simplify this expression further it is useful to note that

$$
\begin{aligned}
G_{-\frac{1}{2}}^{-} \Phi_{j ; j, j}^{(s u)} e^{-Q(j+1) \phi} & =i Q(2 j+1) e^{-i H^{\prime}} \Phi_{j ; j, j}^{(s u)} e^{-Q(j+1) \phi}, \\
G_{-\frac{1}{2}}^{+} \Phi_{j ;-j,-j}^{(s u)} e^{-Q(j+1) \phi} & =i Q(2 j+1) e^{i H^{\prime}} \Phi_{j ;-j,-j}^{(s u)} e^{-Q(j+1) \phi} .
\end{aligned}
$$

Using this in (5.39) we find that

$$
\begin{aligned}
& Q^{2}\left(2 j_{3}+1\right)^{2}\left\langle\hat{O}_{2 j_{1}+1}^{+} \hat{O}_{2 j_{2}+1}^{+} \hat{O}_{2 j_{3}+1}^{-} \hat{O}_{2 j_{4}+1}^{-}\right\rangle=\left\langle\hat{O}_{2 j_{1}+1}^{+} \hat{O}_{2 j_{2}+1}^{+} \cdot\right. \\
& \left.\quad e^{-\varphi_{1}-\bar{\varphi}_{1}-\varphi_{2}-\bar{\varphi}_{2}} \Phi_{j_{3} ;-j_{3},-j_{3}}^{(s u)} e^{-Q\left(j_{3}+1\right) \phi} \cdot G_{-\frac{1}{2}}^{+} \bar{G}_{-\frac{1}{2}}^{+} e^{-i(H+\bar{H})} \Phi_{j_{4} ;-j_{4},-j_{4}}^{(s u)} e^{-Q\left(j_{4}+1\right) \phi}\right\rangle,
\end{aligned}
$$

where we used picture changing to move the operator $G_{-\frac{1}{2}}^{+} \bar{G}_{-\frac{1}{2}}^{+}$from the third to the fourth operator. Recall that our conventions are that $\varphi_{1}$ is the ghost conjugate to $G^{-}$and $\varphi_{2}$ the ghost conjugate to $G^{+}$(see (5.30)).

The fourth operator now has precisely the same form as the coupling proportional to $\bar{\lambda}_{j_{4}}$ in the worldsheet Lagrangian (4.22). Thus, taking this vertex operator to be the one which is integrated over the worldsheet, we can write

$$
\begin{aligned}
& Q^{2}\left(2 j_{3}+1\right)^{2}\left\langle\hat{O}_{2 j_{1}+1}^{+} \hat{O}_{2 j_{2}+1}^{+} \hat{O}_{2 j_{3}+1}^{-} \hat{O}_{2 j_{4}+1}^{-}\right\rangle= \\
& -\frac{\partial}{\partial \bar{\lambda}_{j_{4}}}\left\langle\hat{O}_{2 j_{1}+1}^{+} \hat{O}_{2 j_{2}+1}^{+} \cdot e^{-\varphi_{1}-\bar{\varphi}_{1}-\varphi_{2}-\bar{\varphi}_{2}} \Phi_{j_{3} ;-j_{3},-j_{3}}^{(s u)} e^{-Q\left(j_{3}+1\right) \phi}\right\rangle .
\end{aligned}
$$

If we can compute the three-point function (5.42) as a function of the couplings $\lambda_{j}$, we can obtain from it the four-point function we are after. Unfortunately, this involves solving the worldsheet CFT (4.22) at a generic point in moduli space, which seems difficult using present techniques. In order to proceed, we will specialize to the case

$$
j_{4}=\frac{k-2}{2},
$$

for which the coupling $\bar{\lambda}_{j_{4}}=\bar{\mu}$ and we can use known results for the amplitude on the right-hand side of equation (5.42) in the theory (4.35). In this case the sum rule in equation (5.35) takes the form

$$
j_{1}+j_{2}=j_{3}+\frac{k-2}{2} .
$$

We can also ignore the ghosts $\varphi_{1}$ and $\varphi_{2}$ since their correlation function is trivial. Thus, we arrive at:

$$
\begin{array}{r}
Q^{2}\left(2 j_{3}+1\right)^{2}\left\langle\hat{O}_{2 j_{1}+1}^{+} \hat{O}_{2 j_{2}+1}^{+} \hat{O}_{2 j_{3}+1}^{-} \hat{O}_{k-1}^{-}\right\rangle=-\frac{\partial}{\partial \bar{\mu}}\left\langle e^{\frac{i}{2}\left(H+\bar{H}-H^{\prime}-\bar{H}^{\prime}\right)} \Phi_{j_{1} ; j_{1}, j_{1}}^{(s u)} e^{-Q\left(j_{1}+1\right) \phi} .\right. \\
\left.e^{\frac{i}{2}\left(H+\bar{H}-H^{\prime}-\bar{H}^{\prime}\right)} \Phi_{j_{2} ; j_{2}, j_{2}}^{(s u)} e^{-Q\left(j_{2}+1\right) \phi} \cdot \Phi_{j_{3} ;-j_{3},-j_{3}}^{(s u)} e^{-Q\left(j_{3}+1\right) \phi}\right\rangle .
\end{array}
$$


The derivative with respect to $\bar{\mu}$ brings down a factor of $b / \bar{\mu}$, where the the three-point function on the right hand side of equation (5.45) goes like $\mu^{a} \bar{\mu}^{b}(5.16)$. As in the discussion after (5.16), we can calculate $a$ and $b$ by imposing the sum rules for $\phi$ charge and total $J_{3}$. In this case, using (5.44), they give rise to the constraints

$$
a+b+Q^{2}\left(2 j_{3}+\frac{k}{2}+1\right)=0
$$

and

$$
a-b+1=0
$$

Solving the two constraints one finds

$$
b=-\frac{Q^{2}}{2}\left(2 j_{3}+1\right) .
$$

What remains is to compute the three-point function (5.45) in the theory given by (4.35).

Consider first the $S U(2) / U(1)$ part of the calculation. The $S U(2) / U(1)$ component of the first operator has dimension

$$
\Delta_{1}=\frac{1}{8}+\frac{j_{1}\left(j_{1}+1\right)}{k}-\frac{\left(j_{1}+\frac{1}{2}\right)^{2}}{k}=\frac{1}{8}-\frac{1}{4 k} .
$$

So, it is a RR ground state. Its R-charge is (see $\S 4.3$ )

$$
R_{1}=\frac{1}{2}\left(1-\frac{2}{k}\right)-\frac{2}{k} j_{1}=\frac{1}{2}-\frac{2 j_{1}+1}{k} .
$$

The second operator has similar properties. For the $S U(2) / U(1)$ component of the third operator, we have

$$
\begin{aligned}
& \Delta_{3}=\frac{j_{3}\left(j_{3}+1\right)}{k}-\frac{j_{3}^{2}}{k}=\frac{j_{3}}{k}, \\
& R_{3}=\frac{2 j_{3}}{k},
\end{aligned}
$$

so it is a chiral operator. As a check, the sum of the three R-charges in (5.45) is:

$$
\frac{1}{2}-\frac{2 j_{1}+1}{k}+\frac{1}{2}-\frac{2 j_{2}+1}{k}+\frac{2 j_{3}}{k}=0,
$$

due to the sum rule (5.44).

In order to compute the $S U(2) / U(1)$ correlator, we can use the decompositions discussed in $\S 4.3$, in particular equations (4.44), (4.50). For the third operator one uses the NS-NS sector analog of these equations, whose $S U(2) / U(1)$ parts are

$$
\Phi_{j_{3} ;-j_{3},-j_{3}}^{(s u)}=e^{-i Q j_{3} Y} V_{j_{3} ;-j_{3},-j_{3}}^{(s u, s u s y)},
$$


and

$$
V_{j_{3} ;-j_{3},-j_{3}}^{(\text {su,susy }}=V_{j_{3} ;-j_{3},-j_{3}}^{(s u)} \exp \left[\frac{i\left(-2 j_{3}\right)}{\sqrt{k(k-2)}} P\right] .
$$

As a check, the $P$ charges (or $U(1)_{R}$ charges) of the three operators add up to zero, $2 j_{1}+2 j_{2}-(k-2)-2 j_{3}=0$.

The $S U(2) / U(1)$ part of (5.45) now reduces to the three-point function of the bosonic parafermion operators

$$
\left\langle V_{j_{1} ; j_{1}, j_{1}}^{(s u)} V_{j_{2} ; j_{2}, j_{2}}^{(s u)} V_{j_{3} ;-j_{3},-j_{3}}^{(s u)}\right\rangle
$$

which can be calculated using the identity (4.55) and its complex conjugate

$$
V_{j ;-m,-m}^{(s u)}=V_{\frac{k-2}{2}-j ; \frac{k-2}{2}-m, \frac{k-2}{2}-m}^{(s u)} .
$$

Applying these identities to all three operators in (5.55), we find the three-point function

$$
\left\langle V_{\frac{k-2}{2}-j_{1} ; j_{1}-\frac{k-2}{2}, j_{1}-\frac{k-2}{2}}^{(s u)} V_{\frac{k-2}{2}-j_{2} ; j_{2}-\frac{k-2}{2}, j_{2}-\frac{k-2}{2}}^{(s u)} V_{\frac{k-2}{2}-j_{3} ; \frac{k-2}{2}-j_{3}, \frac{k-2}{2}-j_{3}}^{(s u)}\right\rangle .
$$

The sum rule (5.44) implies that this $S U(2) / U(1)$ correlator can be calculated directly in $S U(2)$, since the $J_{3}$ charge is conserved. Furthermore, since this three-point function only involves components with $|m|=j$, the $S U(2)$ three-point function in question is just the structure constant that appears in appendix B,

$$
\left\langle V_{\frac{k-2}{2}-j_{1} ; j_{1}-\frac{k-2}{2}}^{(s u)} V_{\frac{k-2}{2}-j_{2} ; j_{2}-\frac{k-2}{2}}^{(s u)} V_{\frac{k-2}{2}-j_{3} ; \frac{k-2}{2}-j_{3}}^{(s u)}\right\rangle=C\left(\frac{k-2}{2}-j_{1}, \frac{k-2}{2}-j_{2}, \frac{k-2}{2}-j_{3}\right) .
$$

Using the results of [72] (reviewed in appendix B) and the sum rule (5.44), one finds

$$
C\left(\frac{k-2}{2}-j_{1}, \frac{k-2}{2}-j_{2}, \frac{k-2}{2}-j_{3}\right)=\left[\frac{\gamma\left(\frac{1}{k}\right) \gamma\left(\frac{2 j_{1}+1}{k}\right) \gamma\left(\frac{2 j_{2}+1}{k}\right)}{\gamma\left(\frac{2 j_{3}+1}{k}\right)}\right]^{\frac{1}{2}},
$$

where

$$
\gamma(x) \equiv \frac{\Gamma(x)}{\Gamma(1-x)}
$$

This completes the $S U(2) / U(1)$ part of the calculation. We next move on to the $S L(2) / U(1)$ part. As we did for the $S U(2) / U(1)$ three-point function, we will compute the $S L(2) / U(1)$ three-point function by lifting it to a three-point function in $S L(2)$.

The $S L(2) / U(1)$ part of the first operator in (5.45) is (for large $\phi$ )

$$
e^{-\frac{i}{2}\left(H^{\prime}+\bar{H}^{\prime}\right)+i Q\left(j_{1}+\frac{1}{2}\right) Y-Q\left(j_{1}+1\right) \phi} .
$$


It can be lifted to a superconformal $S L(2)$ WZW vertex operator as follows. Denote by $\tilde{\Phi}$ bosonic $S L(2)$ vertex operators (at level $k+2$, as necessary for the coset; these operators are discussed in appendix B). The supersymmetric $S L(2)$ model contains in addition three free fermions $\chi^{a}$, with $a=3,+,-$. Denote by $H^{\prime \prime}$ the scalar field that bosonizes $\chi^{ \pm}$. Now consider the vertex operator

$$
e^{-\frac{i}{2}\left(H^{\prime \prime}+\bar{H}^{\prime \prime}\right)} \tilde{\Phi}_{-j_{1}-1 ; j_{1}+1, j_{1}+1} .
$$

Using the formula for the $U(1)_{R}$ current of $S L(2) / U(1)$ written as a current in the full $S L(2)$ theory,

$$
J^{(\mathrm{sl})}=\left(1+\frac{2}{k}\right) i \partial H^{\prime \prime}+\frac{2}{k} J_{3},
$$

one finds that the $S L(2) / U(1)$ R-charge of the vertex operator (5.62) is

$$
R^{(\mathrm{sl})}=-\frac{1}{2}+\frac{2 j_{1}+1}{k} .
$$

It is easy to check that this is the same as the R-charge obtained from equation (A.19), applied to (5.61). One can check that the dimensions also agree, as well as the fact that (5.62) is a normalizable vertex operator in $S L(2)$, in agreement with its expected properties in the coset theory. Finally, the sum of the $S U(2) / U(1)$ charge (5.50) and the $S L(2) / U(1)$ charge (5.64) is zero, as expected from equation (A.21) applied to (5.45).

The second operator in (5.45) can be lifted in the same way. For the third operator, one can check that starting with the $S L(2)$ operator

$$
e^{i\left(H^{\prime \prime}+\bar{H}^{\prime \prime}\right)} \tilde{\Phi}_{-j_{3}-\frac{k}{2}-1 ;-j_{3}-\frac{k}{2}-1,-j_{3}-\frac{k}{2}-1}
$$

leads to an operator with the correct properties (dimension and R-charge) in the $S L(2) / U(1)$ theory. Note that naively this operator does not have the correct asymptotic behavior for large $\phi$ (see (5.13)), but the formulae (like (5.13)) for the asymptotic behavior can only be trusted for small $j$ and large $k$, which is never the case for (5.65). In other cases one can have operator mixings with operators having different asymptotic behaviors (with the same global symmetry charges), so it seems likely that the reduction of the operator (5.65) to the $S L(2) / U(1)$ theory would have the asymptotic behavior that we need here. 
Thus, after this lifting, we find that the $S L(2) / U(1)$ part of the correlator (5.45) is equal to the $S L(2)$ correlator

$$
\begin{aligned}
\left\langle e^{-\frac{i}{2}\left(H^{\prime \prime}+\bar{H}^{\prime \prime}\right)} \tilde{\Phi}_{-j_{1}-1 ; j_{1}+1, j_{1}+1} \cdot e^{-\frac{i}{2}\left(H^{\prime \prime}+\bar{H}^{\prime \prime}\right)} \tilde{\Phi}_{-j_{2}-1 ; j_{2}+1, j_{2}+1} \cdot\right. \\
\\
\left.e^{i\left(H^{\prime \prime}+\bar{H}^{\prime \prime}\right)} \tilde{\Phi}_{-j_{3}-\frac{k}{2}-1 ;-j_{3}-\frac{k}{2}-1,-j_{3}-\frac{k}{2}-1}\right\rangle .
\end{aligned}
$$

Note that both the $H^{\prime \prime}$ charge conservation and the $J_{3}$ sum rule are satisfied in this correlator, so there is no obstruction to calculating it directly in the $S L(2)$ WZW theory.

Essentially this calculation is done in [7], and is reviewed in appendix B. Equation (4.19) of [7] contains the residue of the poles at $|m|=j+1$ for all the external legs, which is what we need here. One has (see equation (B.25)) :

$$
\begin{aligned}
&\left\langle\tilde{\Phi}_{-j_{1}-1 ; j_{1}+1, j_{1}+1} \tilde{\Phi}_{-j_{2}-1 ; j_{2}+1, j_{2}+1} \tilde{\Phi}_{-j_{3}-\frac{k}{2}-1 ;-j_{3}-\frac{k}{2}-1,-j_{3}-\frac{k}{2}-1}\right\rangle= \\
& \frac{k \pi}{2} \sqrt{\frac{\gamma\left(1-\frac{2 j_{1}+1}{k}\right) \gamma\left(1-\frac{2 j_{2}+1}{k}\right)}{\gamma\left(\frac{1}{k}\right) \gamma\left(-\frac{2 j_{3}+1}{k}\right)}} .
\end{aligned}
$$

We are now ready to assemble all the pieces, to find the final answer for the correlator on the third line of (5.35) for the special case (5.43). We find

$$
\begin{aligned}
\left\langle\hat{O}_{2 j_{1}+1}^{+} \hat{O}_{2 j_{2}+1}^{+} \hat{O}_{2 j_{3}+1}^{-} \hat{O}_{k-1}^{-}\right\rangle \simeq \frac{1}{Q^{2}\left(2 j_{3}+1\right)^{2}} \times \frac{Q^{2}}{2}\left(2 j_{3}+1\right) \times \\
{\left[\frac{\gamma\left(\frac{1}{k}\right) \gamma\left(\frac{2 j_{1}+1}{k}\right) \gamma\left(\frac{2 j_{2}+1}{k}\right)}{\gamma\left(\frac{2 j_{3}+1}{k}\right)}\right]^{\frac{1}{2}} \times \frac{k \pi}{2}\left[\frac{\gamma\left(1-\frac{2 j_{1}+1}{k}\right) \gamma\left(1-\frac{2 j_{2}+1}{k}\right)}{\gamma\left(\frac{1}{k}\right) \gamma\left(-\frac{2 j_{3}+1}{k}\right)}\right]^{\frac{1}{2}} . }
\end{aligned}
$$

Simplifying, we find:

$$
\left\langle\hat{O}_{2 j_{1}+1}^{+} \hat{O}_{2 j_{2}+1}^{+} \hat{O}_{2 j_{3}+1}^{-} \hat{O}_{k-1}^{-}\right\rangle \simeq \text { const }
$$

where the constant appearing on the right-hand side is independent of the $j_{i}$ (and of $k$ ) 24 . Note that the result (5.69) is consistent with the reflection property (5.34) satisfied by the $\mathcal{N}=2$ string operators: we can get another amplitude of the same kind by taking, say, $j_{2} \rightarrow \frac{k-2}{2}-j_{2}$ and $j_{3} \rightarrow \frac{k-2}{2}-j_{3}$. Moreover, the reflection symmetry implies that whenever at least one of the four $j$ 's in equation (5.35) is equal to $(k-2) / 2$ or to 0 , there is no need to do any further calculations, since by using (5.34) and the answer (5.69) above we can compute the amplitude for all of these cases. The computation for other values of the $j$ 's will be left for future work.

In the next section we will show that the gauge theory result 2.10) indeed has the property predicted by the string calculation, that in the appropriate normalization the analog of (5.69) for (5.2), (5.3) is indeed independent of $j_{1}, j_{2}$ and $j_{3}$, and satisfies the selection rules $(5.35)$.

24 This constant can be computed using our techniques, but we will not attempt to fix it here. 


\section{Comparison between the type II and heterotic results}

In $\S 2$ we wrote down the form of the $t_{8} F^{4}$ coupling in the heterotic theory (2.10) in terms of the gauge fields $F^{(i)}$ of the low-energy field theory. In order to compare with the type II computations of the previous section, it is useful to rewrite this coupling in terms of the operators $\mathcal{O}_{n}^{ \pm}(5.2),(5.3)$, which appear naturally in the type II string theory. For an $S U(k)$ gauge theory (2.10) takes the form

$$
\mathcal{L}_{F^{4}}=t_{8} \sum_{l>m=0}^{k-1} \frac{1}{M^{2}\left(\alpha_{l m}\right)}\left(\vec{F} \cdot \vec{\alpha}_{l m}\right)^{4},
$$

where $M\left(\alpha_{l m}\right)$ is the mass of the W-boson corresponding to the root $\alpha_{l m}$; in the NS5brane realization this is a D-string stretched between the $l$-th and the $m$-th $N S 5$-branes. Using the form of the VEV of $B$ (5.1) one can easily compute this mass at the point in moduli space we are interested in :

$$
M\left(\alpha_{l m}\right)=r_{0}\left|\exp \left(\frac{2 \pi i}{k} l\right)-\exp \left(\frac{2 \pi i}{k} m\right)\right|=2 r_{0}\left|\sin \left(\frac{\pi}{k}(l-m)\right)\right| .
$$

In order to compare the result (6.1) to the type II calculation we should express $F$ in terms of the operators $\mathcal{O}_{n}^{+}$(we will generally suppress the space-time indices in this section). Such a replacement makes sense at leading order in $M_{s} / M_{W}$, since at this order we can simply replace $B$ in (5.2) by its expectation value, and then solve for $F$ in terms of $\mathcal{O}_{n}^{+}$, obtaining (writing the low-energy $S U(k)$ gauge field as $F_{\mu \nu}=\operatorname{diag}\left(F_{\mu \nu}^{(1)}, F_{\mu \nu}^{(2)}, \cdots, F_{\mu \nu}^{(k)}\right)$ )

$$
F^{(r)}=\frac{1}{k} \sum_{n=0}^{k-1} \frac{\mathcal{O}_{n}^{+}}{r_{0}^{n}} \exp \left(-\frac{2 \pi i}{k} r n\right)
$$

In this expression we included also an operator $\mathcal{O}_{0}^{+}$(defined like the other values of $n$ (5.2)), which vanishes due to tracelessness of $F_{\mu \nu}$, so one can choose the lower limit of the sum to be either 0 or 1 .

Now, using (6.2) and (6.3) one can express $\mathcal{L}_{F^{4}}$ of (6.1) in the following form :

$$
\mathcal{L}_{F^{4}}=t_{8} \sum_{n_{i}} \frac{4 t_{n_{1}, n_{2}, n_{3}, n_{4}}}{k^{4} r_{0}^{\sum_{i} n_{i}}} \mathcal{O}_{n_{1}}^{+} \mathcal{O}_{n_{2}}^{+} \mathcal{O}_{n_{3}}^{+} \mathcal{O}_{n_{4}}^{+}
$$

where

$$
t_{n_{1}, n_{2}, n_{3}, n_{4}}=\frac{1}{r_{0}^{2}} \sum_{l>m=0}^{k-1} \frac{e^{-\frac{\pi i}{k}(l+m) \sum n_{i}}}{\sin ^{2}\left[\frac{\pi(l-m)}{k}\right]} \prod_{i=1}^{4} \sin \left[\frac{\pi(l-m)}{k} n_{i}\right] .
$$


We chose here to write the Lagrangian using the operators $\mathcal{O}_{n}^{+}$. As discussed in $\S 5.2$, when we replace the $B$ 's in (5.2), (5.3) by their VEVs, the operators $\mathcal{O}_{n}^{+}$and $\mathcal{O}_{k-n}^{-}$are identical (up to a power of $r_{0}$ ). Thus, we can always replace (for any particular $n$ ) the operator $\mathcal{O}_{n}^{+}$ appearing in (6.4) by $\mathcal{O}_{k-n}^{-}$.

The expression (6.5) is quite complicated, so we would like to simplify it. This simplification is discussed in appendix D. The result is that

$$
t_{n_{1}, n_{2}, n_{3}, n_{4}}=\frac{k^{2}}{4 r_{0}^{2}} \min \left(n_{i}, k-n_{i}\right)
$$

if $\sum_{i=1}^{4} n_{i}=2 k$, and it vanishes otherwise.

Next, we would like to relate this result to the four-point functions that we computed in the previous section. Using equations (5.28) and (5.12) we obtain that the contraction of an amputated string theory operator $\hat{O}_{2 j+1}^{-}$with a field theory operator $\mathcal{O}_{2 j+1}^{+}$gives

$$
\left\langle\hat{O}_{2 j+1}^{-} \mathcal{O}_{2 j+1}^{+}\right\rangle=-r_{0}^{2 j+1} \sqrt{\frac{4 k}{\pi}},
$$

up to factors of momentum which can be ignored following the discussion of the previous section. The space-time Lagrangian contains in particular the vertex (6.4) with all operators at zero momentum, and we can obtain a non-zero four-point function of $\hat{O}_{2 j+1}^{-}$ operators by contracting the operators $\hat{O}_{2 j+1}^{-}$with the operators $\mathcal{O}_{2 j+1}^{+}$in the vertex (6.4). The low-energy field theory result (6.4) and equation (6.7) then imply that the four-point functions computed in the previous section should be equal to

$$
\left\langle\hat{O}_{n_{1}}^{-} \hat{O}_{n_{2}}^{-} \hat{O}_{n_{3}}^{-} \hat{O}_{n_{4}}^{-}\right\rangle=\frac{64}{\pi^{2} k^{2}} t_{n_{1}, n_{2}, n_{3}, n_{4}}
$$

Let us compare this with the results of the previous section. First, if we look at a correlation function of the form (6.8) where all $\alpha_{i}$ have the same sign, we see that the sum rule $\sum_{i} n_{i}=2 k$ required to get a non-vanishing $t_{n_{1}, n_{2}, n_{3}, n_{4}}$ is precisely the same as the sum rule we encountered in the type II computation (5.21). This is our first test of the type II/heterotic duality for four-point functions.

Next, let us make a more precise comparison with the results of $\S 5.5$. In that section we computed the correlators for which at least one of the $n_{i}$ was equal to either 1 or $k-1$. In such cases, (6.6) implies that $t_{n_{1}, n_{2}, n_{3}, n_{4}}=k^{2} / 4 r_{0}^{2}$ with no dependence on the other $n$ 's, so we expect to find

$$
\left\langle\hat{O}_{n_{1}}^{-} \hat{O}_{n_{2}}^{-} \hat{O}_{n_{3}}^{-} \hat{O}_{n_{4}}^{-}\right\rangle=\left(\frac{4}{\pi r_{0}}\right)^{2}
$$


The result (5.69) which we found in our type II computation (transformed into a correlator of four $\hat{O}^{-}$'s) has precisely this form; the two expressions have precisely the same dependence on the $n_{i}$, and as we discussed after equation (5.22) they also have the same dependence on $r_{0}$ if we reinstate the powers of $\mu$ into the $S L(2) / U(1)$ correlators, so they differ just by a constant multiplicative factor (which we did not keep track of). Thus, we find precise agreement between the four-point functions we computed in type II string theory in $\S 5$ and our heterotic (field theory) expectations. This is a strong test of type II/heterotic duality, as well as of the techniques used in studying LST.

\section{Summary and discussion of future directions}

\subsection{Summary}

Since this paper is somewhat long, it is useful to briefly summarize our main results. The bulk of the paper was devoted to an analysis of a certain half-BPS $F^{4}$ term in the low-energy effective action of LST. We showed that this term can be efficiently computed in a topological version of LST, which is holographically dual to the $\mathcal{N}=2$ string on an asymptotically linear-dilaton background. Its coefficient is given by a tree level four-point function of certain normalizable operators in the $\mathcal{N}=2$ string.

At the same time, this term can be obtained by a one-loop calculation in the heterotic string on $T^{4}$ near a point of enhanced ADE gauge symmetry, or equivalently in the lowenergy SYM theory corresponding to the relevant ADE gauge group. The $\mathcal{N}=2$ string and heterotic computations of the $F^{4}$ term are apriori valid in different regions in moduli space. The expected agreement between them is a highly non-trivial consequence of heterotic/type II duality, and of the non-renormalization theorem of this $F^{4}$ term in the effective action. Therefore, computing it in the asymptotically linear dilaton background of the $\mathcal{N}=2$ string provides a sensitive test of both S-duality and the non-renormalization theorem, and it also tests our understanding of LST at low energies.

While the heterotic one-loop calculation can be easily done for all values of the moduli of LST, the $\mathcal{N}=2$ string calculation simplifies at a specific point in moduli space, (5.1). We found that a large class of four-point functions in the $\mathcal{N}=2$ string agree with the heterotic predictions at this point in moduli space.

Our results passed several consistency checks:

(1) The holographic interpretation of physics in linear dilaton spaces [3] suggests that while in the heterotic string one can consider the gauge field strength $F$ directly, 
in the type II and $\mathcal{N}=2$ string calculations, the observables correspond to gauge invariant operators in the full non-abelian gauge theory, such as $\mathcal{O}_{n}^{ \pm},(5.2),(5.3)$. We indeed found that in order to compare the linear dilaton results to those obtained in the heterotic string or low-energy SYM theory, one has to perform the discrete Fourier transform (6.3).

(2) The low-energy field theory analysis suggests that the normalizable versions of the two types of operators that we analyzed, $\mathcal{O}_{n}^{+}$and $\mathcal{O}_{k-n}^{-}$, should be the same (up to a constant), and we found that this is indeed true (both in the type II string and in the $\mathcal{N}=2$ string). In fact, the gauge theory provides a physical interpretation of certain reflection symmetries of two dimensional CFTs such as the parafermion theory and $\mathcal{N}=2$ minimal models, (4.54), (4.55), (and their $S L(2)$ analogs) that are known for many years.

(3) Expressing the $t_{8} F^{4}$ term (found from the heterotic computation) in the basis of the operators $\mathcal{O}_{n}^{ \pm}$, we found that the resulting four-point functions should obey specific selection rules. The same selection rules appeared in the type II computation in a completely different way (described in $§ 5.3$ ).

(4) We explicitly computed using the $\mathcal{N}=2$ string specific four-point functions (related to $t_{8} F^{4}$ terms) involving at least one operator $\mathcal{O}_{2 j+1}^{ \pm}$with $j=0$ or $j=(k-2) / 2$, and found that they agreed (up to an overall constant that we did not determine) with the heterotic computation of the same objects.

In the process of computing the $t_{8} F^{4}$ terms we clarified some additional issues in the study of LSTs. We showed that one could use normalizable vertex operators (which we carefully defined in §5.2) to compute amputated correlation functions in LSTs (the same should be true for other holographic backgrounds). This implements the LSZ reduction in the "boundary theory" directly in terms of the bulk variables and, as we have seen, simplifies the computation of S-matrix elements and coefficients of terms in the effective Lagrangian in these theories. Also, in order to complete our computation we needed to carefully compute the two-point functions in string theory on $S L(2) / U(1)$. As in Liouville theory or $S L(2)$, this computation naively involves a ratio of infinities, but we showed (in appendix $\mathrm{C}$ ) that the result can be uniquely determined by relating the two-point functions to three-point functions. These results are useful for many other computations in LST and in other holographic backgrounds.

We also showed that the correspondence between the asymptotically linear dilaton background (4.35) and LST sheds new light on the equivalence between $\mathcal{N}=2$ Liouville 
theory and the cigar $(S L(2) / U(1))$ CFT, conjectured in [6] and further discussed in [5759. It suggests a picture, compatible with the worldsheet analysis, according to which the black hole metric and the $\mathcal{N}=2$ superpotential (4.32) coexist in these backgrounds. In fact, our discussion of the normalizable operators in $\S 5.2$, applied to the moduli, shows that for any finite value of $\mu$, the normalizable operators corresponding to the Liouville deformation (4.32) and to the cigar deformation (4.34) should be identified (in analogy with our identification (5.15) ). There is only a single deformation operator corresponding to moving along the flat direction (5.1), though we can describe it in two (and, in fact, more) different ways. This provides additional evidence for the equivalence of the two theories.

One of our main results was the identification of some of the observables in the topological LST (holographically described by the $\mathcal{N}=2$ string on the "throat" background). We found that these observables correspond to normalizable versions of the vertex operators of the LST. Their correlation functions compute amputated correlation functions in the LST, which are related to protected terms in the effective action. Due to the spectral flow properties of $\mathcal{N}=2$ strings, the same observables correspond in the type II string both to RR operators and to NS-NS operators (the moduli of the "throat" background). We computed several three-point and four-point functions of these observables, and found that both can be non-zero. By differentiating with respect to the couplings $\lambda_{j}$ (4.27) one finds non-zero higher point functions as well. Note that this is different from the case of $\mathcal{N}=2$ strings in flat space, where all four and higher point functions vanish.

\subsection{Discussion of future directions}

There are several future directions that are suggested by our results. The most obvious is to complete the analysis of $t_{8} F^{4}$ terms. In this paper we only computed a subset of the relevant four-point functions in the topological LST, at a particular point in the moduli space (4.30). It would be nice to compute the rest of the four-point functions at this point in the moduli space, and then understand the structure everywhere in moduli space.

From the heterotic side we have predictions (6.6), 6.8) for all four-point functions of the operators (5.32) at the point (4.30) in moduli space. We show in appendix D that the heterotic answer can be naturally expressed using the fusion coefficients of the $S U(2)_{k-2}$ WZW theory. This is very suggestive, since at this point in moduli space, the relevant bulk background involves precisely this current algebra (see 4.35)). It would be interesting to understand this relation to $S U(2)_{k-2}$ better. 
More generally, the heterotic result (2.10) predicts the correlation functions of the normalizable topological observables (5.32) everywhere in moduli space. It would be interesting to verify these predictions using $\mathcal{N}=2$ strings on the generic "throat geometries" (4.27). The techniques developed in previous studies of topological string theories may be useful for this (see [73] for a review).

It should also be interesting to generalize our results to other LSTs. We expect the generalization to $D_{k}$ and $E_{k}$ type LSTs to be straightforward. It may be possible to generalize the results also to six dimensional LSTs with $\mathcal{N}=(2,0)$ supersymmetry, which arise in type IIB string theory near ALE singularities. In this case the low-energy effective action contains two-form gauge potentials rather than one-forms, but presumably there are still protected $H^{4}$ terms in the low-energy effective action that may be computable using topological strings. It is not clear if there is any useful dual description in this case. Another interesting generalization is to the topological sectors of the $3+1$ dimensional LSTs that are associated to generalized conifold-type singularities (e.g. (4.64)). The holographic dual of these topological LSTs involves $\mathcal{N}=2$ topological strings on "throat" backgrounds similar to those that we described in this paper. As we discussed in $\S 4.4$, in this case the analog of the $t_{8} F^{4}$ term studied here is a tree level two-point function in the topological string theory.

Our computations in this paper focused on three-point and four-point functions at tree level in the $\mathcal{N}=2$ string on the "throat" background. Clearly, there are many other objects that can be computed in this theory. Our analysis of $\S 5.5$ showed that the fourpoint functions in this theory were derivatives of three-point functions with respect to the moduli, and one can similarly show that they are second derivatives of two-point functions with respect to the moduli. So, the full information about tree level correlation functions seems to be contained in the two-point functions (as a function of the moduli). One should be able to define a "sphere partition function" such that the four-point functions would be its fourth derivatives. Higher genus partition functions in this theory are related [9] to $R^{4} F^{4 g-4}$ terms in the effective action. We hope that such terms may again be computed using the duality to heterotic strings, and perhaps they can also be computed directly in the $\mathcal{N}=2$ string.

As we mentioned in the introduction, one of our motivations for studying these topological LSTs is the hope that they can be a useful toy model for studying dualities between open and closed strings, like those that were found for $\mathcal{N}=2$ topological strings on conifold backgrounds. We expect that these dualities should be similar to those of $D \leq 2$ 
dimensional bosonic and fermionic strings, where the closed string theory on the throat background is equivalent to an open string theory living on D-branes localized inside the "throat"25. For the case of the $A_{1}$ ALE space this conjecture was first made in [74]. We hope that our results, and in particular the identification of the observables of the topological LST, will be useful for understanding this duality better; we hope to return to it in future work. Recent results on closed and open $\mathcal{N}=2$ strings [75-77] should be useful for the further study of $\mathcal{N}=2$ strings on ALE spaces.

\section{Acknowledgements}

We would like to thank M. Berkooz, A. Giveon, E. Kiritsis, O. Lechtenfeld, Y. Oz, B. Pioline, O. Ruchayskiy, S. Sethi, S. Stieberger, P. Vanhove and E. Verlinde for useful discussions. OA would like to thank Stanford University, SLAC, Harvard University, the University of Chicago, the Aspen Center for Physics, and the University of British Columbia for hospitality during the work on this project. BF would like to thank the University of California at Santa Cruz, Stanford University, Rutgers University, and École Normale Supérieure (Paris) for hospitality. DK thanks the Weizmann Institute, Rutgers University, LPT at École Normale Supérieure (Paris), LPTHE at Université Paris VI, and the Aspen Center for Physics for hospitality. The work of OA and BF was supported in part by the Israel-U.S. Binational Science Foundation, by the ISF Centers of Excellence program, by the European network HPRN-CT-2000-00122, and by Minerva. OA is the incumbent of the Joseph and Celia Reskin career development chair. The work of BF is also supported by an European Community Marie Curie Fellowship. The work of DK and DS is supported in part by DOE grant \#DE-FG02-90ER40560.

\section{Appendix A. Some results on CFT in the CHS background}

The CHS CFT contains a scalar $\phi$, a bosonic $S U(2)$ WZW model at level $k-2(k$ is the number of $N S 5$-branes) with currents $J^{i}, i=1,2,3$, and four fermions $\psi_{\phi}, \psi_{1}, \psi_{2}, \psi_{3}$.

25 The backgrounds discussed in this paper are very similar to the $c<1$ and $\hat{c}<1$ string theories. If one replaces the $\mathcal{N}=0(1)$ minimal model by an $\mathcal{N}=2$ minimal model, and $\mathcal{N}=0(1)$ worldsheet gravity by $\mathcal{N}=2$ worldsheet gravity, one goes from the well understood examples related to the "old matrix models" to our system. 
The operator product expansions (OPEs) are (we take $\alpha^{\prime}=2$ throughout this appendix):

$$
\begin{aligned}
\phi(z) \phi(0) & =-\log |z|^{2}, \\
\psi_{a}(z) \psi_{b}(0) & =\frac{\delta_{a b}}{z} \\
J^{i}(z) J^{j}(0) & =\frac{\frac{1}{2}(k-2) \delta^{i j}}{z^{2}}+i \epsilon^{i j k} \frac{J^{k}(0)}{z},
\end{aligned}
$$

where $\epsilon^{123}=1 ; a, b=\phi, 1,2,3$. Define

$$
J^{ \pm}=J^{1} \pm i J^{2}
$$

The OPE algebra on the last line of (A.1) is:

$$
\begin{aligned}
J^{3}(z) J^{3}(0) & =\frac{\frac{1}{2}(k-2)}{z^{2}}, \\
J^{3}(z) J^{ \pm}(0) & =\frac{ \pm J^{ \pm}(0)}{z} \\
J^{+}(z) J^{-}(0) & =\frac{k-2}{z^{2}}+\frac{2 J^{3}(0)}{z} .
\end{aligned}
$$

Also define

$$
\begin{aligned}
\psi^{ \pm} & =\frac{1}{\sqrt{2}}\left(\psi_{1} \pm i \psi_{2}\right), \\
\psi & =\frac{1}{\sqrt{2}}\left(\psi_{\phi}+i \psi_{3}\right),
\end{aligned}
$$

which satisfy

$$
\psi(z) \psi^{*}(0)=\psi^{+}(z) \psi^{-}(0)=\frac{1}{z} .
$$

Sometimes it is convenient to bosonize the fermions (A.4) and write them as

$$
\psi^{ \pm}=e^{ \pm i H} ; \quad \psi=e^{i H^{\prime}}
$$

As mentioned in $\S 4$, the total $S U(2)$ currents of the supersymmetric $S U(2)_{k} \mathrm{CFT}$, of level $k$, receive a contribution also from the fermions. They are given by

$$
J_{i}^{\text {tot }}=J_{i}-\frac{i}{2} \epsilon_{i j k} \psi_{j} \psi_{k}
$$

and in particular

$$
J_{3}^{\text {tot }}=J_{3}-i \psi_{1} \psi_{2}=J_{3}+\psi^{+} \psi^{-} .
$$

Note in particular that $\psi^{ \pm}$have charge \pm 1 under $J_{3}^{\text {tot }}$. 
The stress tensor of the model is given by

$$
T=-\frac{1}{2}(\partial \phi)^{2}-\frac{1}{2} Q \partial^{2} \phi+\frac{1}{k+2} J_{i} J_{i}-\frac{1}{2} \psi^{*} \partial \psi-\frac{1}{2} \psi \partial \psi^{*}-\frac{1}{2} \psi^{+} \partial \psi^{-}-\frac{1}{2} \psi^{-} \partial \psi^{+},
$$

where for $\alpha^{\prime}=2$ the slope of the linear dilaton is (see (4.7)) the positive root of

$$
Q^{2}=\frac{2}{k}
$$

In particular, (A.9) implies that the dimension of $e^{\beta \phi}$ is

$$
\Delta\left(e^{\beta \phi}\right)=-\frac{1}{2} \beta(\beta+Q) .
$$

The CHS CFT has an $\mathcal{N}=4$ superconformal symmetry. For our discussion it is useful to exhibit an $\mathcal{N}=2$ subalgebra of it. The two superconformal generators are (see [78]) :

$$
\begin{gathered}
G=i \psi_{\phi} \partial \phi+i Q \partial \psi_{0}+Q\left(J_{1} \psi_{1}+J_{2} \psi_{2}+J_{3} \psi_{3}-i \psi_{1} \psi_{2} \psi_{3}\right), \\
G_{3}=i \psi_{3} \partial \phi+i Q \partial \psi_{3}+Q\left(-J_{3} \psi_{\phi}+J_{1} \psi_{2}-J_{2} \psi_{1}+i \psi_{1} \psi_{2} \psi_{\phi}\right) .
\end{gathered}
$$

One can define the generators

$$
G^{ \pm}=G \pm i G_{3}
$$

which are given by

$$
\begin{aligned}
& G^{+}=i \psi\left(\partial \phi-Q J_{3}^{\mathrm{tot}}\right)+i Q \partial \psi+Q J^{-} \psi^{+}, \\
& G^{-}=i \psi^{*}\left(\partial \phi+Q J_{3}^{\mathrm{tot}}\right)+i Q \partial \psi^{*}+Q J^{+} \psi^{-} .
\end{aligned}
$$

These generators satisfy the OPE algebra

$$
G^{+}(z) G^{-}(0) \simeq \frac{2 c}{3 z^{3}}+\frac{2}{z^{2}} J(0)+\frac{1}{z}(2 T(0)+\partial J(0)),
$$

from which one can find the form of the $U(1)_{R}$ current $J$. In computing the OPE (A.16), one notes that $G^{+}$and $G^{-}$split into two decoupled terms: the first two terms in each line in (A.15), and the last term. Thus, in computing the OPE (A.16) we can separate:

$$
\begin{aligned}
G^{+}(z) G^{-}(0)=- & {\left[\psi\left(\partial \phi-Q J_{3}^{\mathrm{tot}}\right)+Q \partial \psi\right](z)\left[\psi^{*}\left(\partial \phi+Q J_{3}^{\mathrm{tot}}\right)+Q \partial \psi^{*}\right](0)+} \\
& Q^{2} J^{-} \psi^{+}(z) J^{+} \psi^{-}(0) .
\end{aligned}
$$

The term that goes like $1 / z^{3}$ is

$$
(-1)\left(-1-Q^{2} \frac{k}{2}\right)-Q^{2}(-2)+Q^{2}(k-2)=2+\frac{4}{k}+\frac{2}{k}(k-2)=4=\frac{2}{3} c,
$$


in agreement with (A.16).

The coefficient of $1 / z^{2}$ should be $2 J$, the $U(1)_{R}$ current in the $\mathcal{N}=2$ superconformal algebra. From the square bracket term in (A.17) we get

$$
2 J_{s l}=2 \psi \psi^{*}+2 Q^{2} J_{3}^{\text {tot }} .
$$

From the last term in (A.17):

$$
2 J_{s u}=Q^{2}(k-2) \psi^{+} \psi^{-}+Q^{2}(-2) J_{3} .
$$

After resolving the strong coupling singularity as discussed in section 4, (A.19) and (A.20) become the $U(1)_{R}$ currents of the $S L(2) / U(1)$ and $S U(2) / U(1)$ SCFTs, respectively. The sum of (A.19) and (A.20) is $2 J$ with

$$
J=\psi \psi^{*}+\psi^{+} \psi^{-} .
$$

\section{Appendix B. Some facts about $S U(2)$ and $S L(2)$ WZW models}

In this appendix we review some properties of the $S U(2)$ and $S L(2)$ WZW models which are used in the main part of this paper.

The $S U(2)$ WZW modele 26 of level

$$
k_{S U(2)}=k-2,
$$

has the central charge

$$
c_{S U(2)}=\frac{3(k-2)}{k}
$$

and contains an $S U(2)_{L} \times S U(2)_{R}$ affine Lie algebra of level $k_{S U(2)}$. The operator algebra consists of primary fields of the current algebra $\Phi_{j}^{(s u)}$ with $j=0,1 / 2, \cdots,(k-2) / 2$ and their descendants. The primaries $\Phi_{j}^{(s u)}$ can be written in two equivalent bases [72], which are related by

$$
\Phi_{j}^{(s u)}(y, \bar{y} ; z, \bar{z})=\sum_{m, \bar{m}=-j}^{j}\left[C_{2 j}^{m+j} C_{2 j}^{\bar{m}+j}\right]^{\frac{1}{2}} y^{j+m} \bar{y}^{j+\bar{m}} \Phi_{j ; m, \bar{m}}^{(s u)}(z, \bar{z}),
$$

26 For more details and conventions see 772 . 
where $C_{N}^{m}=\frac{N !}{m !(N-m) !}$ are binomial coefficients and the $\Phi_{j ; m, \bar{m}}^{(s u)}$ are eigenfunctions of $J_{3}$ and $\bar{J}_{3}$ with eigenvalues $m$ and $\bar{m}$.

The form of two- and three-point functions of the operators is fixed by $S U(2)$ invariance. In particular, the two-point functions of primaries are determined up to an overall $j$-dependent factor, which can be chosen to be one,

$$
\begin{aligned}
& \left\langle\Phi_{j_{1}}^{(s u)}\left(y_{1}, \bar{y}_{1}\right) \Phi_{j_{2}}^{(s u)}\left(y_{2}, \bar{y}_{2}\right)\right\rangle=\delta_{j_{1}, j_{2}}\left|y_{12}\right|^{4 j_{1}}, \\
& \left\langle\Phi_{j_{1} ; m_{1}, \bar{m}_{1}}^{(s u) \dagger} \Phi_{j_{2} ; m_{2}, \bar{m}_{2}}^{(s u)}\right\rangle=\delta_{j_{1}, j_{2}} \delta_{m_{1}, m_{2}} \delta_{\bar{m}_{1}, \bar{m}_{2}},
\end{aligned}
$$

where $y_{i j} \equiv y_{i}-y_{j}$ and

$$
\Phi_{j, m, \bar{m}}^{(s u) \dagger}=(-1)^{2 j-m-\bar{m}} \Phi_{j,-m,-\bar{m}}^{(s u)} .
$$

Here and below we suppress the $z$ dependence which is determined by conformal invariance. Similarly, the three-point functions of primary fields have the form

$$
\begin{aligned}
\left\langle\Phi_{j_{1}}^{(s u)}\left(y_{1}, \bar{y}_{1}\right) \Phi_{j_{2}}^{(s u)}\left(y_{2}, \bar{y}_{2}\right)\right. & \left.\Phi_{j_{3}}^{(s u)}\left(y_{3}, \bar{y}_{3}\right)\right\rangle= \\
& C\left(j_{1}, j_{2}, j_{3}\right)\left|y_{12}\right|^{2\left(j_{1}+j_{2}-j_{3}\right)}\left|y_{13}\right|^{2\left(j_{1}+j_{3}-j_{2}\right)}\left|y_{23}\right|^{2\left(j_{2}+j_{3}-j_{1}\right)} .
\end{aligned}
$$

$C\left(j_{1}, j_{2}, j_{3}\right)$ is uniquely determined once we fix the normalization of the operators using (B.4). One finds 72

$$
C^{2}\left(j_{1}, j_{2}, j_{3}\right)=\gamma\left(\frac{1}{k}\right) P^{2}\left(j_{1}+j_{2}+j_{3}+1\right) \prod_{n=1}^{3} \gamma\left(1-\frac{2 j_{n}+1}{k}\right) \frac{P^{2}\left(j_{1}+j_{2}+j_{3}-2 j_{n}\right)}{P^{2}\left(2 j_{n}\right)},
$$

where $\gamma(x)$ and $P(j)$ are defined as follows :

$$
\gamma(x) \equiv \frac{\Gamma(x)}{\Gamma(1-x)}
$$

and

$$
P(j) \equiv \prod_{n=1}^{j} \gamma\left(\frac{n}{k}\right), \quad P(0) \equiv 1
$$

One can translate $(\overline{\mathrm{B} .6})$ into the $(j, m, \bar{m})$ basis using equation $(\overline{\mathrm{B} .3})$. For the special case $j_{3}=j_{1}+j_{2}, m_{2}=\bar{m}_{2}=-j_{2}, m_{3}=\bar{m}_{3}=j_{3}$ that is used in $\S 5$ we find, using (B.3) and (B.6),

$$
\left\langle\Phi_{j_{1} ;-j_{1},-j_{1}}^{(s u)} \Phi_{j_{2} ;-j_{2},-j_{2}}^{(s u)} \Phi_{j_{1}+j_{2} ; j_{1}+j_{2}, j_{1}+j_{2}}^{(s u)}\right\rangle=C\left(j_{1}, j_{2}, j_{1}+j_{2}\right)
$$

where

$$
C^{2}\left(j_{1}, j_{2}, j_{1}+j_{2}\right)=\gamma\left(\frac{1}{k}\right) \gamma\left(1-\frac{2 j_{1}+1}{k}\right) \gamma\left(1-\frac{2 j_{2}+1}{k}\right) \gamma\left(\frac{2\left(j_{1}+j_{2}\right)+1}{k}\right) .
$$


We next turn to a discussion of the $S L(2)$ WZW model of level

$$
k_{S L(2)}=k+2
$$

with central charge

$$
c_{S L(2)}=\frac{3(k+2)}{k} .
$$

The natural observables in the theory defined on the Euclidean version of $S L(2)$, $H_{3}^{+} \equiv S L(2, \mathbb{C}) / S U(2)$, are primaries $\Phi_{j}^{(s l)}(x, \bar{x})$ of the $S L(2)_{L} \times S L(2)_{R}$ current algebra [79,80] with $j>-\frac{1}{2}$. The worldsheet scaling dimension of $\Phi_{j}^{(s l)}(x, \bar{x})$ is

$$
\Delta(j)=-\frac{j(j+1)}{k}
$$

In the papers 79,80 the operators $\Phi_{j}^{(s l)}$ are normalized as follows:

$$
\left\langle\Phi_{j_{1}}^{(s l)}\left(x_{1}, \bar{x}_{1}\right) \Phi_{j_{2}}^{(s l)}\left(x_{2}, \bar{x}_{2}\right)\right\rangle=\delta\left(j_{1}-j_{2}\right) \frac{k}{\pi}\left[\frac{1}{k \pi} \gamma\left(\frac{1}{k}\right)\right]^{2 j_{1}+1} \gamma\left(1-\frac{2 j_{1}+1}{k}\right)\left|x_{12}\right|^{-4\left(j_{1}+1\right)} .
$$

For our computations in section 5 it is more convenient to choose a different normalization

$$
\tilde{\Phi}_{j}(x, \bar{x}) \equiv \frac{\Phi_{j}^{(s l)}(x, \bar{x})}{\sqrt{\frac{k}{\pi}\left[\frac{1}{k \pi} \gamma\left(\frac{1}{k}\right)\right]^{2 j+1} \gamma\left(1-\frac{2 j_{1}+1}{k}\right)}}
$$

In this normalization the two-point function is (compare to (B.4))

$$
\left\langle\tilde{\Phi}_{j_{1}}\left(x_{1}, \bar{x}_{1}\right) \tilde{\Phi}_{j_{2}}\left(x_{2}, \bar{x}_{2}\right)\right\rangle=\delta\left(j_{1}-j_{2}\right)\left|x_{12}\right|^{-4\left(j_{1}+1\right)}
$$

For discussing the coset $S L(2) / U(1)$ it is convenient to choose a different basis for the primaries $\tilde{\Phi}_{j}$

$$
\tilde{\Phi}_{j ; m, \bar{m}}=\int d^{2} x x^{j+m} \bar{x}^{j+\bar{m}} \tilde{\Phi}_{j}(x, \bar{x}),
$$

which is analogous to the $(j ; m, \bar{m})$ basis of $S U(2)$ WZW (B.3). The two-point function in this basis was computed in [81,7] :

$$
\left\langle\tilde{\Phi}_{j ; m, \bar{m}} \tilde{\Phi}_{j^{\prime} ;-m,-\bar{m}}\right\rangle=\pi \delta\left(j-j^{\prime}\right) \frac{\Gamma(-2 j-1) \Gamma(j-m+1) \Gamma(1+j+\bar{m})}{\Gamma(2 j+2) \Gamma(-j-m) \Gamma(\bar{m}-j)} .
$$

The three-point function takes the form

$$
\begin{aligned}
& \left\langle\tilde{\Phi}_{j_{1}}\left(x_{1}, \bar{x}_{1}\right) \tilde{\Phi}_{j_{2}}\left(x_{2}, \bar{x}_{2}\right) \tilde{\Phi}_{j_{3}}\left(x_{3}, \bar{x}_{3}\right)\right\rangle= \\
& \tilde{D}\left(j_{1}, j_{2}, j_{3}\right)\left|x_{12}\right|^{2\left(j_{3}-j_{1}-j_{2}-1\right)}\left|x_{13}\right|^{2\left(j_{2}-j_{1}-j_{3}-1\right)}\left|x_{23}\right|^{2\left(j_{1}-j_{2}-j_{3}-1\right)}
\end{aligned}
$$


where the structure constants $\tilde{D}\left(j_{1}, j_{2}, j_{3}\right)$ were computed in 779,80 :

$$
\begin{aligned}
& \tilde{D}\left(j_{1}, j_{2}, j_{3}\right)=\frac{1}{2 \pi} \frac{1}{\sqrt{\gamma\left(\frac{1}{k}\right) \prod_{i=1}^{3} \gamma\left(1-\frac{2 j_{i}+1}{k}\right)}} \times \\
& \quad \frac{G\left(-j_{1}-j_{2}-j_{3}-2\right) G\left(j_{3}-j_{1}-j_{2}-1\right) G\left(j_{2}-j_{1}-j_{3}-1\right) G\left(j_{1}-j_{2}-j_{3}-1\right)}{G(-1) G\left(-2 j_{1}-1\right) G\left(-2 j_{2}-1\right) G\left(-2 j_{3}-1\right)} .
\end{aligned}
$$

$G(j)$ is a special function which satisfies the following useful identities :

$$
\begin{aligned}
G(j) & =G(-j-1-k), \\
G(j-1) & =\gamma\left(1+\frac{j}{k}\right) G(j), \\
G(j-k) & =k^{-(2 j+1)} \gamma(j+1) G(j) .
\end{aligned}
$$

In the $j, m, \bar{m}$ basis the three-point function for $m=\bar{m}$ is given by

$$
\begin{aligned}
& \left\langle\tilde{\Phi}_{j_{1} ; m_{1}, m_{1}} \tilde{\Phi}_{j_{2} ; m_{2}, m_{2}} \tilde{\Phi}_{j_{3} ; m_{3}, m_{3}}\right\rangle=\tilde{D}\left(j_{1}, j_{2}, j_{3}\right) \times \\
& F\left(j_{1}, m_{1} ; j_{2}, m_{2} ; j_{3}, m_{3}\right) \int d^{2} x|x|^{2\left(m_{1}+m_{2}+m_{3}-1\right)},
\end{aligned}
$$

where

$$
\begin{aligned}
& F\left(j_{1}, m_{1} ; j_{2}, m_{2} ; j_{3}, m_{3}\right)=\int d^{2} x_{1} d^{2} x_{2}\left|x_{1}\right|^{2\left(j_{1}+m_{1}\right)}\left|x_{2}\right|^{2\left(j_{2}+m_{2}\right)} \times \\
& \quad\left|1-x_{1}\right|^{2\left(j_{2}-j_{1}-j_{3}-1\right)}\left|1-x_{2}\right|^{2\left(j_{1}-j_{2}-j_{3}-1\right)}\left|x_{1}-x_{2}\right|^{2\left(j_{3}-j_{1}-j_{2}-1\right)} .
\end{aligned}
$$

The integral over $x$ in (B.23) ensures winding number conservation $m_{1}+m_{2}+m_{3}=0$. The function $F($ B.24) does not seem to be expressible in terms of elementary functions. The same two-point functions and three-point functions arise also in the coset $S L(2) / U(1)$ when we look at correlation functions preserving the winding number; in the coset, additional correlation functions are non-vanishing as well.

A special case that plays a role in $\S 5$ is

$$
\left\langle\tilde{\Phi}_{-j_{1}-1 ; j_{1}+1, j_{1}+1} \tilde{\Phi}_{-j_{2}-1 ; j_{2}+1, j_{2}+1} \tilde{\Phi}_{-j_{1}-j_{2}-2 ;-j_{1}-j_{2}-2,-j_{1}-j_{2}-2}\right\rangle
$$

As we argued in section 5 this correlator computes the residue of the pole in the correlator of non-normalizable operators $\tilde{\Phi}_{\tilde{j}_{i} ; j_{i}+1, j_{i}+1}$ as $\tilde{j}_{i}$ approaches $j_{i}$. This residue is computed in [7], and in the normalization (B.17) it takes the following form :

$$
\left\langle\tilde{\Phi}_{-j_{1}-1 ; j_{1}+1, j_{1}+1} \tilde{\Phi}_{-j_{2}-1 ; j_{2}+1, j_{2}+1} \tilde{\Phi}_{-j_{3}-1 ;-j_{3}-1,-j_{3}-1}\right\rangle=\frac{k \pi}{2} \sqrt{\frac{\gamma\left(1-\frac{2 j_{1}+1}{k}\right) \gamma\left(1-\frac{2 j_{2}+1}{k}\right)}{\gamma\left(\frac{1}{k}\right) \gamma\left(1-\frac{2 j_{3}+1}{k}\right)}}
$$

where $j_{3}=j_{1}+j_{2}+1$. 


\section{Appendix C. Normalization of two-point functions in Liouville and $S L(2)$ back- grounds of string theory}

In string theory in flat space-time $\mathbb{R}^{d-1,1}$, it is well-known that the two-point function of physical, on shell, operators on the sphere (as well as the zero and one-point functions, which we will not discuss here) vanishes. From the worldsheet point of view this is natural since the CFT two-point function is finite2 2 , but one has to divide by the volume of the conformal Killing group ( $\mathrm{CKG}$ ) of the sphere with two punctures, which is infinite. Equivalently, the two-point function vanishes since it does not saturate the zero modes of the reparametrization ghosts $c, \bar{c}$ on the sphere. In space-time, this is natural as well, since the two-point function corresponds to the inverse propagator $p^{2}+m^{2}$, which indeed vanishes on-shell.

In backgrounds that involve $S L(2)$, such as Liouville theory, $A d S_{3}, S L(2) / U(1)$, or the Nappi-Witten spacetime [82], it is similarly well-known that the two-point function (as well as the zero and one-point function) does not vanish 28 . From the worldsheet point of view this is due to the fact that while in string theory one still needs to divide by the volume of the conformal Killing group of the sphere with two punctures, the CFT correlator is typically infinite, due to a diverging integral over bosonic zero modes in the CFT. This infinity precisely cancels the volume of the conformal Killing group, and leaves behind a finite answer.

From the space-time point of view this is natural as well. Correlation functions of nonnormalizable operators in such backgrounds correspond to off-shell Green's functions in a dual "boundary theory". In Liouville theory and $S L(2) / U(1)$ the dual theory is in general a LST [3], and in particular low dimensional examples it can be described alternatively by a large $N$ matrix model. In $A d S_{3}$, the dual theory is a two dimensional CFT [50]. In the Nappi-Witten model, the dual is not known but is expected to exist [83]. In all these cases, the two-point function is expected to be non-zero in general.

To compute the finite two-point function in string theory in the backgrounds mentioned above, one has to evaluate the ratio of infinite volumes of the bosonic zero modes in the CFT and the conformal Killing group of the sphere with two punctures. Naively one

27 It is typically proportional to the volume of space-time, which is infinite, but, like in field theory (in momentum space), one is interested in the contribution to the correlation function that is proportional to the volume, i.e. preserves momentum.

28 This is also true for backgrounds involving other asymptotically $A d S$ spaces. 
might expect that this just gives a constant, independent of the quantum numbers of the operators whose two-point function is being computed, but this is known to be incorrect. The purpose of this appendix is to compute the two-point function in string theory for $S L(2) / U(1)$. We start with a discussion of the more familiar Liouville case, and then move on to $S L(2)$. On general grounds, one expects that the ratio of determinants in question should be the same in Liouville theory, $S L(2), S L(2) / U(1)$, and other related theories, since it has to do with the same divergence in the underlying $S L(2) \mathrm{CFT}$. We will see that this is indeed the case.

We start with (bosonic) Liouville theory. We will use results from 84 but modify them to be consistent with our normalizations and conventions 29 in appendix A. The central charge of the Liouville theory is related to the linear dilaton slope $Q$ as follows:

$$
c=1+3 Q^{2}
$$

The Lagrangian contains an interaction term

$$
\mathcal{L}_{\text {int }}=\mu e^{-\sqrt{2} b \phi},
$$

where $b$ is defined by the relation $Q / \sqrt{2}=b+b^{-1}$. The operators of interest are

$$
V_{\alpha}=e^{-\sqrt{2} \alpha \phi}
$$

Their worldsheet scaling dimensions are (A.11)

$$
\Delta(\alpha)=\alpha\left(\frac{Q}{\sqrt{2}}-\alpha\right)
$$

Note that the Liouville term in the Lagrangian (C.2) is $\mu V_{b}$; according to (C.4) it is marginal.

The two-point function of the operators $V_{\alpha}$ is given by

$$
\left\langle V_{\alpha_{1}}(z) V_{\alpha_{2}}(0)\right\rangle=\frac{\delta\left(\alpha_{1}-\alpha_{2}\right) D\left(\alpha_{1}\right)}{|z|^{4 \Delta\left(\alpha_{1}\right)}}
$$

where

$$
D(\alpha) \equiv\left(\pi \mu \gamma\left(b^{2}\right)\right)^{\frac{1}{b}\left(\frac{Q}{\sqrt{2}}-2 \alpha\right)} \frac{\gamma\left(2 b \alpha-b^{2}\right)}{b^{2} \gamma\left(2-\frac{2 \alpha}{b}+\frac{1}{b^{2}}\right)}
$$

29 Because of different choices for $\alpha^{\prime}$ and for the sign of $\phi$ in [84, this involves multiplying $Q$ in that paper by $1 / \sqrt{2}$ and multiplying $\phi$ by $(-1 / \sqrt{2})$. 
with $\gamma(x)$ defined in (B.8). As mentioned above, this CFT result is divergent when $\alpha_{1}=\alpha_{2}$. The divergence arises from the integration over the bosonic zero mode of $\phi$ in the CFT, and is cancelled in string theory by the volume of the corresponding conformal Killing group. The question we would like to address is what is the finite result obtained by taking into account this ratio of infinite factors.

A simple trick that allows one to resolve this issue is to differentiate the two-point function (C.5) with respect to the cosmological constant $\mu$. This brings down from the action the operator $V_{b}$ (with a minus sign). In the CFT this operator is integrated over the worldsheet, and the divergent factor $\delta\left(\alpha_{1}-\alpha_{2}\right)$ arises from the integral. In string theory, it is clear that the right prescription is to drop the integral over $V_{b}$ and replace it by $c \bar{c} V_{b}$. This eliminates both of the infinities mentioned above. The (unintegrated) Liouville threepoint function $\left\langle V_{b} V_{\alpha} V_{\alpha}\right\rangle$ is finite, and all the $c, \bar{c}$ zero modes on the sphere are soaked by the vertex operators, or equivalently, the conformal Killing group of the sphere with three punctures is trivial. Thus, we conclude that the two-point function $\left\langle V_{\alpha} V_{\alpha}\right\rangle$ in string theory can be obtained by integrating the relation

$$
\frac{\partial}{\partial \mu}\left\langle V_{\alpha} V_{\alpha}\right\rangle_{\text {string }}=-\left\langle V_{b} V_{\alpha} V_{\alpha}\right\rangle
$$

The three-point function on the right-hand side of (C.7) is a special case of those calculated in 85,86. Using the results of these papers and integrating (C.7), one finds that

$$
\left\langle V_{\alpha} V_{\alpha}\right\rangle_{\text {string }}=\frac{1}{\pi}\left(\frac{Q}{\sqrt{2}}-2 \alpha\right) D(\alpha) \text {. }
$$

Comparing to (C.5) we see that the ratio of infinite volumes produces in this case the finite factor $(Q / \sqrt{2}-2 \alpha) / \pi$, which depends on the particular operators whose correlation function is being computed.

We next move on to the case of $S L(2)$. This case was already analyzed by slightly different methods in [67, with results that agree with the results that we will find below 30 . The two-point function in the $S L(2)$ CFT is given in (B.15). Restoring the $z$-dependence, we can write it as

$$
\left\langle\Phi_{j_{1}}^{(s l)}\left(x_{1} ; z_{1}\right) \Phi_{j_{2}}^{(s l)}\left(x_{2} ; z_{2}\right)\right\rangle=\frac{\delta\left(j_{1}-j_{2}\right) D\left(j_{1}\right)}{\left|x_{12}\right|^{4\left(j_{1}+1\right)}\left|z_{12}\right|^{4 \Delta\left(j_{1}\right)}},
$$

30 The results stated in 67 include only the $j$-dependent factors in the two-point functions and not the additional $j$-independent factors. Reinstating these factors the result precisely agrees with our result below. 
where $\Delta(j)$ is given by $(\mathbb{B . 1 4})$ and

$$
D(j)=\frac{k}{\pi}\left[\frac{1}{k \pi} \gamma\left(\frac{1}{k}\right)\right]^{2 j+1} \gamma\left(1-\frac{2 j+1}{k}\right) .
$$

Again, in order to compute the corresponding correlation function in string theory we need to resolve the ratio of infinite factors coming from the $\delta\left(j_{1}-j_{2}\right)$, and from the volume of the CKG. To do that we will use a result from [58], where it is proven that (in the notations of this paper) the following identity should hold in string theory (see equation $(3.28)$ in [58]):

$$
\left\langle I \Phi_{j}^{(s l)}\left(x_{1}, \bar{x}_{1}\right) \Phi_{j}^{(s l)}\left(x_{2}, \bar{x}_{2}\right)\right\rangle=\frac{2 j+1}{k}\left\langle\Phi_{j}^{(s l)}\left(x_{1}, \bar{x}_{1}\right) \Phi_{j}^{(s l)}\left(x_{2}, \bar{x}_{2}\right)\right\rangle,
$$

where

$$
I=\frac{1}{k^{2}} \int d^{2} z J(x ; z) \bar{J}(\bar{x} ; \bar{z}) \Phi_{0}^{(s l)}(x, \bar{x} ; z, \bar{z}) .
$$

See e.g. [58] for the definition and properties of the $S L(2, \mathbb{R})$ current $J(x ; z)$. Equation (C.11) is a special case of a more general relation that is proven in [58]. Just like for the Liouville case, in order to make sense of (C.11) in string theory, we drop the integral in the definition of $I$ (C.12), compute the left-hand side of (C.11) (which involves no divergences) and take it to be the definition of the right-hand side. Using the results of appendix B and the Ward identities of $S L(2)$ currents summarized in [58, one finds that (suppressing the dependence on $x$ and $z$ )

$$
\left\langle I \Phi_{j}^{(s l)} \Phi_{j}^{(s l)}\right\rangle=\frac{1}{2 \pi^{2}}\left(\frac{2 j+1}{k}\right)^{2} D(j) .
$$

Substituting into (C.11) we conclude that the string theory two-point function is given by (again suppressing the dependence on $x$ and $z$ )

$$
\left\langle\Phi_{j}^{(s l)} \Phi_{j}^{(s l)}\right\rangle_{\text {string }}=\frac{1}{2 \pi^{2}} \frac{2 j+1}{k} D(j) .
$$

The $S L(2) / U(1)$ result follows immediately from this. Comparing to (C.9) we see that the string two-point function is corrected relative to the coefficient of $\delta\left(j_{1}-j_{2}\right)$ in the CFT two-point function by the factor $\frac{1}{2 \pi^{2}} \frac{2 j+1}{k}$. To compare to the Liouville result (C.8) we need to take into account the relation between the Liouville momentum $\alpha$ and $j, \alpha=-Q j / \sqrt{2}$. Thus, $Q / \sqrt{2}-2 \alpha=Q(1+2 j) / \sqrt{2}$. This has to be multiplied further by $Q / \sqrt{2}$ to account for the difference between $\delta\left(\alpha_{1}-\alpha_{2}\right)$ in (C.5), and $\delta\left(j_{1}-j_{2}\right)$ in (C.9). Thus, the Liouville answer is in $S L(2)$ variables $31 \frac{1}{2 \pi} Q^{2}(2 j+1)=\frac{1}{\pi} \frac{2 j+1}{k}$. The $S L(2)$ answer (C.14) has the same dependence on $j$ and $k$; it differs from the Liouville result by the factor $1 / 2 \pi$. We do not know the origin of this minor discrepancy.

31 Recall that in our conventions $Q^{2}=2 / k$ 4.7). 


\section{Appendix D. Simplification of the formula for $t_{n_{1}, n_{2}, n_{3}, n_{4}}$}

In $\S 6.1$ we found the formula

$$
t_{n_{1}, n_{2}, n_{3}, n_{4}}=\frac{1}{r_{0}^{2}} \sum_{l>m=0}^{k-1} \frac{e^{-\frac{\pi i}{k}(l+m) \sum n_{i}}}{\sin ^{2}\left[\frac{\pi(l-m)}{k}\right]} \prod_{i=1}^{4} \sin \left[\frac{\pi(l-m)}{k} n_{i}\right]
$$

which we would like to simplify here. Let us introduce a new summation variable $p=l-m$. In terms of this variable, the sum in (D.1) can be rewritten as

$$
\sum_{l>m=0}^{k-1}=\sum_{p=0}^{k-1} \sum_{m=0}^{k-1-p}
$$

where we added a $p=0$ term without changing the expression, since the summand vanishes for $p=0$. Using the relation

$$
\sin n \alpha=\sin \alpha \sum_{l=0}^{n-1} e^{i(n-2 l-1) \alpha}
$$

one can further simplify (D.1) to the form

$$
t_{n_{1}, n_{2}, n_{3}, n_{4}}=\frac{1}{r_{0}^{2}} \sum_{p=0}^{k-1} \sum_{m=0}^{k-1-p} e^{-\frac{2 \pi i m}{k} \sum n_{i}} \sum_{l_{i}=0}^{n_{i}-1} e^{-\frac{2 \pi i p}{k}\left(\sum l_{i}+2\right)} \sin ^{2}\left(\frac{2 \pi p}{k}\right) .
$$

The behavior of the sum over $m$ in the expression above depends on the value of $\sum n_{i}$. Let us first consider the case $\sum n_{i} \notin k \mathbb{Z}$. Then, one can easily do the sum over $m$ and find

$$
\begin{aligned}
t_{n_{1}, n_{2}, n_{3}, n_{4}}= & \frac{k}{4 r_{0}^{2}}\left(e^{-\frac{2 \pi i}{k} \sum n_{i}}-1\right)^{-1} \times \\
& {\left[\sum_{l_{i}=0}^{n_{i}-1}\left(\delta \sum n_{i}-\sum l_{i}-3, k \mathbb{Z}+\delta \sum n_{i}-\sum l_{i}-1, k \mathbb{Z}-2 \delta \sum n_{i}-\sum l_{i}-2, k \mathbb{Z}\right)-\right.} \\
& \sum_{l_{i}=0}^{n_{i}-1}\left(\delta \sum l_{i}+3, k \mathbb{Z}\right. \\
& \left.\left.+\delta \sum l_{i}+1, k \mathbb{Z}-2 \delta \sum l_{i}+2, k \mathbb{Z}\right)\right] .
\end{aligned}
$$

It is easy to see that this expression is actually zero. Indeed, by a change of variables

$$
l_{i} \rightarrow \tilde{l}_{i}=n_{i}-l_{i}-1
$$

one can show that the third line of (D.5) exactly cancels the second line. This is precisely what we expect, since the theory at the specific point of moduli space we are at has a $\mathbb{Z}_{k}$ 
symmetry under which the $\mathcal{O}_{n}^{+}$have charge $n$, and this symmetry should not be broken by the $F^{4}$ vertex.

Now we turn to the case $\sum n_{i} \in k \mathbb{Z}$. Define $N$ via $\sum n_{i}=k N$. The coupling in this case takes the form

$$
t_{n_{1}, n_{2}, n_{3}, n_{4}}=\frac{1}{r_{0}^{2}} \sum_{p=0}^{k-1}(k-p) \frac{(-1)^{N p}}{\sin ^{2} \frac{p \pi}{k}} \prod_{i=1}^{4} \sin \frac{\pi p}{k} n_{i} \equiv \sum_{p=0}^{k-1}(k-p) f(p) .
$$

The function $f(p)$ introduced in (D.7) has the following properties :

$$
f(p)=f(-p)=f(p+k) ; \quad f(0)=0 .
$$

Using these properties one can easily show that

$$
\sum_{p=0}^{k-1}(k-p) f(p)=\frac{k}{2} \sum_{p=0}^{k-1} f(p)
$$

so we conclude that

$$
t_{n_{1}, n_{2}, n_{3}, n_{4}}=\frac{k}{2 r_{0}^{2}} \sum_{p=0}^{k-1} \frac{(-1)^{N p}}{\sin ^{2} \frac{\pi p}{k}} \prod_{i=1}^{4} \sin \frac{\pi p}{k} n_{i}
$$

Without loss of generality we will assume that $n_{1} \leq n_{2} \leq n_{3} \leq n_{4}$. We can use the following identity to simplify (D.10) :

$$
\sum_{n=0}^{\min \left(r, r^{\prime}\right)-1} \sin \frac{\pi p}{k} \sin \frac{\pi p}{k}\left(r+r^{\prime}-2 n-1\right)=\sin \left(\frac{\pi p}{k} r\right) \sin \left(\frac{\pi p}{k} r^{\prime}\right) .
$$

Then, (D.10) takes the form

$$
\begin{aligned}
t_{n_{1}, n_{2}, n_{3}, n_{4}}=-\frac{k}{4 r_{0}^{2}} \sum_{p=0}^{k-1}(-1)^{N p} \sum_{l_{1}=0}^{n_{1}-1} \sum_{l_{3}=0}^{n_{3}-1}\left[\cos \frac{\pi p}{k}\left(N k-2\left(l_{1}+l_{3}\right)-2\right)-\right. \\
\left.\cos \frac{\pi p}{k}\left(n_{1}+n_{2}-n_{3}-n_{4}-2\left(l_{1}-l_{3}\right)\right)\right] .
\end{aligned}
$$

Simplifying, one arrives at the following expression for the coupling

$$
t_{n_{1}, n_{2}, n_{3}, n_{4}}=-\frac{k^{2}}{4 r_{0}^{2}} \sum_{l_{1}=0}^{n_{1}-1} \sum_{l_{3}=0}^{n_{3}-1}\left[\delta_{l_{1}+l_{3}+1, k \mathbb{Z}}-\delta_{l_{1}+l_{3}+n_{4}+1, k \mathbb{Z}}\right] .
$$


Let us analyze this result. Since the $n_{i}$ lie between 1 and $k-1$, the sum of the $n_{i}$ 's is

$$
4 \leq \sum_{i=1}^{4} n_{i} \leq 4 k-4
$$

or equivalently $1 \leq N \leq 3$ (recall that $N=\frac{1}{k} \sum_{i} n_{i}$ must be integer to get a non-vanishing coupling), which means that we have three cases to consider. Let us consider them in turn.

(1) For $N=1$ one can see that neither of the two delta functions in (D.13) is saturated and hence the coupling in this case is zero.

(2) For $N=2$ we see from the ordering of the $n_{i}$ that

$$
\begin{aligned}
& n_{1}+n_{3} \leq n_{2}+n_{4} \Rightarrow n_{1}+n_{3} \leq k, \\
& n_{1}+n_{2} \leq n_{3}+n_{4} \Rightarrow n_{1}+n_{2} \leq k
\end{aligned}
$$

which means that the first delta function in (D.13) is never saturated, while the second gives the following value for the coupling

$$
t_{n_{1}, n_{2}, n_{3}, n_{4}}=\frac{k^{2}}{4 r_{0}^{2}} \min \left(n_{1}, k-n_{4}\right) .
$$

(3) For $N=3$ we conclude using $n_{1} \leq n_{2} \leq n_{3} \leq n_{4} \leq k-1$ that

$$
k+2 \leq n_{1}+n_{3} \leq\left[\frac{3 k}{2}\right] ; \quad n_{i}+n_{j} \geq k+2, \forall i, j
$$

where $[3 k / 2]$ is the integer part of $3 k / 2$. From this we conclude that the contribution from the first delta function is

$$
\sum_{l_{1}=0}^{n_{1}-1} \sum_{l_{3}=0}^{n_{3}-1} \delta_{l_{1}+l_{3}+1, k \mathbb{Z}}=\min \left(n_{1}, n_{1}+n_{3}-k\right)=n_{1}+n_{3}-k
$$

while the second delta function contributes

$-\sum_{l_{1}=0}^{n_{1}-1} \sum_{l_{3}=0}^{n_{3}-1} \delta_{l_{1}+l_{3}+n_{4}+1, k \mathbb{Z}}=-\left(\min \left(n_{1}, k-n_{4}\right)+\min \left(n_{1}, k-n_{2}\right)\right)=-\left(2 k-n_{2}-n_{4}\right)$.

We see that in this case the coupling is again vanishing.

To summarize, using the symmetry of $t$ in its four indices, we found that

$$
t_{n_{1}, n_{2}, n_{3}, n_{4}}=\frac{k^{2}}{4 r_{0}^{2}} \min \left(n_{i}, k-n_{i}\right)
$$


if $\sum n_{i}=2 k$, and it vanishes otherwise.

It is interesting to note that $(\mathrm{D} .20)$ can be written in terms of the fusion coefficients of the $S U(2)$ WZW theory of level $k-2$,

$$
t_{n_{1}, n_{2}, n_{3}, n_{4}}=\frac{k^{2}}{4 r_{0}^{2}} \sum_{l=0}^{k-1} N_{n_{1}-1, n_{2}-1}^{l} N_{n_{3}-1, n_{4}-1}^{l} \quad \text { for } \sum n_{i}=2 k
$$

where (see for example [87])

$$
N_{n_{2}-1, n_{3}-1}^{n_{1}-1}=\frac{2}{k} \sum_{p=1}^{k-1} \frac{1}{\sin \frac{\pi p}{k}} \sin \frac{\pi p n_{1}}{k} \sin \frac{\pi p n_{2}}{k} \sin \frac{\pi p n_{3}}{k}
$$

are the fusion coefficients of $S U(2)_{k-2}$,

$$
N_{l_{1}, l_{2}}^{l_{3}}= \begin{cases}1 & \text { if } \quad\left|l_{1}-l_{2}\right| \leq l_{3} \leq \min \left(l_{1}+l_{2}, 2(k-2)-l_{1}-l_{2}\right) \\ 0 & \text { otherwise }\end{cases}
$$

The indices $l_{i}$ are related to the $S U(2)$ spins $j_{i}$, which label the primaries of $S U(2)_{k-2}$, as follows: $l_{i}=2 j_{i}$. A useful identity for verifying (D.21) is:

$$
\sum_{l=0}^{k-1} \sin \frac{\pi m l}{k} \sin \frac{\pi n l}{k}=\frac{k}{2}\left(\delta_{m-n, 2 k \mathbb{Z}}-\delta_{m+n, 2 k \mathbb{Z}}\right)
$$




\section{References}

[1] O. Aharony, "A brief review of 'little string theories'," Class. Quant. Grav. 17, 929 (2000) arXiv:hep-th/9911147.

[2] D. Kutasov, "Introduction to little string theory," prepared for ICTP Spring School on Superstrings and Related Matters, Trieste, Italy, 2-10 April 2001.

[3] O. Aharony, M. Berkooz, D. Kutasov and N. Seiberg, "Linear dilatons, NS5-branes and holography," JHEP 9810, 004 (1998) arXiv:hep-th/9808149.

[4] O. Aharony, M. Berkooz, S. Kachru, N. Seiberg and E. Silverstein, "Matrix description of interacting theories in six dimensions," Adv. Theor. Math. Phys. 1, 148 (1998) arXiv:hep-th/9707079; E. Witten, "On the conformal field theory of the Higgs branch," JHEP 9707, 003 (1997) arXiv:hep-th/9707093.

[5] D. Kutasov and D. A. Sahakyan, "Comments on the thermodynamics of little string theory," JHEP 0102, 021 (2001) arXiv:hep-th/0012258.

[6] A. Giveon and D. Kutasov, "Little string theory in a double scaling limit," JHEP 9910, 034 (1999) arXiv:hep-th/9909110.

[7] A. Giveon and D. Kutasov, "Comments on double scaled little string theory," JHEP 0001, 023 (2000) arXiv:hep-th/9911039.

[8] M. Bershadsky, S. Cecotti, H. Ooguri and C. Vafa, "Kodaira-Spencer theory of gravity and exact results for quantum string amplitudes," Commun. Math. Phys. 165, 311 (1994) arXiv:hep-th/9309140.

[9] N. Berkovits and C. Vafa, "N $=4$ topological strings," Nucl. Phys. B 433, 123 (1995) arXiv:hep-th/9407190.

[10] A. Giveon and M. Rocek, "On the BRST operator structure of the $N=2$ string," Nucl. Phys. B 400, 145 (1993) arXiv:hep-th/9302049.

[11] R. Gopakumar and C. Vafa, "Topological gravity as large $N$ topological gauge theory," Adv. Theor. Math. Phys. 2, 413 (1998) arXiv:hep-th/9802016].

[12] R. Gopakumar and C. Vafa, "On the gauge theory/geometry correspondence," Adv. Theor. Math. Phys. 3, 1415 (1999) arXiv:hep-th/9811131.

[13] H. Ooguri and C. Vafa, "Worldsheet derivation of a large $N$ duality," Nucl. Phys. B 641, 3 (2002) arXiv:hep-th/0205297.

[14] C. Vafa, "Superstrings and topological strings at large N," J. Math. Phys. 42, 2798 (2001) arXiv:hep-th/0008142.

[15] N. Berkovits, H. Ooguri and C. Vafa, "On the worldsheet derivation of large $N$ dualities for the superstring," arXiv:hep-th/0310118.

[16] C. G. Callan, J. A. Harvey and A. Strominger, "Supersymmetric string solitons," arXiv:hep-th/9112030.

[17] J. McGreevy and H. Verlinde, "Strings from tachyons: the $c=1$ matrix reloaded," arXiv:hep-th/0304224. 
[18] E. J. Martinec, "The annular report on non-critical string theory," arXiv:hepth/0305148.

[19] I. R. Klebanov, J. Maldacena and N. Seiberg, "D-brane decay in two-dimensional string theory," JHEP 0307, 045 (2003) arXiv:hep-th/0305159.

[20] J. McGreevy, J. Teschner and H. Verlinde, "Classical and quantum D-branes in 2D string theory," arXiv:hep-th/0305194.

[21] S. Y. Alexandrov, V. A. Kazakov and D. Kutasov, "Non-perturbative effects in matrix models and D-branes," JHEP 0309, 057 (2003) arXiv:hep-th/0306177.

[22] A. Sen, "Open-closed duality: Lessons from matrix model," arXiv:hep-th/0308068.

[23] T. Takayanagi and N. Toumbas, "A matrix model dual of type 0B string theory in two dimensions," JHEP 0307, 064 (2003) arXiv:hep-th/0307083.

[24] M. R. Douglas, I. R. Klebanov, D. Kutasov, J. Maldacena, E. Martinec and N. Seiberg, "A new hat for the $c=1$ matrix model," arXiv:hep-th/0307195.

[25] I. R. Klebanov, J. Maldacena and N. Seiberg, "Unitary and complex matrix models as 1-d type 0 strings," arXiv:hep-th/0309168.

[26] A. Giveon, D. Kutasov and O. Pelc, "Holography for non-critical superstrings," JHEP 9910, 035 (1999) arXiv:hep-th/9907178.

[27] M. Dine and N. Seiberg, "Comments on higher derivative operators in some SUSY field theories," Phys. Lett. B 409, 239 (1997) arXiv:hep-th/9705057.

[28] M. Henningson, "Extended superspace, higher derivatives and $S L(2, Z)$ duality," Nucl. Phys. B 458, 445 (1996) arXiv:hep-th/9507135.

[29] B. de Wit, M. T. Grisaru and M. Rocek, "Nonholomorphic corrections to the oneloop $N=2$ Super Yang-Mills action," Phys. Lett. B 374, 297 (1996) arXiv:hepth/9601115.

[30] U. Lindstrom, F. Gonzalez-Rey, M. Rocek and R. von Unge, "On $N=2$ low energy effective actions," Phys. Lett. B 388, 581 (1996) arXiv:hep-th/9607089].

[31] I. L. Buchbinder and E. A. Ivanov, "Complete $N=4$ structure of low-energy effective action in $N=4$ super Yang-Mills theories," Phys. Lett. B 524, 208 (2002) arXiv:hepth/0111062].

[32] A. T. Banin, I. L. Buchbinder and N. G. Pletnev, "One-loop effective action for $N=$ 4 SYM theory in the hypermultiplet sector: Leading low-energy approximation and beyond," arXiv:hep-th/0304046.

[33] D. A. Lowe and R. von Unge, "Constraints on higher derivative operators in maximally supersymmetric gauge theory," JHEP 9811, 014 (1998) arXiv:hep-th/9811017.

[34] F. Gonzalez-Rey, B. Kulik, I. Y. Park and M. Rocek, "Self-dual effective action of $N=4$ super-Yang-Mills," Nucl. Phys. B 544, 218 (1999) arXiv:hep-th/9810152.

[35] E. I. Buchbinder, I. L. Buchbinder and S. M. Kuzenko, "Non-holomorphic effective potential in $N=4 S U(n)$ SYM," Phys. Lett. B 446, 216 (1999) arXiv:hep-th/9810239. 
[36] J. R. Ellis, P. Jetzer and L. Mizrachi, "One loop string corrections to the effective field theory," Nucl. Phys. B 303, 1 (1988).

[37] M. Abe, H. Kubota and N. Sakai, "Loop corrections to the $E_{8} \times E_{8}$ heterotic string effective Lagrangian," Nucl. Phys. B 306, 405 (1988); Phys. Lett. B 200, 461 (1988) [Addendum-ibid. B 203, 474 (1988)].

[38] W. Lerche, "Elliptic index and superstring effective actions," Nucl. Phys. B 308, 102 (1988).

[39] J. Polchinski, "String Theory. Vol. 2: Superstring theory and beyond," Cambridge University Press, 1998.

[40] E. Kiritsis, "Duality and instantons in string theory," arXiv:hep-th/9906018.

[41] C. Bachas, C. Fabre, E. Kiritsis, N. A. Obers and P. Vanhove, "Heterotic/type-I duality and D-brane instantons," Nucl. Phys. B 509, 33 (1998) arXiv:hep-th/9707126.

[42] S. Stieberger and T. R. Taylor, "Non-Abelian Born-Infeld action and type I - heterotic duality. II: Nonrenormalization theorems," Nucl. Phys. B 648, 3 (2003) arXiv:hepth/0209064.

[43] I. Antoniadis, E. Gava, K. S. Narain and T. R. Taylor, "Topological amplitudes in string theory," Nucl. Phys. B 413, 162 (1994) arXiv:hep-th/9307158; "N= 2 type II heterotic duality and higher derivative F terms," Nucl. Phys. B 455, 109 (1995) arXiv:hep-th/9507115.

[44] A. Dabholkar and J. A. Harvey, "Nonrenormalization of the superstring tension," Phys. Rev. Lett. 63, 478 (1989).

[45] C. M. Hull and P. K. Townsend, "Unity of superstring dualities," Nucl. Phys. B 438, 109 (1995) arXiv:hep-th/9410167.

[46] E. Kiritsis, N. A. Obers and B. Pioline, "Heterotic/type II triality and instantons on K3," JHEP 0001, 029 (2000) arXiv:hep-th/0001083.

[47] H. Ooguri and C. Vafa, "Two-Dimensional black hole and singularities of CY manifolds," Nucl. Phys. B 463, 55 (1996) arXiv:hep-th/9511164.

[48] D. Kutasov, "Orbifolds and solitons," Phys. Lett. B 383, 48 (1996) arXiv:hepth/9512145].

[49] J. A. Harvey, D. Kutasov, E. J. Martinec and G. Moore, "Localized tachyons and RG flows," arXiv:hep-th/0111154.

[50] J. M. Maldacena, "The large $N$ limit of superconformal field theories and supergravity," Adv. Theor. Math. Phys. 2, 231 (1998) [Int. J. Theor. Phys. 38, 1113 (1999)] arXiv:hep-th/9711200; S. S. Gubser, I. R. Klebanov and A. M. Polyakov, "Gauge theory correlators from non-critical string theory," Phys. Lett. B 428, 105 (1998) arXiv:hep-th/9802109; E. Witten, "Anti-de Sitter space and holography," Adv. Theor. Math. Phys. 2, 253 (1998) arXiv:hep-th/9802150; O. Aharony, S. S. Gubser, J. M. Maldacena, H. Ooguri and Y. Oz, "Large $N$ field theories, string theory and gravity," Phys. Rept. 323, 183 (2000) arXiv:hep-th/9905111. 
[51] V. Balasubramanian, M. Berkooz, A. Naqvi and M. J. Strassler, "Giant gravitons in conformal field theory," JHEP 0204, 034 (2002) [arXiv:hep-th/0107119].

[52] S. Corley, A. Jevicki and S. Ramgoolam, "Exact correlators of giant gravitons from dual $N=4$ SYM theory," Adv. Theor. Math. Phys. 5, 809 (2002) arXiv:hep th/0111222]; S. Corley and S. Ramgoolam, "Finite factorization equations and sum rules for BPS correlators in $N=4$ SYM theory," Nucl. Phys. B 641, 131 (2002) arXiv:hep-th/0205221.

[53] O. Aharony, Y. E. Antebi, M. Berkooz and R. Fishman, "Holey sheets': Pfaffians and subdeterminants as D-brane operators in large $N$ gauge theories," JHEP 0212, 069 (2002) arXiv:hep-th/0211152.

[54] O. Aharony, A. Giveon and D. Kutasov, to appear.

[55] D. Kutasov, "Geometry On The Space Of Conformal Field Theories And Contact Terms," Phys. Lett. B 220, 153 (1989).

[56] V. Balasubramanian, P. Kraus and A. E. Lawrence, "Bulk vs. boundary dynamics in anti-de Sitter spacetime," Phys. Rev. D 59, 046003 (1999) arXiv:hep-th/9805171.

[57] K. Hori and A. Kapustin, "Duality of the fermionic $2 \mathrm{~d}$ black hole and $N=2$ Liouville theory as mirror symmetry," JHEP 0108, 045 (2001) arXiv:hep-th/0104202.

[58] A. Giveon and D. Kutasov, "Notes on $A d S_{3}$," Nucl. Phys. B 621, 303 (2002) arXiv:hep-th/0106004.

[59] D. Tong, "Mirror mirror on the wall: on two-dimensional black holes and Liouville theory," JHEP 0304, 031 (2003) arXiv:hep-th/0303151.

[60] E. Gava, K. S. Narain and M. H. Sarmadi, "Little string theories in heterotic backgrounds," Nucl. Phys. B 626, 3 (2002) [arXiv:hep-th/0112200].

[61] Z. A. Qiu, "Nonlocal current algebra and $N=2$ superconformal field theory in twodimensions," Phys. Lett. B 188, 207 (1987).

[62] V. A. Fateev and A. B. Zamolodchikov, "Parafermionic currents in the twodimensional conformal quantum field theory and selfdual critical points in $Z_{N}$ invariant statistical systems," Sov. Phys. JETP 62, 215 (1985) [Zh. Eksp. Teor. Fiz. 89, $380(1985)]$.

[63] J. M. Maldacena and H. Ooguri, "Strings in $A d S_{3}$ and $S L(2, R)$ WZW model. I," J. Math. Phys. 42, 2929 (2001) arXiv:hep-th/0001053.

[64] A. Parnachev and D. A. Sahakyan, "Some remarks on D-branes in $A d S_{3}$," JHEP 0110, 022 (2001) arXiv:hep-th/0109150.

[65] A. Strominger, "Massless black holes and conifolds in string theory," Nucl. Phys. B 451, 96 (1995) arXiv:hep-th/9504090.

[66] P. Di Francesco and D. Kutasov, "World sheet and space-time physics in twodimensional (Super)string theory," Nucl. Phys. B 375, 119 (1992) arXiv:hepth/9109005]. 
[67] J. M. Maldacena and H. Ooguri, "Strings in $A d S_{3}$ and the $S L(2, R)$ WZW model. III: Correlation functions," Phys. Rev. D 65, 106006 (2002) arXiv:hep-th/0111180.

[68] N. Marcus, "A tour through $N=2$ strings," arXiv:hep-th/9211059; O. Lechtenfeld, "Mathematics and physics of $N=2$ strings," arXiv:hep-th/9912281.

[69] J. Bischoff and O. Lechtenfeld, "Path-integral quantization of the $(2,2)$ string," Int. J. Mod. Phys. A 12, 4933 (1997) arXiv:hep-th/9612218.

[70] K. Junemann, O. Lechtenfeld and A. D. Popov, "Non-local symmetries of the closed $N=2$ string," Nucl. Phys. B 548, 449 (1999) arXiv:hep-th/9901164.

[71] E. Witten, "Ground ring of two-dimensional string theory," Nucl. Phys. B 373, 187 (1992) arXiv:hep-th/9108004.

[72] A. B. Zamolodchikov and V. A. Fateev, "Operator algebra and correlation functions in the two-dimensional Wess-Zumino $S U(2) \times S U(2)$ chiral model," Sov. J. Nucl. Phys. 43, 657 (1986) [Yad. Fiz. 43, 1031 (1986)].

[73] R. Dijkgraaf, "Intersection theory, integrable hierarchies and topological field theory," arXiv:hep-th/9201003.

[74] H. Ooguri and C. Vafa, "All loop $\mathcal{N}=2$ string amplitudes," Nucl. Phys. B 451, 121 (1995) arXiv:hep-th/9505183.

[75] Y. K. Cheung, Y. Oz and Z. Yin, "Families of $N=2$ strings," arXiv:hep-th/0211147.

[76] D. Gluck, Y. Oz and T. Sakai, "The effective action and geometry of closed $N=2$ strings," JHEP 0307, 007 (2003) arXiv:hep-th/0304103.

[77] D. Gluck, Y. Oz and T. Sakai, "D-branes in $N=2$ strings," JHEP 0308, 055 (2003) arXiv:hep-th/0306112.

[78] C. Kounnas, M. Porrati and B. Rostand, "On $\mathcal{N}=4$ extended superliouville theory," Phys. Lett. B 258, 61 (1991).

[79] J. Teschner, "On structure constants and fusion rules in the $S L(2, C) / S U(2)$ WZNW model," Nucl. Phys. B 546, 390 (1999) arXiv:hep-th/9712256].

[80] J. Teschner, "Operator product expansion and factorization in the $H_{3}^{+}$WZNW model," Nucl. Phys. B 571, 555 (2000) arXiv:hep-th/9906215.

[81] V. A. Fateev, A. B. Zamolodchikov and Al. B. Zamolodchikov, unpublished.

[82] C. R. Nappi and E. Witten, "A closed, expanding universe in string theory," Phys. Lett. B 293, 309 (1992) arXiv:hep-th/9206078.

[83] S. Elitzur, A. Giveon, D. Kutasov and E. Rabinovici, "From big bang to big crunch and beyond," JHEP 0206, 017 (2002) arXiv:hep-th/0204189.

[84] V. Fateev, A. B. Zamolodchikov and A. B. Zamolodchikov, "Boundary Liouville field theory. I: Boundary state and boundary two-point function," arXiv:hep-th/0001012.

[85] H. Dorn and H. J. Otto, "Two and three point functions in Liouville theory," Nucl. Phys. B 429, 375 (1994) arXiv:hep-th/9403141]. 
[86] A. B. Zamolodchikov and A. B. Zamolodchikov, "Structure constants and conformal bootstrap in Liouville field theory," Nucl. Phys. B 477, 577 (1996) arXiv:hepth/9506136].

[87] K. Hori, A. Iqbal and C. Vafa, "D-branes and mirror symmetry," arXiv:hepth/0005247. 